\title{
CLATHRIN AND TOM1L1 REGULATE EPIDERMAL GROWTH FACTOR RECEPTOR SIGNALING AT THE PLASMA MEMBRANE
}

\author{
By Gurjeet Singh Judge \\ B.Sc., Ryerson University, 2013 \\ A thesis presented to Ryerson University in partial fulfillment of the requirements for the \\ degree of Master of Science in the Program of Molecular Science
}

Toronto, Ontario, Canada, 2015

(C) Gurjeet Singh Judge 2015 


\section{AUTHOR'S DECLARATION}

I hereby declare that I am the sole author of this thesis. This is a true copy of the thesis, including any required final revisions, as accepted by my examiners.

I authorize Ryerson University to lend this thesis to other institutions or individuals for the purpose of scholarly research.

I further authorize Ryerson University to reproduce this thesis by photocopying or by other means, in total or in part, at the request of other institutions or individuals for the purpose of scholarly research.

I understand that my thesis may be made electronically available to the public 
Clathrin and TOM1L1 regulate epidermal growth factor receptor signaling at the plasma membrane.

Master of Science (M.Sc.), 2015

Gurjeet Singh Judge

Molecular Science

Ryerson University

\begin{abstract}
:
Epidermal growth factor (EGF) receptor (EGFR) controls many aspects of cell physiology via the activation of intracellular signaling pathways. Aberrant EGFR signaling and overexpression of EGFR is a characteristic of various cancer types. Ligandbound EGFR elicits phosphorylation of Gab1, which activates phosphatidylinositol-3kinase (PI3K), which in turn leads to Akt phosphorylation and activation. Akt is a central regulator of cell survival and metabolism. Rapidly upon binding EGF, the receptor is recruited to clathrin-coated pits (CCPs), eventually leading to EGFR endocytosis. As such, receptor-proximal EGFR signaling occurs while the receptor is enriched with CCPs at the plasma membrane. We have recently found that clathrin, but not receptor endocytosis, is required for EGF-stimulated phosphorylation of Akt, yet how clathrin controls EGFR signaling at the plasma membrane remains poorly understood.

Clathrin interacts with and recruits numerous proteins to the plasma membrane. To examine how specific clathrin-interacting proteins may underlie the requirement for clathrin in EGFR signaling, I have examined the role of TOM1L1, which interacts with clathrin, EGFR and Src-family kinases (SFKs) through distinct domains. SiRNA gene silencing of TOM1L1 impairs EGF-stimulated Akt phosphorylation, as did expression of a dominant-interfering mutant of TOM1L1 deficient in clathrin binding. Using fluorescent labeling of clathrin, TOM1L1 and other signaling intermediates coupled to total internal reflection fluorescence microscopy; I found that CCPs are enriched in TOM1L1 and phosphorylated Gab1 upon EGF stimulation. These findings indicate that the clathrin-binding adaptor TOM1L1 regulates EGF-stimulated Akt phosphorylation, thus revealing a novel molecular mechanism of regulation of EGFR signaling at the plasma membrane.
\end{abstract}




\section{Acknowledgements:}

I would like to start by thanking my supervisor Dr. Antonescu as this would not have been possible without him. The amount of support and guidance provided by Dr. Antonescu during my course in the lab cannot be explained in words. As his first undergraduate student, having no experience in the lab, I was very anxious initially, but Dr. Antonescu was very diligent and patient with me immersing in the lab. His physical presence in the lab during my initial months helped me become quickly comfortable and confident in my newly developing skills. I could say without any hesitation that I learned from the best. Dr. Antonescu has played a huge part in my career path. He has shown me the value of research and stimulated my interest in pursuing graduate studies. He clearly makes it known that he is much a part of my research as I am. The strong sense of support that I derive from him pushes me to strive for excellence. I would also like to thank the members of my lab especially Camilo Garay, Ralph Christian Delos Santos and Leslie Bone for not overcasting their graduate shadow on my undergraduate soul. The support and friendship offered by them is beyond explanation. Stefanie Lucalrelli, Stephen Bautista and Tanveer Singh the lab would not have been the same without them. I would also like to sincerely thank Christopher Choy from the Botelho lab without who, the qRT-PCR would not have been possible. Finally I would like to thank the members of my supervisory committee Dr.Yuen and Dr. Botelho for their rigorous questioning and critical feedback during my committee meetings which has helped me become a better researcher and have an open perspective. 
Table of Contents:

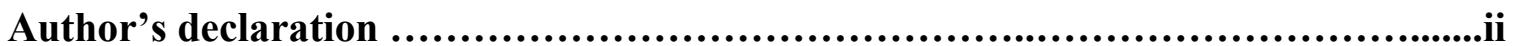

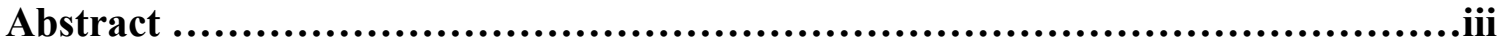

Acknowledgements.................................................................iv

List of figures........................................................................vii

Introduction............................................................................1

Receptor tyrosine kinases (RTKs).........................................2

Epidermal growth factor receptor (EGFR)..................................

EGFR stimulated activation of Akt..........................................5

Clathrin-mediated endocytosis (CME).....................................8

Formation of Clathrin Coated Pits (CCPs)...................................8

Regulation of EGFR by Clathrin.............................................12

Inhibition of Clathrin function................................................19

Previous approaches of CME inhibition...................................19

Rapamycin induced heterodimerization(aka Knocksideways silencing) as a

tool to achieve inhibition of specific proteins .....................................21

Src Family Kinases (SFKs) are required for EGF-stimulated Gab1 and Akt

phosphorylation......................................................................23

Clathrin: A protein scaffold..............................................24

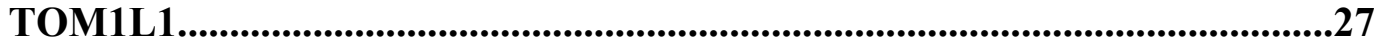

Overall Rationale..............................................................30

General Hypothesis.........................................................30

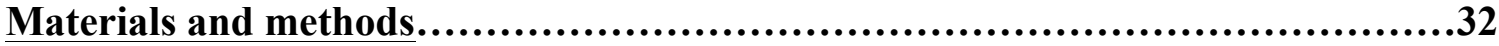

Antibodies and probes.........................................................32

Cell lines and Cell culture.....................................................32

Plasmids.......................................................................33

DNA plasmid preparation......................................................33

Transfections.....................................................................33

Cell lysis and immunoblotting................................................34

Microscopy and image analysis.............................................35

Immunofluorescence Staining.................................................36

Knocksideways Silencing...................................................37

Seamless Cloning (TOM1L1-GFP).........................................37

Agarose gel electrophoresis...................................................39

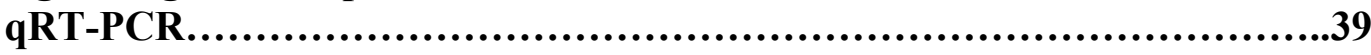

Results...............................................................................41

Expression of GFP-FKBP-CLCa and mitoTrap effectively relocalizes CHC

to the mitochondria..................................................................41 
Knocksideways silencing of clathrin light chain (CLC) reduces EGF stimulated Akt phosphorylation..................................................43

Expression of GFP-FKBP-CLCa and mitoTrap is of no effect on EGF stimulated Akt phosphorylation in the absence of rapamycin.......................45 p-Gab1 is recruited to Clathrin coated pits (CCPs) at the plasma membrane upon EGF stimulation..............................................................46

pGab1 is largely restricted to clathrin structures containing EGFR..........49 siRNA knockdown of TOM1L1 reduces EGF stimulated Akt phosphorylation....................................................................52 siRNA knockdown of TOM1L1 does not affect EGF stimulated MAPK and

EGFR phosphorylation...........................................................54

Generation of wt-TOM1L1-GFP..........................................56

TOM1L1 is constitutively detected within CCPs............................57

Expression of a clathrin binding-defective mutant of TOM1L1 affects basal and EGF-stimulated Akt phosphorylation.....................................59

Discussion..........................................................................64

Defining the role of Clathrin in EGF stimulated Akt phosohorylation using knocksideways silencing..........................................................64

CCPs as signaling microdomains at the plasma membrane.................67

TOM1L1 and Clathrin: Regulation of EGFR signalling.....................72

References...............................................................................80 


\section{List of Figures:}

Figure 1: Schematic of EGFR signaling.............................................

Figure 2: Formation of clathrin-coated pits at the plasma membrane $\ldots \ldots \ldots \ldots \ldots \ldots \ldots . . .1$

Figure 3: Silencing of CHC or Dyn2 results in similar inhibition of EGFR endocytosis yet distinct effects on EGF-stimulated Akt activation................................17

Figure 4: Treatment of RPE cells with the CHC-inhibitor Pitstop2 prevents EGFstimulated Gab1 and Akt phosphorylation but not EGFR Y ${ }^{1068}$ phosphorylation.

Figure 5: Known signaling intermediates leading to Akt activation following EGF

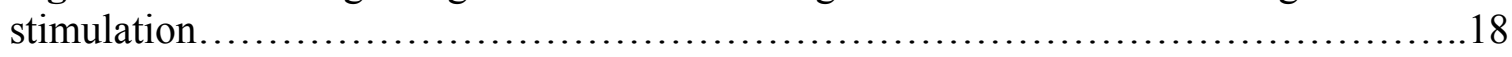

Figure 6: Knocksideways silencing of Clathrin...................................22

Figure 7: EGF-stimulated Akt and Gab1 phosphorylation but not phosphorylation of

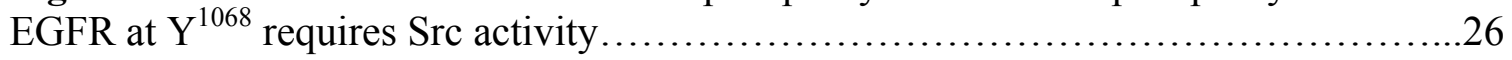

Figure 8: Diagram depicting the domains of TOM1L1_.........................29

Figure 9: Hypothesis-TOM1L1 a possible adaptor regulating early EGFR signaling....31

Figure 10: Expression of pMito-mCherry-FRB and GFP-FKBP-CLCa effectively relocalizes $\mathrm{CHC}$ to mitochondria upon rapamycin addition in RPE cells................43

Figure 11: Knocksideways silencing of clathrin light chain reduces EGF-stimulated Akt phosphorylation in RPE cells....................................................

Figure 12: Expression of pMito-mCherry-FRB and GFP-FKBP-CLCa does not impact EGF-simulated Akt phosphorylation in the absence of rapamycin.......................46

Figure 13: Phosphorylated Gab1 is enriched within plasma membrane clathrin structures

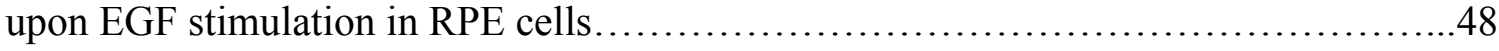

Figure 14: pGab1 is largely restricted to clathrin structures containing EGFR in RPE-wt

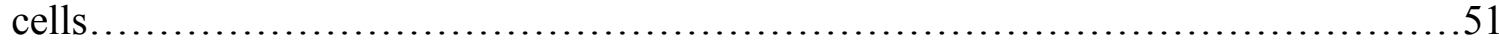

Figure 15: Silencing of TOM1L1 results in reduced Akt phosphorylation...............54

Figure 16: Silencing of TOM1L1 does not affect p42/44 MAPK phosphorylation.......56

Figure 17: Expression of exogenous TOM1L1-GFP and EGFP in RPE cells...........57

Figure 18: Recruitment of TOM1L1-GFP to CCPs................................59 
Figure 19: Clathrin binding mutant of TOM1L1 inhibits gain in EGF stimulated Akt

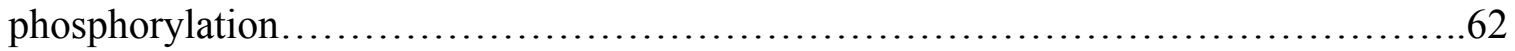




\section{Introduction:}

Overexpression of EGFR is a characteristic of various cancer types, and EGFR drives the growth, proliferation, survival and migration of certain tumors (Salomon et al., 1995). EGFR signaling controls various aspects of cell physiology including cell proliferation, migration, adhesion and apoptosis. Importantly, ligand-bound EGFR activates an intracellular non-receptor serine/threonine kinase, Akt, which controls cell survival and other processes by phosphorylating other effector proteins. Therefore, it is important to understand the regulation of EGFR signaling leading to control of Akt phosphorylation. In this study, I build on previously used experimental approaches to further establish the role of clathrin, in regulating EGFR signaling leading to Akt activation, independent of endocytosis of the receptor. My work further examines the role of a possible regulator of EGFR signaling within CCPs, TOM1L1. Perturbation of clathrin and an adaptor protein TOM1L1 both impair EGF stimulated Akt phosphorylation. Clathrin acts as a scaffold to recruit other adaptor proteins including Gab1. Moreover, the interaction between clathrin and TOM1L1 is crucial to maintain normal Akt activation upon EGF stimulation. Thus, in this introduction, I discuss receptor tyrosine kinases (RTKs), the physiology and signaling of EGFR, control of EGFR signaling and function by clathrin-mediated endocytosis, the novel role of clathrin in the direct regulation of receptor signaling at the plasma membrane, and the TOM family of signaling adaptors. 


\section{Receptor tyrosine kinases (RTKs):}

A major process through which environmental information is transmitted into cells is via activation of protein tyrosine kinases. Several different types of signaling receptors are present at the plasma membrane to transmit extracellular signals, one class of which are receptor tyrosine kinases (RTKs). There are 58 RTKs in humans, with specific ligands to each. RTKs contain extracellular ligand recognition, single membrane spanning, and cytoplasmic protein tyrosine kinase domains. Upon binding of a ligand, most of these receptors dimerize and undergo a series of autophosphorylation events on their cytosolic side and activate various intracellular signaling pathways (Lodish et al., 2000). The ligands for RTKs are soluble or membrane bound peptide/protein hormones including nerve growth factor (NGF), platelet-derived growth factor (PDGF), fibroblast growth factor (FGF), epidermal growth factor (EGF), and insulin (Lodish et al., 2000), each of which bind to specific RTKs. With few exceptions, binding of a ligand to this type of receptor stimulates the receptor's intrinsic proteintyrosine kinase activity, which subsequently stimulates a signal-transduction cascade leading to changes in cellular physiology and/or patterns of gene expression. RTK signaling pathways have a broad range of functions including regulation of cell proliferation and differentiation, promotion of cell survival, and modulation of cellular metabolism (Lodish et al., 200). 


\section{Epidermal growth factor receptor (EGFR):}

Epidermal growth factor receptor (EGFR or ErbB1) belongs to the ErbB family of receptor tyrosine kinases, which along with ErbB2-4 are expressed in cells of different origins, including epithelial, mesenchymal and neuronal cells (Sibilia et al., 1998). Several ligands exist for ErbB receptors; many of these ligands including epidermal growth factor (EGF) are specific to EGFR. EGFR plays a significant role during development. Perinatal lethality is observed in EGFR knockout mice (Sibilia et al., 1998; Threadgill et al., 1995; Sibilia et al., 1995; Miettinen et al., 1995). Multiple defects in organ development during adulthood, including in heart function (Iwamoto et al., 2003) and in mammary development are also characteristics of EGFR knockout in mice generated by using a targeted gene replacement therapy (Stern, 2003). EGF, transforming growth factor (TGF)- $\alpha$ and amphiregulin only bind and activate EGFR and are known as the group I of the EGF ligand family (Wong, 2003).

EGFR controls many aspects of cell physiology, including metabolism, proliferation, survival and adhesion through the activation of intracellular signaling pathways. Rapidly upon binding ligand, EGFR undergoes dimerization and autophosphorylation of it Cterminal tyrosine residues, followed by its internalization from the cell surface. In the active state EGFR forms homodimers (Yarden \& Schlessinger, 1987), it can also form heterodimers with other ErbB family members. Ligand binding stimulates phosphorylation of multiple intracellular tyrosine residues on the C-termini of the EGFR including (including $\mathrm{Y}^{992}, \mathrm{Y}^{1068}, \mathrm{Y}^{1086}$ and $\mathrm{Y}^{1148}$ ) (Downward \& Waterfield, 1984). In addition to the kinase domain of EGFR, c-Src or Src-family kinases (SFK) (Pritchard et 
al., 2008) enhance the extent and repertoire of phospho-tyrosines (pY) on EGFR upon ligand binding. The autophosphorylation initiates a downstream cascade of signaling activation that begins with other proteins containing phospho-tyrosine binding (PTB) and src homology-2 (SH2) domains (or other phospho-tyrosine binding domains) to EGFR (Downward \& Waterfield, 1984). These signaling pathways generate additional secondary signaling intermediates that result in the activation of several different signaling pathways including MAPK, Akt, JAK-STAT and JNK pathways (Oda et al., 2005). The mitogen activated protein kinase (MAPK)/ Extracellular signal-regulated kinase (Erk) pathway controls proliferation, differentiation and survival of many cell types (Orton et al., 2005). Briefly upon activation of EGFR, adaptor protein Grb2 binds to the phosphorylated C-terminal of EGFR and docks a GDP/GTP exchange factor son on sevenless (SOS) (Schulze et al., 2005). SOS then activates a GTPase Ras by displacing a GDP for GTP (Schulze et al., 2005). Upon activation Ras activates a kinase Raf. Raf then activates MAPK/Erk kinase (MEK-1/2) by phosphorylation of two serine residues (Orton et al., 2005). MEK-1/2 then phosphorylates ERK-1/2 at threonine and tyrosine residues (Orton et al., 2005). ERK is a serine/threonine kinase and can regulate gene expression by directly phosphorylating transcription factors such as Myc and Elk (Orton et al., 2005). This leads to the stimulation of cell proliferation, DNA synthesis, cell migration and cell adhesion (Oda et al., 2005) (Figure 1). Another signaling cascade initiated by EGFR is the JAK/STAT pathway, which is also implicated in cell survival responses (Kisseleva et al., 2002; Henson and Gibson 2006). Janus kinase (JAK) phosphorylates Signal Transducer and Activator of Transcription (STAT) proteins localized at the plasma membrane. This leads to the translocation of STAT proteins to the 
nucleus where they activate the transcription of genes associated with cell survival (Kisseleva et al., 2002). While there are numerous signaling proteins activated by EGFR, I will focus on the signalling pathway leading to activation of Akt.

\section{EGFR stimulated activation of Akt:}

Activation of serine/threonine kinase Akt by EGFR regulates cell metabolism, cell survival and proliferation. Thus, Akt serves as an important component of the EGFR signaling pathway. The binding of the adaptor protein Grb2 is facilitated by phosphorylation of Y1068 on EGFR. This allows binding of the adaptor protein Gab1 (Grb2-associated binder) to the Grb2/EGFR complex via its proline-rich domain (PRD) binding to the src homology-3 (SH3) domain of Grb2 (Lock et al., 2000). Gab1 is essential for EGF-stimulated activation of Akt (Mattoon et al., 2004). The recruitment of Gab1 to Grb2/EGFR results in Gab1 phosphorylation on multiple residues including Y446/472/589 (Kiyatkin et al., 2006). Phosphatidylinositol-3-kinase (PI3K), which catalyzes the formation of phosphatidylinositol-(3,4,5)-trisphosphate (PIP3) from phosphatidylinositol-(4,5)-bisphosphate (PIP2), is recruited to the membrane by its $\mathrm{p} 85$ regulatory subunit binding directly to phosphorylated Gab1 on Y446/472/589 (Mattoon et al., 2004; Wong et al., 1996), or indirectly through binding to proteins recruited to phosphorylated Gab1 such as SHP-2 (Knight \& Falke, 2010). The Protein tyrosine phosphatase SHP-2 directly binds phosphorylated Gab1 on Y627/659 (Gu \& Neel, 2003). Therefore, Gab1 is required for EGF-stimulated PI3K activation and Akt phosphorylation, as evinced by the fact that genetic perturbation of Gab1 ablates EGF- 
stimulated Akt phosphorylation (Mattoon et al., 2004). The Gab1- and PI3K-dependent formation of PIP3 at the plasma membrane results in binding and recruitment of the kinases PDK1 and Akt, leading to Akt phosphorylation on T308 and S473 by PDK1 and mTORC2, respectively (Matheny \& Adamo, 2009); both of these phosphorylation events are required for full activation of Akt. Upon activation at the plasma membrane (PM), Akt redistributes to other cellular locales, and controls cellular functions. Activation of Akt leads to the inhibition of inhibitory signaling intermediates such as the TSC $1 / 2$ of the mTORC1 pathway (Burgering, 2003). Akt thus serves to induce a wide variety of transcription factors such as FOXO, GSK3, and S6K, all which are well known activators of cell survival (Burgering, 2003; Cross et al., 1995; Mcmanus et al., 2002).

While the identity of many of the intracellular signaling molecules has been determined, how these secondary signals are coordinated in space and time to achieve control of cell physiology by EGFR remains poorly understood. My research addresses the role of one particular protein, TOM1L1, which together with the protein clathrin, coordinates EGFR signaling in space and time within cells.

The plasma membrane contains many proteins, and these are collectively termed the cell surface proteome. It is a lipid bilayer composed of phospholipids, cholesterol, sphingolipids and various types of proteins. It also acts as a barrier and interface between the cells interior and the extracellular environment by establishing a selective permeability barrier. The plasma membrane consists of integral, peripheral and other associated proteins, and these proteins have diverse functions. Some of these functions 
include receptors for signaling ligands, glycoproteins for identity, transporters, ion channels and adhesion proteins etc. The presence of these proteins on the cell surface also allows for cell-to-cell communications by means of signaling receptors, such as has been discussed for RTKs. Binding of a ligand to a receptor induces a cascade of signaling events within the cell, leading to appropriate response. The cell surface proteome is dynamic, maintained by membrane traffic events that are highly regulated. One of the main mechanisms of regulating this traffic is internalization of cell surface proteins via endocytosis. While there are several known mechanisms of endocytosis (Conner \& Schmid, 2003), the best understood is clathrin-mediated endocytosis (CME), which is the major mechanism of internalization of ligand-bound receptors, including RTKs, from the cell surface (Conner \& Schmid, 2003). As I will describe below, the cellular structures for CME not only control vesicle formation from the cell surface but also form unique microdomains for receptor signaling at the plasma membrane.
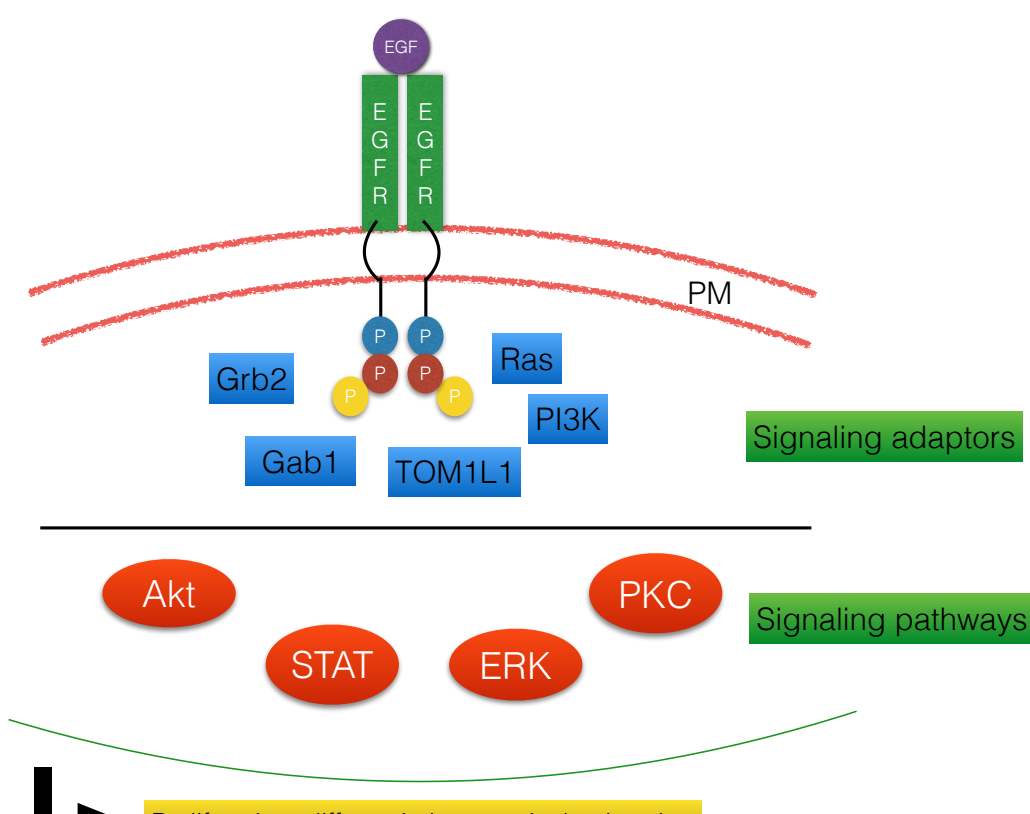

Proliferation, differentiation, survival, migration 
Figure 1: Schematic of EGFR signaling. EGFR controls various aspects of cell physiology by activating different signaling pathways via activation of several adaptor proteins.

\section{Clathrin-mediated endocytosis (CME):}

The main mechanism for the internalization of ligand-bound receptors, transporters and other proteins (collectively called "cargo") from the plasma membrane is clathrin-Mediated endocytosis (CME). Clathrin is a large, $180 \mathrm{kDa}$ protein that is recruited to the plasma membrane; clathrin is required in the formation of vesicles that internalize receptor bound cargo in to the cell during CME. Many molecules such as the Low Density Lipoprotein (LDL) and signaling receptors (EGFR) are internalized via CME (Antonescu et al., 2013). The vesicles generated at the plasma membrane following internalization are either targeted to the lysosomes for degradation or recycled back the to the plasma membrane. By controlling the internalization of membrane proteins from the cell surface, CME acts as a central gatekeeper of the cell surface proteome.

\section{Formation of Clathrin Coated Pits (CCPs):}

CME occurs by the formation of clathrin-coated pits (CCPs), small invaginating regions of the plasma membrane, which eventually lead to formation of an intracellular vesicle for internalization of cargo into the cell. During the formation of CCP, clathrin self assembles to form a lattice and envelops a small region of the plasma membrane (Figure 2A). The CCPs then proceed towards maturation and are then severed from the 
membrane resulting in intracellular vesicles. CCPs are distinctive microdomains, which envelop small regions of the plasma membrane in which specific proteins are concentrated. The assembly, maturation and scission of CCPs followed by the disassembly of clathrin, takes a total of 10-200s (Loerke et al., 2011; Mettlen et al., 2009; Mettlen et al., 2010; Nunez et al., 2011; Jagaman et al., 2008; Loerke et al., 2009; Antonescu et al., 2011; Antonescu et al., 2010). Not all CCPs form intracellular vesicles; Most of the CCPs (50-60\%) are short-lived and abortive, resulting in clathrin disassembly at the plasma membrane (Loerke et al., 2011; Mettlen et al., 2010; Loerke et al., 2009; Antonescu at al., 2010) (Figure 2B).

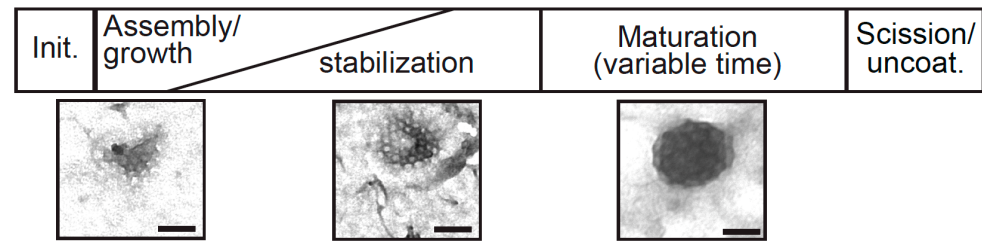

(A) Productive clathrin-coated pits mean lifetimes: $60-80$ s $30-40 \%$ of total clathrin structures

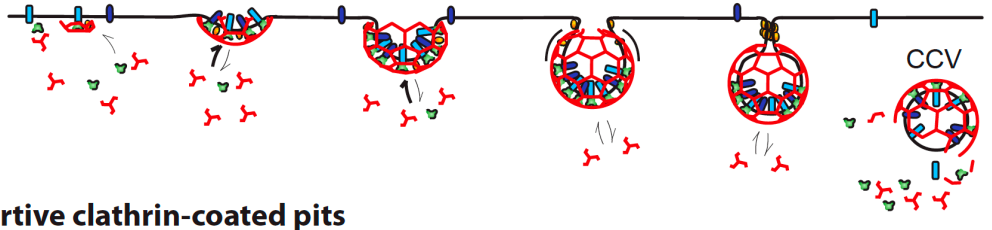

(B) Abortive clathrin-coated pits mean lifetimes: $10-30$ s $50-70 \%$ of total clathrin structures

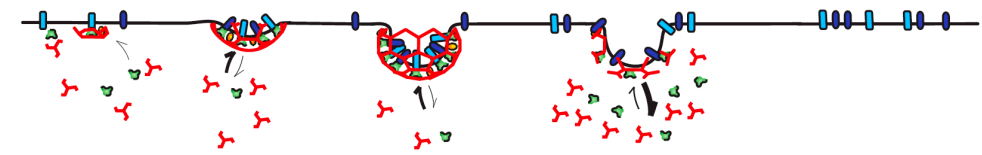

$$
\zeta \text { clathrin } \because \text { AP2 } 00 \text { cargo receptors } 000 \text { dynamin }
$$

Figure 2. Formation of clathrin-coated pits at the plasma membrane. At the plasma membrane, clathrin assembles into either A) Productive clathrin-coated pits (CCPs) that yield intracellular clathrin-coated vesicles (CCVs), B) abortive CCPs that disassemble without internalization (adapted from C.N. Antonescu, unpublished). 
While there are 30-50 proteins (Schmid \& McMahon, 2007) that contribute to CME, there are 4 molecules that represent key interaction "hubs": clathrin, AP-2, dynamin and phosphatidylinositol-(4,5)-bisphosphate (PIP2) (Schmid \& McMahon, 2007), which function to assemble the basic molecular unit of endocytosis at the plasma membrane, the clathrin-coated pit (CCP). CCPs initiate by the action of these four molecules, and are a cargo-containing invaginated region of the plasma membrane of about 50-100 nm.

Clathrin is one of the main proteins involved in CME. Clathrin has a three-legged structure known as the triskelion (Conner \& Schmid, 2003). It consists of three Clathrin heavy chains (CHC), which are associated with three Clathrin light chains (CLC) (Conner \& Schmid, 2003). These chains form a polygonal lattice that aids in the formation of a coated pit on the plasma membrane (Conner \& Schmid, 2003). Clathrin acts like a molecular scaffold to recruit other proteins to the right place so that these can do the "work". CHC consists of an amino-terminal, $\beta$-propeller domain with seven WD40 (beta-transducin) repeats, followed by $42 \alpha$-helical zig-zags of roughly 30 amino acid residues each, a longer $\alpha$-helix, and a 45 -residue carboxy-terminal segment (Kirchhausen et al., 2014). The carboxy-terminal segment contains a short region with an amino acid sequence that is recognized by protein chaperone $\mathrm{Hsc} 70$ and is required for Hsc70-facilitated uncoating. Clathrin light chain (CLC) associates with the heavy-chain, through a long, central $\alpha$-helix (Kirchhausen et al., 2014). The $\mathrm{N}$ terminal domain of CHC interacts with heterotetrameric adaptors including AP-2, via a peptide motif with a 
consensus sequence $\mathrm{L} \Phi \mathrm{X} \Phi \mathrm{D} / \mathrm{E}$, termed as "clathrin box" ( $\Phi$ represents a hydrophobic residue) (Kirchhausen et al., 2014).

Adaptor protein-2 (AP-2) complex is heterotetramer consisting of $\alpha-, \beta 2, \mu 2$ and s-2 adaptin subunits. These subunits assemble into a complex that has ear and core domains (Conner \& Schmid 2003). The core domain is a barrel shaped comprised of the N-termini of two large subunits $\alpha$ - and $\beta 2$ adaptins (Conner $\&$ Schmid 2003). The ear domain is formed by the carboxy- termini of $\alpha$ - and $\beta 2$ adaptins (Conner \& Schmid 2003). The $\alpha$-adaptin targets the AP-2 complex to the plasma membrane via intreraction with phosphatidylinositol $(4,5)$ - biphospahte (PIP2), the $\beta 2$ subunit interacts with clathrin and initiates clathrin assembly (Conner $\&$ Schmid 2003). A medium subunit $\mu 2$ interacts with sorting motifs to concentrate cargo molecules in the pits (Conner \& Schmid 2003). A small subunit sigma-2 structurally stabilizes the core domain (Conner \& Schmid 2003). The ear domain is critical for the recruitment of additional proteins to the membrane and to CCPs. Previous studies have shown that engineering a fusion protein onto the C-terminus of the $\alpha$-adaptin ear domain results in a non-functional protein (Motley et al., 2003), indicating the importance of this domain structurally and functionally to the formation of clathrin lattices, CCPs and to CME.

Phosphatidylinositol (4, 5)- biphospahte (PIP2) is another important molecule that is involved in CME (Padron et al., 2003). It is a phospholipid that binds to and targets the AP-2 complex to the plasma membrane to initiate CME (Padron et al., 2003). PIP2 is thus involved in initiation, stabilization, growth, and maturation of Clathrin Coated Pits (CCPs) (Antonescu et al., 2011). Increased levels of PIP2 might result in formation of CCPs with fewer cargo molecules, thus decreased internalization of the 
cargo (Antonescu et al., 2011). PIP2 expression also regulates the size of the CCP, such that increased PIP2 levels result in CCPs of larger size. Increased PIP2 expression enhances early stages of CCP initiation and growth (Antonescu et al., 2011). PIP2 is also necessary for the assembly and stabilization of CCPs (Antonescu et al., 2011).

Dynamin is GTPase protein required during CME (Conner \& Schmid 2003). It is recruited to the neck of the pinching vesicle, forms a collar around the vesicle and severs the vesicle from the plasma membrane (Conner \& Schmid 2003). Dynamin can selfassemble, and has a GTPase activity that is regulated by self-assembly (Conner \& Schmid 2003). siRNA knockdown of dynamin in cells have resulted in the reduced formation of Clathrin Coated Vesicles (CCVs) and thus reduced internalization of cargo receptors from the cell surface (McFarland et al., 2008) Mutations only in the dynamin gene in Drosophila (Shibire mutation) and Caenorhabditis elegans result in inhibition of internalization of endocytic vesicles into the cell (Conner \& Schmid 2003), indicating that the function of dynamin in the late stages of formation of vesicles at the sites of CCPs is not only essential but is also evolutionarily conserved.

Many early EGFR signals such as those leading to Akt activation exhibit an early and transient activation profile (Kiyatkin et al., 2006). Thus, the time that ligand-bound EGFR occupies CCPs overlaps nearly completely with the activation of early EGFR signals such as those leading to Akt activation.

\section{Regulation of EGFR by Clathrin:}


Rapidly upon binding ligand, EGFR undergoes recruitment into CCPs, and this eventually leads to receptor internalization from the cell surface via Clathrin Mediated Endocytosis (CME). EGFR signaling can be differentiated in timing and hierarchy. The regulation of EGFR signaling can be distinguished in three different phases: 1) Early, 2) Intermediate and 3) Late phase of EGFR signaling. Early EGFR signaling occurs within the first few minutes of EGFR activation where the receptor is largely at the PM. Intermediate signaling can be characterized as minutes to hours after EGFR activation, during which time the receptor is largely in endosomes and en route to degradation/recycling. Finally, the late phase of EGFR signaling, which occurs hours to days after receptor activation and affects include gene expression, differentiation, migration, etc. Hence, the initial phases of (within 10 min of ligand addition) EGFR signaling occurs while the receptor is localized at the plasma membrane within clathrincoated pits, and is required for normal engagement of intermediate and late stages of EGFR signaling. That early EGFR is functionally required for all subsequent aspects of EGFR signaling, Antonescu lab was thus motivated to ask whether clathrin was required for early EGFR signaling.

Indeed, siRNA gene silencing of clathrin heavy chain (CHC) alters EGFR intracellular signaling, suggesting that CME controls EGFR function (Figure 3). While cells treated with control siRNA exhibited robust phosphorylation of Gab1 and Akt upon EGF stimulation, cells treated with CHC siRNA had a markedly blunted response (Figure 3). Moreover, other studies analyzed the effect of defective endocytosis on the EGFR signaling: cell proliferation dependent on EGF was enhanced in the cells with defective endocytosis mechanism (Kiyatkin et al., 2006), suggesting that CME is important in 
attenuating EGFR signaling (Amandio et al., 1996). Another study indicated that EGFR internalized via $\mathrm{CME}$ are recycled back to the plasma membrane from the endosome, whereas internalization of EGFR via Clathrin-independent endocytosis (CIE) targets it for degradation to the lysosomes (Sigismund et al., 2008). CME sustains EGFR signaling and determines the duration of some EGFR activated pathways (Sigismund et al., 2008). CME not only mediates attenuation of EGFR signaling but also plays a critical role in controlling specific signaling pathways.

While these previous studies have examined the role of EGFR internalization to endosomes in the regulation of EGFR signaling, recent unpublished observations from the Antonescu laboratory has shown that EGFR recruitment to CCPs, but NOT receptor internalization to endosomes, controls the activation of specific signaling pathways following EGF stimulation. These experiments relied on comparing the effects of siRNA gene silencing of clathrin heavy chain (CHC) to that of dynamin (Dyn). Silencing of CHC prevents formation of CCPs at the cell surface, while perturbation of Dyn allows CCPs to form and recruit cargo (such as EGFR), but prevents their internalization from the cell surface. Hence, while both dyn2 and CHC siRNA treated cells force EGFR to signal from the plasma membrane, dyn2 siRNA results in EGFR signaling from within CCPs while CHC siRNA from other microdomains of the plasma membrane. siRNA gene silencing of CHC prevented EGF-stimulated Gab1 and Akt phosphorylation. Importantly, silencing of Dyn did not prevent Akt activation (Figure 3). The activation of Erk phosphorylation of EGF stimulation was unaffected by either $\mathrm{CHC}$ or dyn 2 silencing (Figure 3, lower green panel). These results suggest that incorporation of EGFR within 
clathrin-coated pits at the cell surface but not the internalization of the receptor, are required for activation of Akt.

These observations led to the working hypothesis of the Antonescu lab that CCPs are clathrin-coated microdomains, enriched in specific factors (proteins, lipids), that are required for activation of specific signaling pathways by receptor tyrosine kinases such as EGFR.

The molecular mechanism by which clathrin regulates EGFR signalling at the plasma membrane is not known. Examining this function of clathrin is the aim of my research. How might clathrin function in EGFR signalling?

Pitstop2 is a cell-permeable small molecule that competitively inhibits protein binding to $\mathrm{CHC}$ at its N-terminus (von Kleist et al., 2011). Treatment of cells with pitstop2 allows formation of CCPs, yet prevents the recruitment of specific proteins to CCPs (von Kleist et al., 2011). Consistent with the siRNA results, RPE cells treated with pitstop2 exhibited a selective reduction in EGF-stimulated Gab1 and Akt phosphorylation, but not in that of EGFR (Figure 4). Hence, this identifies CCPs as critical components of EGF-stimulated Akt activation. Moreover, since pitstop2 inhibits normal clathrin function by competitive binding to the $\mathrm{N}$-terminal protein interaction hub of clathrin, this also suggests that binding of one or more proteins directly to $\mathrm{CHC}$ may be required to provide CCPs with the capacity to facilitate EGF-stimulated Akt phosphorylation, although the impact of pitstop treatment on CCPs is likely to have broader consequence on clathrin scaffold assembly as well. This demonstrates that EGFR localization within functional CCPs, but not receptor internalization is required for 
activation of early EGFR signaling events. Since pitstop2 blocks binding of cytosolic effectors to clathrin I hypothesize that the role of clathrin in regulating EGFR signaling is to recruit a cytosolic effector.

This hypothesis is supported by the findings of two previous studies, which uncovered that an EGFR harboring 24-point mutations that prevented receptor recruited to CCPs upon binding ligand resulted in reduced EGF-stimulated Akt activation (Goh et al., 2010). However, the reduction in Akt activation might also be attributed to the large number of mutations introduced, rather than failure to be recruited to the CCPs. As reported by Di Fiore \& col. EGF-stimulated Akt phosphorylation was reduced upon silencing of clathrin heavy chain (Sigismund et al., 2008). However, this study examined mostly long term signaling regulation ( $>10$ mins of ligand stimulation) and did not compare the effects of clathrin silencing to those of dyn2 silencing; hence, it could not resolve a requirement for EGFR localization to CCPs from EGFR localization to endosomes.

Collectively, these previous experiments have revealed that early EGFR signaling requires CCPs but not receptor internalization. This indicates that recruitment of the receptor to clathrin-coated membrane microdomains is required to locally activate certain early EGFR signal intermediates leading to Akt activation, which include Grb2, Shc, Gab1, SFKs, PI3K, PIP3, and PDK1 (Figure 5). Significant gaps still remain in our understanding of early EGFR signaling leading to Akt activation.

What is the spatial, temporal, and functional hierarchy of these signals and how do clathrin-coated membrane microdomains function in the EGF-stimulated 
activation of Akt?

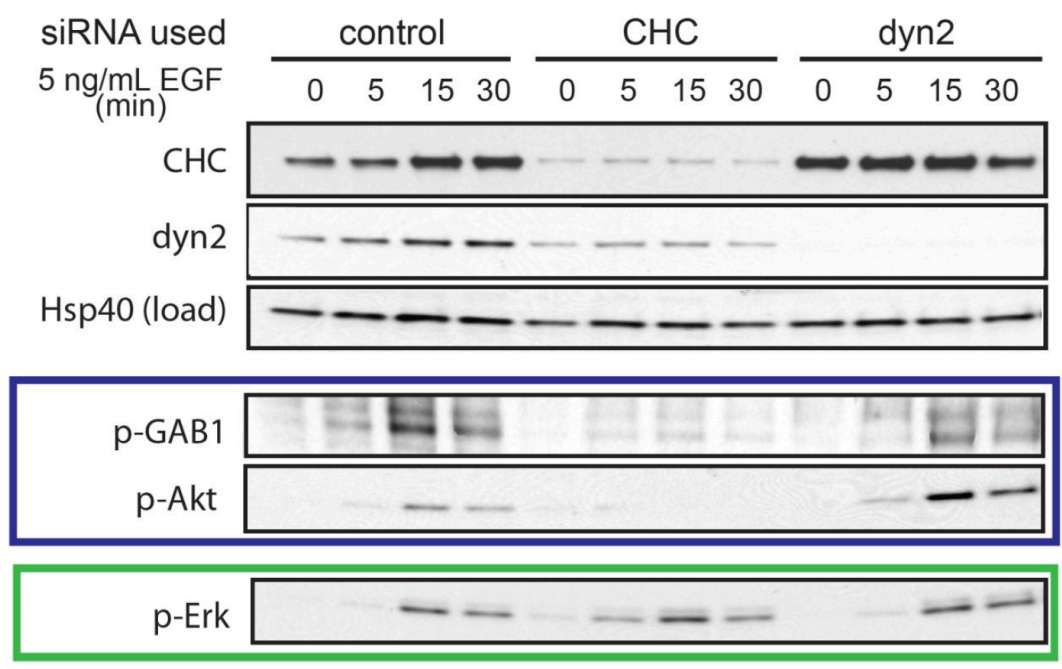

Figure 3. Silencing of CHC or Dyn2 results in similar inhibition of EGFR endocytosis yet distinct effects on EGF-stimulated Akt activation. RPE cells were treated with siRNA targeting CHC, dyn2 or unrelated (control) siRNA. Cells were then stimulated with $5 \mathrm{ng} / \mathrm{mL}$ EGF for the indicated times. Shown are immunoblots of whole-cell lysates using anti-CHC, anti-dyn2, or anti-Hsp40 (loading control), or antibodies recognizing the phosphorylated (activated) forms of Gab1, Akt or Erk. Shown in the blue box are signals (phospho-Gab1, phospho-Akt) that are reduced upon silencing of CHC but not dyn2. Shown in the green box are signals (phospho-Erk) that are unaffected by silencing of either $\mathrm{CHC}$ or dyn2. (Camilo Garay and C. N. Antonescu unpublished data).

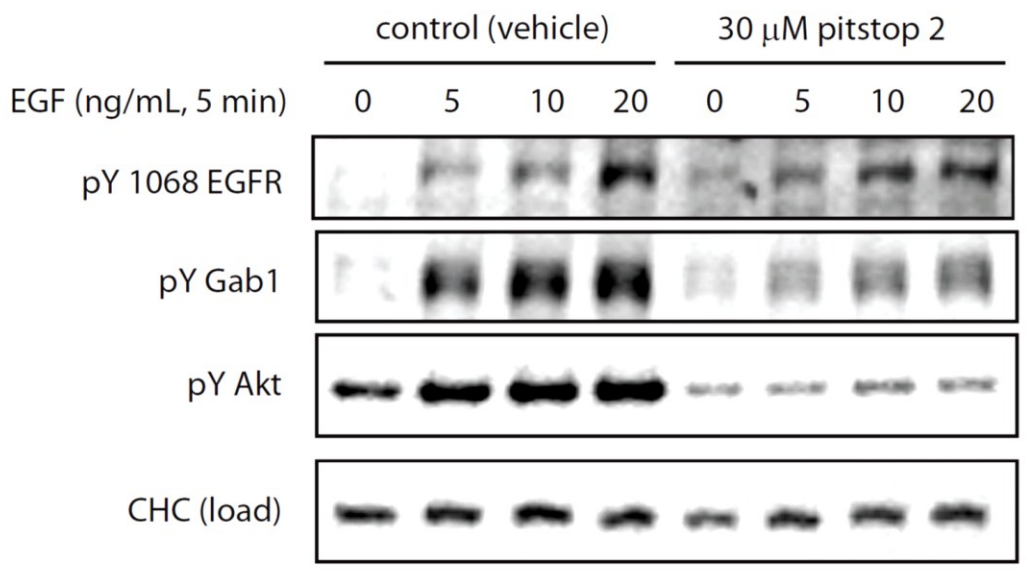

Figure 4. Treatment of RPE cells with the CHC-inhibitor Pitstop2 prevents EGF-stimulated Gab1 and Akt phosphorylation but not EGFR Y ${ }^{1068}$ phosphorylation. RPE cells were treated with $30 \mathrm{uM}$ pitstop2 (Abcam, Cambridge, MA, USA) or with $0.1 \%$ DMSO vehicle control for $30 \mathrm{~min}$. Cells were then stimulated with EGF for $5 \mathrm{~min}$ at the concentration indicated $(\mathrm{ng} / \mathrm{mL})$. Shown are immunoblots of wholecell lysates, probed with anti-phospho-EGFR, anti-phospho-Gab1, anti-phospho-Akt or anti-CHC (loading control). (Camilo Garay and C. N. Antonescu unpublished data). 


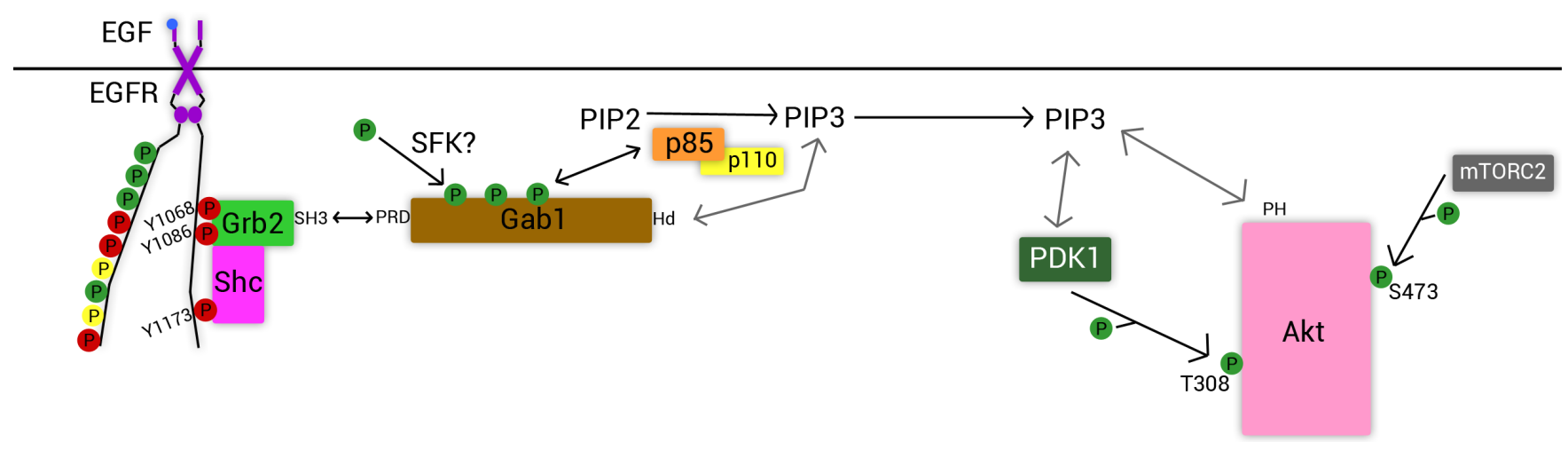

Figure 5: Known signaling intermediates leading to Akt activation following EGF stimulation.

Before examining the molecular mechanism by which clathrin may control receptor signaling, I sought to first obtain further evidence of the role of clathrin as a regulator of EGFR signaling at the plasma membrane. This is due to the fact that the two experimental strategies to perturb clathrin, siRNA gene silencing and pharmacological inhibition (pitstop) each had some limitiations. Perturbation of CHC and Dyn using siRNA gene silencing over several days, which could result in cellular perturbations in addition to those affecting formation of CCPs and their scission from the PM. Pitstop2, while having the advantage of being acute, suffers from many of the same concerns of the use of drugs, specifically, that of possible off-target effects. Hence, I sought to complement these two strategies with a novel method to inhibit CME that was both specific and acute. 


\section{Inhibition of Clathrin function:}

A summary of the previously used methods to inhibit endocytosis can be found in table 1 , as well as their limitations as these relate to the study of the function(s) of clathrin in cells.

Table 1: A summary of the previously used methods to inhibit endocytosis.

\section{Methods To Inhibit Clathrin Mediated Endocytosis (CME)}

Genetic or molecular biology:

Expression of cDNA constructs with mutations in Clathrin, dynamin-2, AP180 protein (Dutta et al., 2012). siRNA silencing of CHC, AP-2 complex, dynamin (Dutta et al., 2012).

\section{Limitations:}

Take several hours $>\sim 72$, cannot assay the phenotype (Dutta et al., 2012).

Cell may utilize compensatory mechanisms such as Caveolin-mediated endocytosis or clathrin independent endocytosis
Acute, chemical:

Cytosolic Acidification (Dutta et al., 2012), (targets vesicle budding)

Hypotonic Treatment (Dutta et al., 2012), (Clathrin dissociation)

\section{Drugs:}

Pitstop (block ligand access to the clathrin terminal domain) (Dutta et al., 2012).

Chlorpromazine (induces clathrin misassembly) (von Kleist \& Haucke, 2011)
Limitations:

Non-specific to CME (Dutta et al., 2012).

\section{Limitations:}

Non-specific to CME (Dutta et al., 2012).

\section{Previous approaches of CME inhibition:}

Genetic and molecular methods to perturb CME: Many approaches and methods have been developed and directed towards the inhibition of CME. Expressing the mutant forms of the proteins involved in CME, such as dynamin-2, AP180 protein and the 
clathrin chain are effective to a great extent at inhibiting CME, as these can act in a dominant-interfering manner (Dutta et al., 2012). In recent years, siRNA silencing of the CHC, AP-2 complexes and dynamin have proved to inhibit CME (Dutta et al., 2012). Despite the effectiveness of these genetic and molecular techniques, they have limitations as it might take several hours or days to come into effect (Dutta et al., 2012). This might lead to the stimulation of other compensatory mechanisms or cellular responses since clathrin is also involved in budding from the golgi, lysosomes, endosomes and cell division, thus complicating the interpretation of results (Dutta et al., 2012). Altered CHC function might impact RTK signaling as it will impact the cell surface receptor levels.

Acute treatments leading to inhibition of CME: More acute methods including cytosolic acidification and hypotonic treatment have been shown to block CME, although these methods are acute, they may be quite non-specific to CME, as they also inhibit Clathrin Independent Endocytosis (CIE) (Dutta et al., 2012). Most recently a drug called Pitstop-2 was designed to inhibit clathrin terminal domain (CTD) in order to study its role in formation of CCPs dynamics (von Kleist et al., 2011). Pitstop-2 was believed to be a specific inhibitor of CME (von Kleist et al., 2011). As it turns out, another study showed that pitstop-2 when used at 30 um is a potent inhibitor of CIE (Dutta et al., 2012). CD59, a GPI-anchored protein, normally internalized by CIE, exhibits reduced internalization in the presence of pitstop-2. Internalization of other cargo proteins (CD44, CD98, CD147), normally endocytosed by CIE, was blocked by pitstop-2 treatment as well(Dutta et al., 2012). Despite the fact that we examined the function of clathrin as a regulator of EGFR signaling at lower $(5 \mathrm{uM})$ concentrations of pitstop2 where off-target effects are likely to 
be less pronounced, to further understand $\mathrm{CME}$ and its role in signal transduction, an acute yet specific method was required to inhibit CME.

\section{Rapamycin induced heterodimerization (aka Knocksideways silencing) as a tool to achieve inhibition of specific proteins:}

Rapamycin was first discovered as a drug that binds to and inhibits mTOR (mechanistic Target of Rapamycin). This inhibition of mTOR results from heterodimerization of mTOR with a prolyl isomerase FKBP12 upon addition of rapamycin. This drug-induced interaction between domains from each of these proteins has been used in several studies to determine the function of specific proteins. Briefly, knocksideways silencing exploits the rapamycin-inducible heterodimerization of the FKBPdomain of FKBP12 with the rapamycin binding (FRB) domain of mTOR. The protein to be inhibited is modified by its fusion to one of the rapamycin binding domains (FKBP), while the other rapamycin binding domain (FRB) is fused to a mitochondrial targeting sequences (from the mitochondrial outer membrane protein TOM70p) (Robinson et al., 2010). Upon addition of rapamycin, there is a redistribution of the protein fused to FKBP to the mitochondria, resulting in the sequestration of that protein away from other cellular locales, thus effectively inhibiting its function (Figure 6). This strategy was used to effectively acutely silence AP-2 function by tagging the FKBP domain to AP-2 (Robinson et al, 2010), thus perturbing assembly of CCPs.

Subsequent work has used a similar strategy to fuse clathrin light chain (CLC) to GFP and the FKBP heterodimerization domain (GFP-FKBP-CLCa); this construct can be 
readily expressed in cells along with the mitochondria-targeted FRB heterodimerization domain (pMito-mCherry-FRB, henceforth MitoTrap) (Cheeseman et al. 2013). This approach will be used to validate the results of the siRNA and pharmacological approaches on clathrin inhibition.
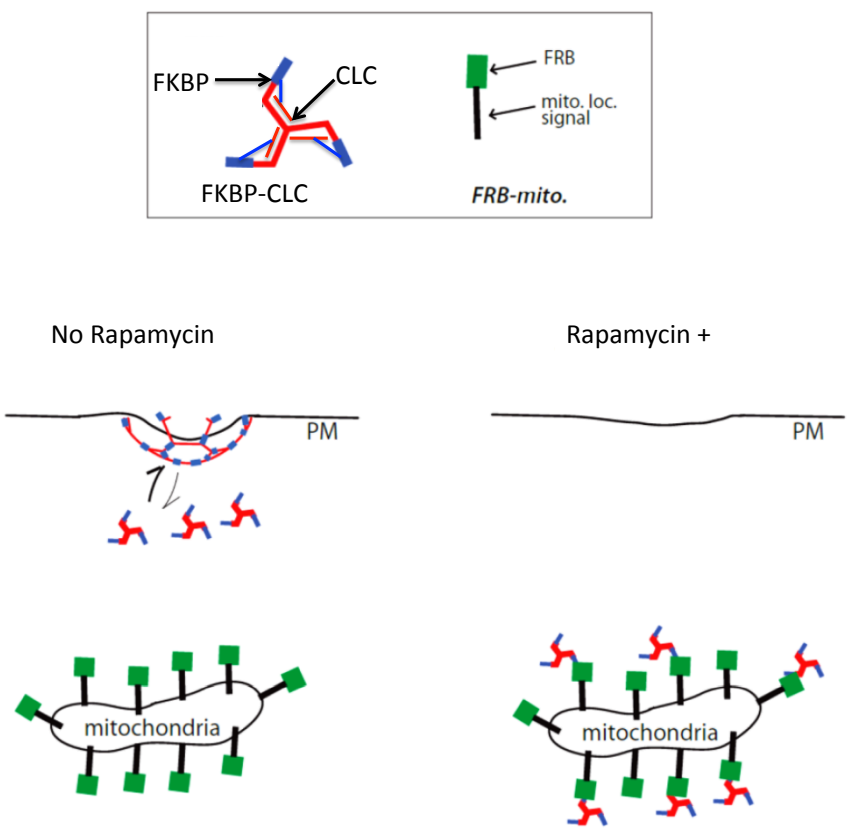

Figure 6: Knocksideways silencing of Clathrin. In the absence of rapamycin clathrin is normally localized across the cell. Upon addition of rapamycin clathrin is sequestered to mitochondria by heterobimerization of the FKBP and FRB domains.

I hypothesize that knocksideways silencing of clathrin will similarly impair EGFstimulated Akt phosphorylation, as was observed for perturbation of clathrin by siRNA gene silencing and pharmacological inhibition. This leads to the question of the mechanism by which clathrin may control EGFR signaling leading to Akt phosphorylation. Since clathrin is not a kinase itself, and yet EGFR signaling leading to Akt phosphorylation requires the action of tyrosine kinases (to phosphorylate EGFR and Gab1), it is possible that clathrin functions by scaffolding a non-receptor tyrosine kinase to facilitate EGFR signaling. 
As described earlier, some Src family kinases (SFKs) are activated in some conditions and cells upon EGFR ligand binding. Some evidence suggests that Src regulates the phosphorylation of signaling intermediate Gabl eventually leading to Akt activation, as I describe below.

\section{Src Family Kinases (SFKs) are required for EGF-stimulated Gab1 and Akt phosphorylation:}

Src family kinases (SFK) belong to the subfamily of cytoplasmic tyrosine kinases and are comprised of eight members, three of which (Src, Fyn, and Yes) are widely expressed (Franco et al., 2006). SFK share a common structure, including a myristoylation site at the $\mathrm{N}$ terminus for membrane targeting, a unique sequence followed by an SH3, an SH2 and a kinase domain (Franco et al., 2006). In addition, they all contain an autophosphorylation site (Tyr416) in the activation loop of the catalytic domain for kinase activation and a Tyr residue in the short C terminus (Tyr527) that, when phosphorylated by Csk, inhibits enzymatic activity (Franco et al., 2006). The cytoplasmic tyrosine kinases of the Src family (SFK) play important roles in signal transduction induced by growth factors leading to DNA synthesis, cytoskeletal rearrangement, and receptor endocytosis (Bromann et al., 2004).

Since EGF-stimulated Akt phosphorylation is reduced by inhibition of SFKs (Kassenbrock et al., 2002), preliminary experiments examined whether SFK inhibition also leads to perturbation of upstream signals. Previous work in the Antonescu lab showed that both the SFK inhibitor PP2 and the EGFR kinase inhibitor AG1478 ablated 
the EGF-stimulated phosphorylation of Gabl and Akt, whereas that of EGFR $\mathrm{Y}^{1068}$ was insensitive to PP2 (Figure 7). Therefore, the requirement for SFKs in EGF-stimulated Akt activation occurs in signals downstream of Grb2 binding to the $\mathrm{pY} \mathrm{Y}^{1068}$ on EGFR, and prior to Gab1 phosphorylation (Figure 5).

The requirement for SFKs in EGF-stimulated Akt activation may reflect the need for a direct phosphorylation of Gab1 by SFKs, as shown for PDGF-stimulated Gab1 phosphorylation(Chan et al., 2003). The requirement for EGFR localization to CCPs for early receptor signaling leading to Akt activation indicates that CCPs are critically enriched in signaling intermediates.

\section{Clathrin: A protein scaffold:}

Several proteins are recruited to the CCPs during their formation. CME is a dynamic process and requires several proteins and binding factors; however, in the absence of functional clathrin heavy chain and AP-2, clathrin-coated pits are virtually absent and clathrin-mediated endocytosis is ablated (Motley et al., 2006; Antonescu et al., 2008). As a result of this, several previous studies have employed siRNA gene silencing of either clathrin-heavy chain or AP-2 subunits in order to perturb CME. 
Table 1: Adaptor proteins with direct interaction with clathrin assembly and their role in signaling regulation

\begin{tabular}{|c|c|c|}
\hline Protein & $\begin{array}{l}\text { Direct Binding to Clathrin } \\
\text { components }\end{array}$ & Regulation of signaling \\
\hline TOM1L1 & $\mathrm{CHC}$ & $\begin{array}{l}\text { Binds to ubiquitinylated } \\
\text { proteins via GAT domain } \\
\text { (Wang et al., 2010) } \\
\text { Binds and activates Src (Liu } \\
\text { et al., 2009; Collin et al., } \\
\text { 2007) } \\
\text { Binds to Grb2 (Liu et al., } \\
2009 \text { ) }\end{array}$ \\
\hline Grb2 & SH3-PRD interactions & $\begin{array}{l}\text { SH3 domains bind EAPs, } \\
\text { SH2 domain binds pY on } \\
\text { EGFR (Holgado-Madruga } \\
\text { et al., 1996), required for } \\
\text { EGFR localization to CCPs } \\
\text { (Jiang et al., 2003; } \\
\text { Johannessen et al., 2006) } \\
\text { Grb2 facilitates EGF- } \\
\text { stimulated activation of } \\
\text { Ras-MAPK (Holgado- } \\
\text { Madruga et al., 1996; Jiang } \\
\text { et al., 2003) } \\
\text { Grb2 interacts with Shc, } \\
\text { Gab1 (Sarmay et al., 2006) }\end{array}$ \\
\hline Disabled-2 (Dab2) & $\alpha$-ear of AP2 & $\begin{array}{l}\text { Binding of dab2 to Grb2 } \\
\text { disrupts interaction between } \\
\text { Sos and Grb2, negatively } \\
\text { regulates MAPK pathway } \\
\text { (Zhou \& Hsieh, 2001) }\end{array}$ \\
\hline CIN85 & $\alpha$-ear of AP2 & $\begin{array}{l}\text { Binds } \mathrm{p} 85 \text { subunit and } \\
\text { negatively regulates PI3K } \\
\text { activity } 100\end{array}$ \\
\hline Intersectin & AP-2 & $\begin{array}{l}\text { Harbours SH3 domains } \\
\text { which bind Sos and } \\
\text { stimulate Ras } \\
\text { activation (Tong et al., } \\
\text { 2000) } \\
\text { Harbours SH3 domains } \\
\text { which Cbl, enhances Cbl- } \\
\text { mediated } \\
\text { ubiquitinylation } \\
\text { (Nikolaienko et al., 2009) }\end{array}$ \\
\hline
\end{tabular}




\begin{tabular}{|l|l|}
\hline & $\begin{array}{l}\text { Binds and activates PI3K- } \\
\text { C2-betaleading to Akt } \\
\text { activation (Das et al., 2007) } \\
\text { Harbours Cdc42 GEF } \\
\text { activity and regulates actin } \\
\text { cytoskeleton }\end{array}$ \\
\hline
\end{tabular}

Clathrin and AP-2 are recruitment hubs for many other proteins identified by systematic protein interaction studies (Schmid \& McMahon, 2007), several of which have properties of signaling regulators. The protein that critically bridges EGFR activation and Gab1 phosphorylation should exhibit the following: 1) recruitment to CCPs dependent on interaction with clathrin, 2) possible interaction with EGFR to facilitate the process, and 3) interaction with Src or SFKs. Based on the requirements, there is a candidate protein that satisfies the interactions mentioned above. Target of Myb-1 like protein 1 (Tom1L1).

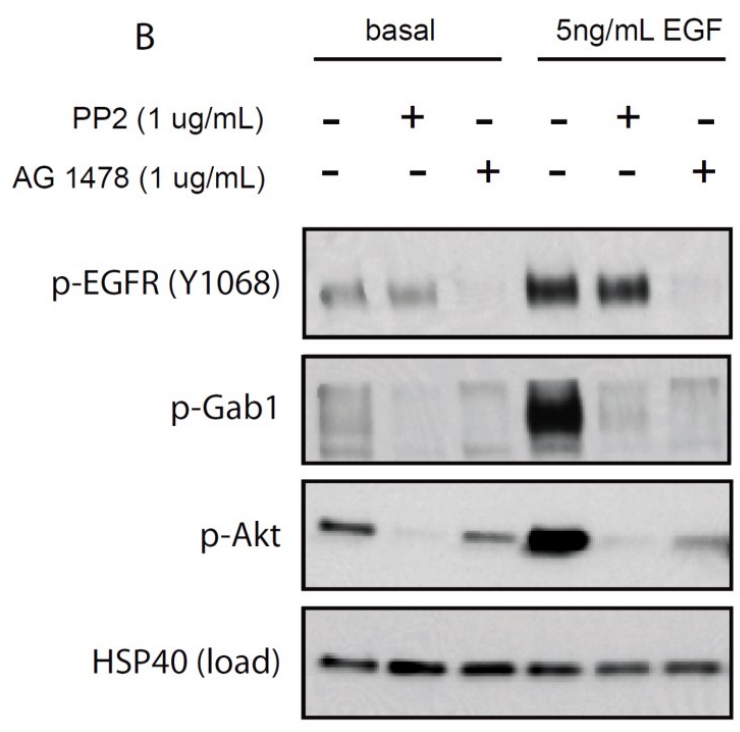

Figure 7: EGF-stimulated Akt and Gab1 phosphorylation but not phosphorylation of EGFR at $\mathrm{Y}^{1068}$ requires Src activity. RPE cells were treated with $1 \mathrm{ug} / \mathrm{mL}$ PP2, $1 \mathrm{ug} / \mathrm{mL}$ AG1478 or with $0.1 \%$ DMSO vehicle control for $30 \mathrm{~min}$. Cells were then stimulated with $5 \mathrm{ng} / \mathrm{mL}$ EGF for $5 \mathrm{~min}$. Shown are immunoblots of whole-cell lysates, probed with anti-phospho-EGFR, anti-phospho-Gab1, anti-phosphoAkt or anti-Hsp40 (loading control). (Camilo Garay and C. N. Antonescu unpublished data). 


\section{TOM1L1:}

Target of Myb-1 like protein 1(Tom1L1) also known as Src activating and signaling molecule (SRCASM) in murine system, is part of the Vps27/Hrs/Stam (VHS) domain-containing family of proteins, which also include Hrs, Stam1, Stam2, GGA1, GGA2, GGA3, Tom1 and Tom1L2 (Bonifacino, 2004; Lohi et al., 2002). Characterized by an N-terminal VHS domain followed by a central GGA and Tom (GAT) domain and a C-terminal region, Tom1, Tom1L1 and Tom1L2 together form a subfamily (Tom) of VHS domain proteins. Amongst these three proteins, Tom1L1 is the most divergent. Tom 1 and Tom1L2 are about $59 \%$ identical to each other, while Tom $1 \mathrm{~L} 1$ shares only 30 and $31 \%$ amino acid sequence identity with Tom1 and Tom1L2, respectively (Wang et al., 2010). The homology of Tom1L1 with Tom1 and Tom1L2 is confined to the N-terminal VHS and the central GAT domains, while its C-terminal region is relatively distinct from the homologous Tom1 and Tom1L2 C-terminal region (Wang et al., 2010). In addition to TOM1L1 both TOM1 and TOM1L2 are known to have interactions with clathrin and endosomal proteins (Wang et al., 2010). However, TOM1L1 is the only one that is implicated in signalling as it binds SFKs. Since TOM1L1 is involved in both protein trafficking and signalling, it makes TOM1L1 an excellent candidate to study early EGFR signalling. TOM1L1 is a clathrin N-terminal domain binding protein, which satisfies the first requirement of a clathrin-effector protein. Tom1L1 is Tyr-phosphorylated by the Src family kinases and may have a negative function in Src kinase signaling (Seykora et al., 
2002; Franco et al., 2006). Furthermore, Tom1L1 can interact with Hrs and TSG101/VPS23, which are important for sorting of endocytosed EGFR into multivesicular bodies (MVBs) for delivery to the lysosome for degradation (Puertollano et al., 2005). The adaptor protein TOM1L1 may participate in the internalization of EGFR via CME upon EGF stimulation, and is subsequently distributed to the endosomes (Liu et al., 2009). Interaction of TOM1L1 with CHC N-Terminus has been reported to regulate the membrane partitioning and localization of the tyrosine kinase Src. Tom1L1$\mathrm{CHC}$ complex is able to reduce the incorporation of Src kinases into the caveolae, thereby preventing its association with the PDGF receptor that is required for the induction of mitogenesis (Franco et al., 2006; Collin et al., 2007). The Tom1L1-CHC complex is also able to reduce the level of oncogenic Src in cholesterol-enriched microdomains, thus implying a novel mechanism involved in negative regulation of mitogenic and transforming signals by modulating SFK partitioning at the plasma membrane (Franco et al., 2006; Collin et al., 2007).

TOM1L1 1) binds CHC directly via $\mathrm{F}^{449}$ DPL motif, 2) binds Grb2 via a $\mathrm{Y}^{392}$ DNF motif, forming an EGFR-TOM1L1 complex (Liu et al., 2009; Wang et al., 2010; Collin et al., 2007), and 3) binds and activates SFKs via $\mathrm{P}^{421} \mathrm{PL}, \mathrm{Y}^{457} / \mathrm{Y}^{460}$ EEID motifs (Collin et al., 2007; Li et al., 2005) (Figure 8). EGF stimulation redistributes TOM1L1 from the cytosol to the PM and subsequently to endosomes (Liu et al., 2009). Overexpressed TOM1L1 co-localizes with clathrin in punctate structures throughout the cell (Collin et al., 2007) and enhances SFK and MAPK activation in the absence of EGF (Collin et al., 2007). However, whether TOM1L1 is present within CCPs at the PM or 
within endosomes (given that it interacts with the endosomal adaptors Hrs) is not known, given the lack of suitable resolution of clathrin structures in previous studies.

In addition to reports of TOM111 functioning to negatively regulate the activity of some SFKs under some circumstances, TOM1L1 also regulates sustained signaling upon prolonged stimulation with EGF (Wang et al., 2010). TOM1L1 binds to intracellular clathrin adaptor Hrs, and overexpression of an Src-binding mutant of TOM1L1 delays EGF-stimulated EGFR degradation. TOM1L1 overexpression reduces MAPK activation following 30 min of EGF stimulation(Collin et al., 2007), when EGFR is found in intracellular membranes (Sorkin and Goh, 2009). Hence, TOM1L1 has the capability to interact with clathrin, but whether it interacts with clathrin within CCPs at the PM in order to be localized to these structures is not known. Moreover, whether TOM1L1 has a functional role in clathrin-dependent EGFR signaling leading to Akt phosphorylation is not known.

From this work emerge two questions:

Is TOM1L1 required for EGF-stimulated Akt phosphorylation, and does an interaction between TOM1L1 and clathrin underlie the role of TOM1L1 in regulating

\section{EGFR signaling?}

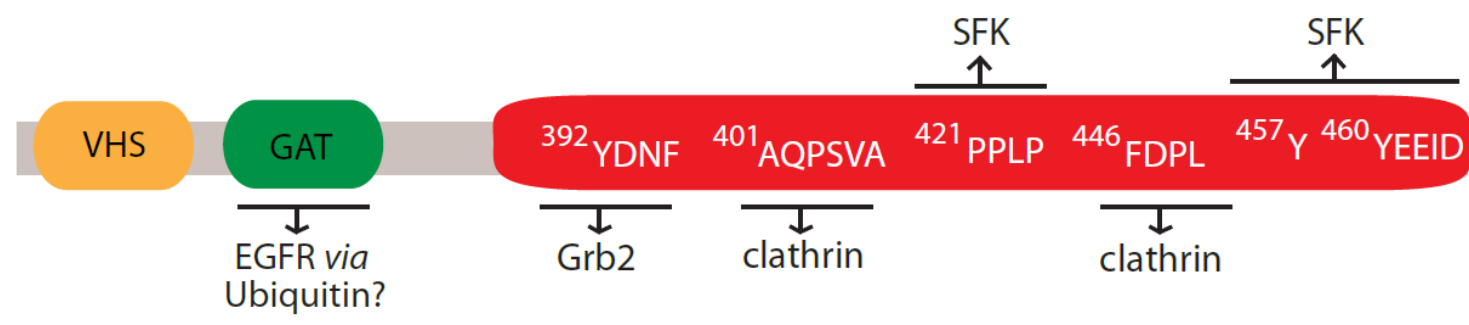

Figure 8: Diagram depicting the domains of TOM1L1 (VHS, GAT, C-terminal domains) and known interactions of TOM1L1 motifs with Grb2, EGFR, CHC and c-Src (adapted from C.N. Antonescu, 
unpublished).

Within the context of the known function and properties of EGFR signaling and regulation thereof by clathrin, emerges the overall rationale and hypothesis for my thesis, as follows:

\section{Overall Rationale:}

Receptor-proximal, early EGFR signaling occurs while the receptor is largely inside clathrin-coated pits at the plasma membrane. Previous work in the lab showed using siRNA gene silencing and pharmacological inhibitors that clathrin is required for EGFR signaling. However, given the limitations of the methods used to perturb clathrin, an additional method is required to conclusively demonstrate that clathrin is required for EGFR signaling. More importantly, the molecular mechanism by which clathrin controls EGFR signaling is not known.

\section{General Hypothesis:}

I hypothesize that requirement for clathrin but not receptor endocytosis in EGFR signaling is due to the ability of clathrin to bind and recruit TOM1L1, which functions to activate SFKs and to localize these kinases in close proximity to other EGFR bound signaling adaptors, thus facilitating EGFR signaling leading to Akt phosphorylation. 


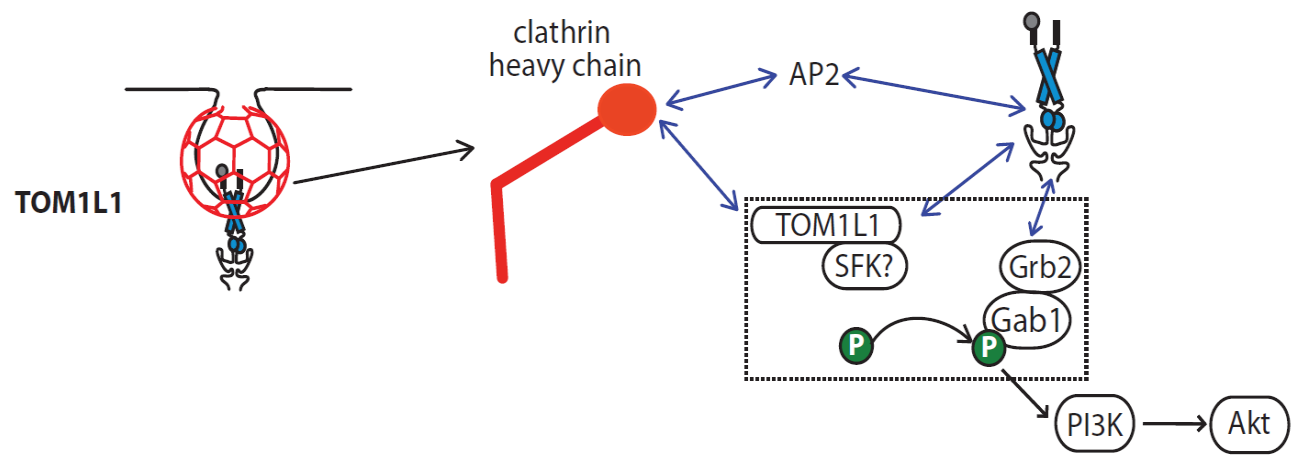

Figure 9: Hypothesis-TOM1L1 a possible adaptor regulating early EGFR signaling. TOM1L1 might be the probable adaptor protein resulting in complete activation of Gab1 via SFKs upon EGF stimulation, thereby leading to Akt phosphorylation (adapted from C.N. Antonescu, unpublished). 


\section{Materials and methods:}

\section{Antibodies and probes:}

Anti phospho-EGFR (pEGFR Y1068), anti phospho-MAPK42/44 and anti-pan-Akt were form were from Cell Signaling Technologies (Danvers, MA); anti-phospho-Akt (pS473) antibodies and Alexa-555 conjugated EGF (A555-EGF) were from Life Technologies (Carlsbad, CA). Anti-actin and anti-clathrin heavy chain (X-22) were from Santa Cruz Biotechnologies (Santa Cruz, CA); anti phospho-Gab1 (pY627) was from Applied Biological Materials, (Richmond, BC). Fluorophore conjugated or horseradishperoxidase secondary antibodies were obtained from Jackson Immunoresearch (West Grove, PA). DMSO and EGF (5ng/ml) for stimulation experiments, unless otherwise noted) were purchased from Life Technologies, Inc. (Carlsbad, Ca).

\section{Cell lines and Cell culture:}

Wild-type human retinal pigment epithelial cells (RPE-wt), and RPE cells expressing clathrin light chain-GFP (RPE-GFP), and clathrin light chain-RFP (RPE-RFP) were cultured in Dulbeccos's modified Eagle's medium (DMEM/F12) (with glucose and glutamine; CellGro, Herndon, VA) with 10\% fetal bovine serum (FBS; Life Technologies, Inc. Carlsbad, Ca) and supplemented with 100 I.U./ml of penicillin and $100 \mu \mathrm{g} / \mathrm{ml}$ of streptomycin (ATCC, Inc. Manassas, VA). All cells were grown at 37C at 5\% CO2. For all EGF stimulations, cells were washed in 1x PBS (CellGro), and maintained in serum-free media (CellGro) for $1 \mathrm{hr}$ before experimentation. 


\section{Plasmids:}

TOM1L1 cDNA construct pDNR-LIB was purchased from (Plasmid 4612157

Dharmacon. Inc), cDNA constructs for knocksideways silencing, pMito-mCherry-FRB (henceforth, MitoTrap) or GFP-FKBP-CLCa were kindly provided by S. Royle (University of Warwick). The FDLP449AAAA hence CBM pD-HA-neo and Y460F hence SPM pD-HA-neo were kindly provided by W. Hong (Cancer and Developmental Cell Biology Division, Institute of Molecular and Cell Biology, Singapore).

\section{DNA plasmid preparation:}

Plasmids were heat shock transformed into DH5 $\alpha$ competent E. coli and selected for using antibiotics (chloramphenicol to select for wt-TOM1L1 pDNR-LIB; kanamycin to select for peGFP-C1, Clathrin binding mutant (CBM or FDPL ${ }^{449}$ AAAA) and Src phosphorylation mutant (SPM or Y460F). Plasmids were prepared using Sigma Aldrich midi kits (Sigma Aldrich, St Louis, MO) or Qiagen mini kits (Qiagen, Valencia, CA) and transfected via Polyjet (Froggabio). Plasmids were sent out for sequencing confirmation (TCAG, Sick Kids, Toronto, On). CBM ${ }^{446}{ }^{F D P L}{ }^{449}$ sequence, mutated to FDPL ${ }^{449}$ AAAA and Src phosphorylation mutant Y460F (Liu et al., 2009).

\section{Transfections:}

In order to prepare for DNA plasmid transfection, cells were washed once in 1x PBS, and replaced with $2 \mathrm{ml}$ of serum free DMEM. Polyjet (Froggabio) and cDNA was mixed in

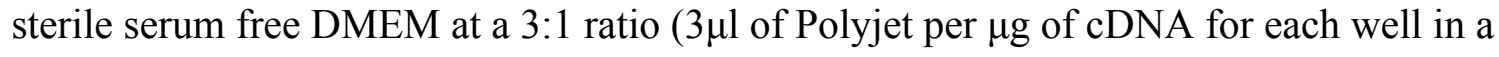
6-well plate). cDNA mixture was then pipetted 20 times and allowed to sit at room 
temperature for 20min before pipetting the cDNA mixture dropwise into each well of a 6well plate. siRNA transfection sequences and protocol used in knockdown experiments: TOM1L1 small interfering RNA (siRNA) duplex (human TOM1L1, sense GUGAGAAACUGAAUGUAUUtt; Dharmacon, Lafayette, CO) and siCON7 a nonspecific, scrambled siRNA duplex used as a control in knockdown experiments (human, sense CGUACUGCUUGCGAUACGGUU; Dharmacon, Lafayette, CO), were transfected with Lipofectamine RNAiMax (Life Technologies, Carlsbad, CA) at $220 \mathrm{pmol} / \mathrm{L}$ for RPE-wt (40-50\% confluency to start in a 12-well plate). Cells were then washed 3x in PBS and replaced with $0.9 \mathrm{ml}$ of Opti-Mem (CellGro) and 100ul of siRNA mixture was then added dropwise to each well and incubated for 3 to $4 \mathrm{hrs}$. Cells were then washed in 1x PBS and then replaced with DMEM containing 10\% FBS. RPE-wt cells were treated a second time after $24 \mathrm{hrs}$ and lysed after $72 \mathrm{hrs}$, following serum starvation (1 hr) and EGF stimulation (5ng/ml for 5, 10 and $15 \mathrm{~min})$.

\section{Cell lysis and immunoblotting:}

Whole cell lysates were prepared using a 2x Laemmli Sample Buffer stock (LSB, 0.5M

Tris $\mathrm{pH}$ 6.8, Glycerol, 10\% SDS) supplemented with a protease and phosphatase inhibitor cocktail (1mM sodium orthovanadate, 10nM okadaic acid, and 20nM Protease inhibitor cocktail) when blotting for phospho-proteins. Following EGF stimulation, cells were washed once with ice-cold PBS and aspirated. LSB was then added to each well $(150 \mathrm{uL}$, 12-well plate, 300ul, 6-well plate), scraped and transferred to an eppendorf. Lysates were then heated at $65 \mathrm{C}$ for $15 \mathrm{~min}$ and passed through a 27.5 gauge syringe. Reducing agents (10\% $\beta$-mercaptoethanol, and 5\% Bromophenol blue) were added following 
measurements of protein concentration using a BCA protein assay (Pierce, Fisher

Scientific, Waltham, MA). SDS PAGE (60V, 30min; followed by 120V, 90min), followed by transfer $(90 \mathrm{~V}, 2 \mathrm{hr}$ ) onto a PVDF membrane with a pore size of $0.45 \mu \mathrm{m}$ and binding capacity of 150-160 $\mu \mathrm{g} / \mathrm{cm}^{2}$ (BioRad, Mississauga, On). Membranes were then washed one in immunoblot wash (Tris-Base, $\mathrm{NaCl}$, Tween-20, and NP40), and incubated at room temperature in blocking solution (3\% BSA) for $1 \mathrm{hr}$. Primary antibodies were prepared by adding $1 \%$ BSA, $0.02 \%$ Sodium Azide and 1/1000 of antibody stock in immunoblot wash. BIORAD ChemiDoc XRS+ system was used for detection.

\section{Microscopy and image analysis:}

Total Internal Reflection microscopy: Samples were visualized using an Olympus IX81 TIRF microscope (IX81; Olympus, Inc,; Richmond Hill, On). TIRF microscopy was carried out using a 150x oil immersion objective lens with a numerical aperture of 1.45. Images were captured using a Hamamatsu C9100-13 EM-CCD, Li2CAM iCCD (w/GenIII GaAs intensifier) and rendered using Perkin Elmer Volocity software. Laser lines used were 491nm (GFP) and 561nm (RFP/A555), and two single-band bandpass filters were used 520/35, and 624/40. TIRF-Analysis: Coated pits as well as Gab1 and TOM1L1 punctate structure were detected by software developed in Matlab (Mathworks, Matick, MA), as described in (Aguet et al., 2013). Briefly, clathrin-coated pits that are diffraction limited were detected using a Gaussian-based model. Fluorescence intensity of proteins conjugated to red or green fluorophores were determined by the amplitude of the Gaussian model. 
Spinning Disc confocal microscopy: Spinning disc confocal images were obtained using a Nikon Olympus IX81 quipped with a Yokogawa CSU X1 scanhead and a 100x (NA 1.4) oil objective using a Hamamatsu C9100-13 EM-CCD camera. Excitation light was provided by $488 \mathrm{~nm}(100 \mathrm{mV}), 561(50 \mathrm{~mW})$ and $639 \mathrm{~nm}(40 \mathrm{mV})$ laser illumination, and emitted light was collected following passage through 525/50, 595/60 and 700/75 emission filters, as appropriate. Analysis was carried out as explained for TIRF microscopy. Detected objects were sorted as EGFR-positive or EGFR-negative based on the detection of A555-EGF within these objects. pGab1 signaling intensity within each detected AP-2 object was determined by the amplitude of the Gaussian model of pGab1 fluorescence within each structure (Aguet et al., 2013). Epifluorescence microscopy: Widefield epifluorescence microscopes (Axiovert 200M; Carl Zeiss, Inc.; Thornwood, NY), Epifluorescence microscopy was carried out using a 100x oil immersion objective lens with a numerical aperture of 1.45 on the Zeiss microscope, and $63 \mathrm{X}$ objective and 1.49NA on the Leica. Images were captured using a Hamamatsu Flash 4.0 and rendered using Perkin Elmer Volocity software. Excitation filters chosen were $340 / 25$ for DAPI, 481/15 for GFP, and 531/40 for RFP/Cy3, emission filters chosen were 480,40 for DAPI, 520/35 for GFP, and 610/60 for RFP/Cy3. Widefield epifluorescence image analysis was carried out using ImageJ software (National Institutes of Health, Bethesda, MA). Briefly, EGF-stimulated gain in intensity was calculated as the difference in mean fluorescence between EGF-stimulated cells and basal (unstimulated cells) of the same condition.

\section{Immunofluorescence Staining:}


The cells were grown on coverslips, serum starved for $1 \mathrm{hr}$. The media from the cells was aspirated and $1 \mathrm{ml}$ of 4\% Paraformaldehyde (PFA) fixing agent was added immediately to each well. The cells were then placed in the dark and incubated at room temperature for 20-30 minutes. Next the fixative was aspirated and cells were washed once with $2 \mathrm{~mL}$ of $100 \mathrm{mM}$ glycine solution (quenching solution). $2 \mathrm{ml}$ of glycine $+\mathrm{TX}-100$ (100 mM glycine $+0.1 \%$ Triton X-100 solution in PBS) permeabilization solution was then added to each well and incubated for 10 minutes. The cells were then washed 2-3 times with $1 \mathrm{X}$ PBS. $2 \mathrm{ml}$ of blocking solution (3\% BSA in 1X PBS) was then added and incubated in the dark for 20 minutes at room temperature. The cells were then washed with $1 \mathrm{X}$ PBS and labeled with primary antibody (pAkt S473, p-Gab1 pY627 and anti-AP2) was added and the cells were incubated in dark for 1 hour. The coverslips were then washed 8-10 times while shaking with $1 \mathrm{X}$ PBS. Following labeling with primary antibody, cells were stained with the appropriate fluorescently-conjugated secondary antibodies and then mounted on glass slides in fluorescent mounting media (Dako, Carpinteria, CA) or retained within aqueous media for imaging by TIRF microscopy.

\section{Knocksideways Silencing:}

RPE-wt cells (cells not stably expressing any exogenous fluorescent proteins) were transfected with cDNAs encoding pMito-mCherry-FRB (MitoTrap) or GFP-FKBPCLCa. On the day of the experiment, cells were treated with $1 \mu \mathrm{M}$ rapamycin for $10 \mathrm{~min}$, then were stimulated with $5 \mathrm{ng} / \mathrm{mL}$ EGF for $5 \mathrm{~min}$, or left unstimulated (basal). Cells were then immediately fixed and processed as described in Immunofluorescence staining. 


\section{Seamless Cloning (TOM1L1-GFP):}

Seamless cloning was used to fuse GFP to wt-TOM1L1. TOM1L1 was inserted into a peGFP-C1 vector. The primers were as follows:

L-Insert

5' - CGA GCT GTA CAA GGG ACT CAG ATC TAG ATC ACA GAT GGC GTT

TGG - 3'

L-Vector

5'- CCA AAC GCC ATC TGT GAT CTA GAT CTG AGT CCC TTG TAC AGC TCG $3^{\prime}$

R Vector:

5'- C TTA AGT AAA TAA TAG AGC TTC GAA TTC TGC -3'

R Insert:

5'- GCA GAA TTC GAA GCT CTA TTA TTT ACT TAA G -3'

The cDNA's had a concentration of $100 \mathrm{ng} / \mathrm{ml}$ and primers were used at a concentration of $10 \mu \mathrm{M}$. A master mix was made with the following volumes:

H2O 80uL, 10x PFU buffer 10uL, DMSO 5uL, 10mM dNTP $2.0 \mathrm{uL}$, PFU AD $2.0 \mathrm{uL}$ (Phusion High Fidelity Polymerase), Primers 2.0 uL, DNA 2.0 uL (wt-TOM1L1 \& eGFP). The master mix was split into 4 tubes and subjected to a gradient PCR. The conditions for the PCR gradient were set as follows:
i) $95^{\circ} \mathrm{C}$ for $2 \mathrm{~min}$
ii) $95^{\circ} \mathrm{C}$ for $30 \mathrm{sec}$
iii) $40-64{ }^{\circ} \mathrm{C}$ (gradient) for $1 \mathrm{~min}$
iv) $68^{\circ} \mathrm{C}$ for $12 \mathrm{~min}$ 
v) Repeat ii-iv 17 more times

vi) $4{ }^{\circ} \mathrm{C}$ until ready to use

The PCR products were resolved by agarose gel electrophoresis. Subsequently

approximately 1:1 molar ratio of insert and vector PCR reactions were made into a $20 \mathrm{uL}$ volume for recombination and incubated for $3-4 \mathrm{~h}$ with $1.0 \mu \mathrm{L}$ of $\mathrm{DnpI}$ (to digest methylated cDNA, which removed original PCR template). Following this the product was transformed into the DH5- $\alpha$ competent E.coli cells and colonies were selected after plating using the selection marker kanamycin. The final product was confirmed by sequencing and transfection into RPE-wt cells followed by immunoblotting.

\section{Agarose gel electrophoresis:}

$1 \%$ agarose gel was prepared by adding $0.6 \mathrm{~g}$ of agarose to $60 \mathrm{ml}$ of TAE buffer. The mixture was heated to dissolve the agarose and $3 \mu l$ of redsafe nucleic acid stain was added, in order to visualize the DNA. $2 \mu 1$ of the PCR product along with $2 \mu 1$ of loading dye was loaded on to the gel and subjected to electrophoresis at $110 \mathrm{~V}$.

\section{qRT-PCR:}

Following siRNA transfection ( $\operatorname{siCON7~\& ~siTOM1L1)~(72~hrs)~cells~were~subjected~to~}$ RNA extraction using RNA isolation kit (K0731-Thermo scientific). RNA concentration was measured using a spectrophotometer and RNA was diluted to a final concentration of $2 \mu \mathrm{g} / \mathrm{mL}$ for the reverse transcription (RT) reaction. RT-PCR was carried out using the RT PCR kit (superscript vilo-invitrogen -11754-050), with the following conditions (Run: $25^{\circ} \mathrm{C}$ for $10 \mathrm{~min}, 42^{\circ} \mathrm{C}$ for $60 \mathrm{~min}, 85^{\circ} \mathrm{C}$ for $5 \mathrm{~min}$ ). Following RT the cDNA was 
subjected to q-PCR using the qPCR mastermix: (TaqMan gene expression master mix, applied biosystems) and taq-man gene expression primers TOM1L1 and GAPDH (taqman primers, Life technologies). The reaction was run in a 96 well q-PCR plate in StepONE plus real time PCR (Applied Biosystems) with the following conditions (Run: $50^{\circ} \mathrm{C}$ for $2 \mathrm{~min}, 95^{\circ} \mathrm{C}$ for $10 \mathrm{~min},\left(95^{\circ} \mathrm{C}\right.$ for 15 seconds, $60^{\circ} \mathrm{C}$ for 60 seconds) $\mathrm{x} 40$ cycles). The data generated was analyzed using StepOne software v2.2.2 using the comparative $\mathrm{CT}$ method $(\Delta \Delta \mathrm{Ct})$ of analysis. 


\section{Results:}

Given the limitations of perturbation of clathrin by siRNA gene silencing and pharmacological approaches, confirming the requirement for clathrin in EGFR signaling using an additional, acute and specific method to perturb clathrin function was required. Using knocksideways silencing I further anchored the role of clathrin in early EGFR signaling leading to Akt activation. Furthermore, I demonstrated that the phosphorylated (and thus active) form of a key signaling intermediate in the EGFR signaling pathway, Gab1 (pGab1), is enriched within clathrin structures at the plasma membrane upon EGF stimulation, consistent with the interpretation that clathrin-coated pits at the plasma membrane are unique signaling platforms. I further examined how clathrin might function to facilitate EGFR signaling, by study of the clathrin-binding signaling adaptor protein TOM1L1. I found that TOM1L1 is present within plasma membrane clathrincoated pits and that it is required for EGFR signaling that leads to Akt activation. Collectively, these results indicate that TOM1L1 functions with clathrin to control EGFR signaling at the plasma membrane.

\section{Expression of GFP-FKBP-CLCa and mitoTrap effectively relocalizes CHC to the mitochondria.}

CLC with $\mathrm{N}$ or C-terminal fusion proteins, when overexpressed stably binds clathrin heavy chain $(\mathrm{CHC})$ approximately with a 1:1 stoichiometry and does not affect clathrin function, characterized by normal endocytosis demonstrated by normal transferrin (Tfn) uptake (Aguet et al., 2013). CLC and CHC form a stable interaction, evident from the fact the CLC can be co-immunoprecipitated with $\mathrm{CHC}$ when expressed 
in cells (Aguet et al., 2013). Moreover, overexpression of CLC constructs not only has little effect on clathrin function, but also displaces the endogenous, unlabelled CLC; expression of the endogenous CLC is drastically reduced upon overexpression of GFPtagged CLC (Auget et al. 2013). In light of these observations rerouting CLC to the mitochondria will force $\mathrm{CHC}$ to follow as well, thus making both CLC and CHC unavailable for their endogenous functions elsewhere in the cell, thus achieving the acute and specific inhibition of clathrin function required. RPE cells were co-transfected with GFP-FKBP-CLCa and pMito-mCherry-FRB (mitoTrap), treated or not with rapamycin, and then stained for $\mathrm{CHC}$ by immunofluorescence using an anti-CHC antibody. In the absence of rapamycin, both GFP-CLC and CHC exhibited normal localization, as seen by the fact that both signals exhibited strongly overlapping punctate distribution throughout the cell and concentration within the perinuclear region, consistent with the well described localization of clathrin in CCPs in the PM and on the trans-golgi network (TGN). Upon addition of rapamycin, both $\mathrm{CLC}$ and $\mathrm{CHC}$ redistributed to mitochondria, as seen by loss of punctate and perinuclear signal and strong overlap with MitoTrap (mitochondrial) fluorescence (Figure 10). Indeed it is difficult to find any punctate, peripheral structures in rapamycin-treated cells, indicating that the formation of CCPs has been effectively ablated under these conditions. These results show that knocksideways silencing of CLC is an effective way of sequestering $\mathrm{CHC}$ at the mitochondria, thereby inhibiting clathrin-dependent functions elsewhere in the cell. 


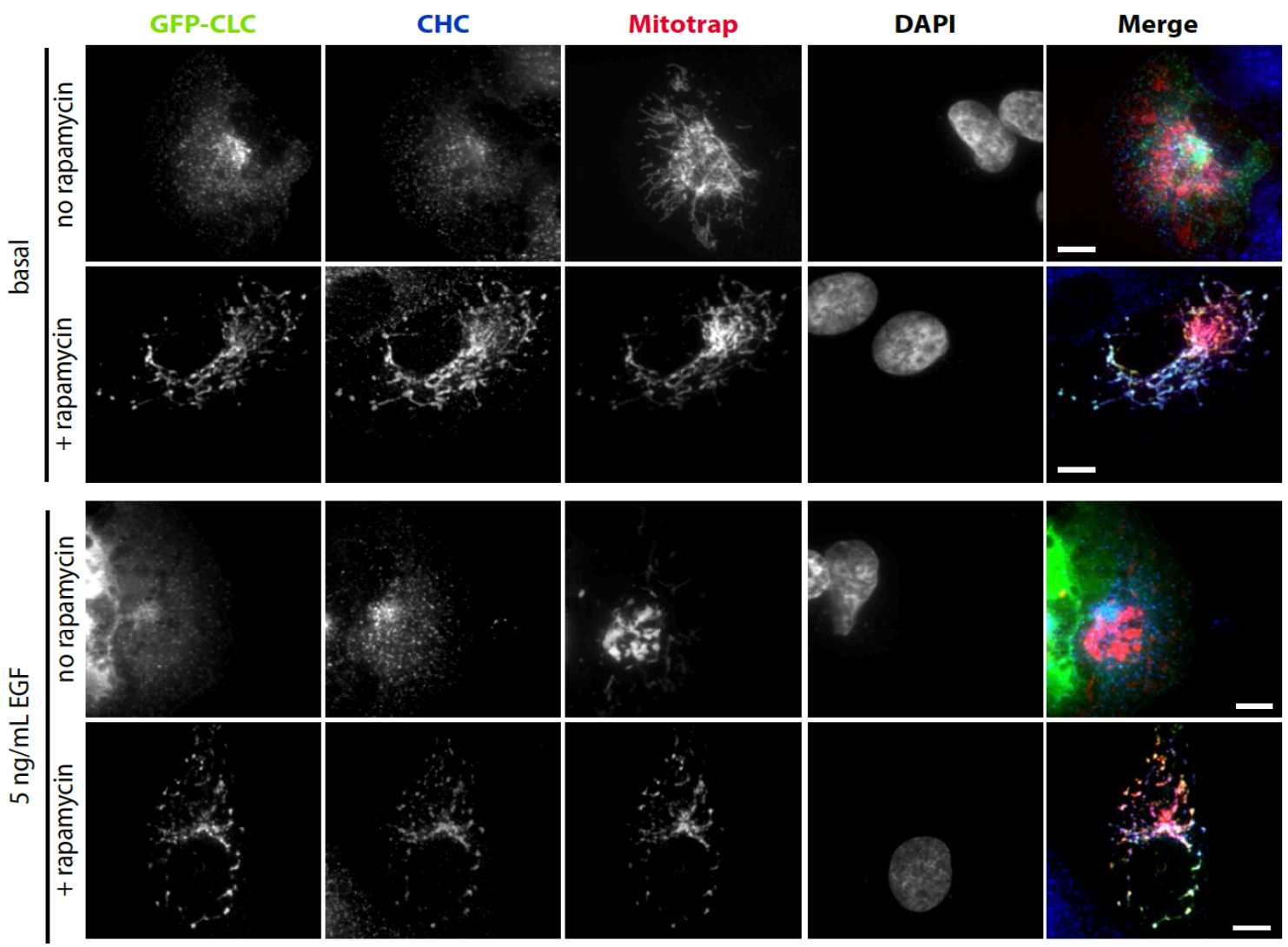

Figure 10: Expression of pMito-mCherry-FRB and GFP-FKBP-CLCa effectively relocalizes CHC to mitochondria upon rapamycin addition in RPE cells. RPE cells were co-transfected with cDNA encoding MitoTrap (pMito-mCherry-FRB) and GFP-FKBPCLCa. Following transfection, cells were treated with 1 $\mu \mathrm{m}$ rapamycin for $10 \mathrm{~min}$ and/or $5 \mathrm{ng} / \mathrm{mL}$ EGF for $5 \mathrm{~min}$, as indicated. Following treatment, cells were subjected to fixation and immunofluorescence labeling with anti-CHC antibodies and then imaging by spinning-disc confocal microscopy. Shown are representative sum projection micrographs, scale $=20 \mu \mathrm{m}$, showing effective relocalization of endogenous $\mathrm{CHC}$ to mitochondria in cells expressing both pMitomCherry-FRB and GFP-FKBP-CLCa, upon addition of rapamycin (Figure from Garay C, Judge G, Lucarelli S, Bautista S, Pandey R, Singh T, Antonescu CN. 2015. MBoC, in press).

\section{Knocksideways silencing of clathrin light chain (CLC) reduces EGF stimulated Akt phosphorylation.}

In order to determine if knocksideways silencing of CLC impairs EGF stimulated Akt phosphorylation, RPE cells were co-transfected with cDNAs encoding GFP-FKBPCLCa and pMito-mCherry-FRB (mitoTrap). Following transfection Akt phosphorylation was measured in individual cells by immunofluorescence staining using an anti-phosphoAkt s473 antibody (Figure 11A). Upon treatment with rapamycin, cells that were doubly 
transfected with GFP-FKBP-CLCa and mitoTrap displayed about $42.6 \pm 14.1 \%$

reduction in EGF stimulated Akt phosphorylation as compared to control (not expressing the GFP-FKBP-CLCa and MitoTrap) rapamycin treated cells $(n=3, p<0.05)$ (Figure

11B). Together with siRNA gene silencing and pitstop inhibition of clathrin (Garay $\mathrm{C}$ and Antgonescu CN, unpublished obsevrations, see Figure $3 \& 4$ ), these results establish that clathrin is selectively and specifically required for EGF-stimulated Akt phosphorylation.
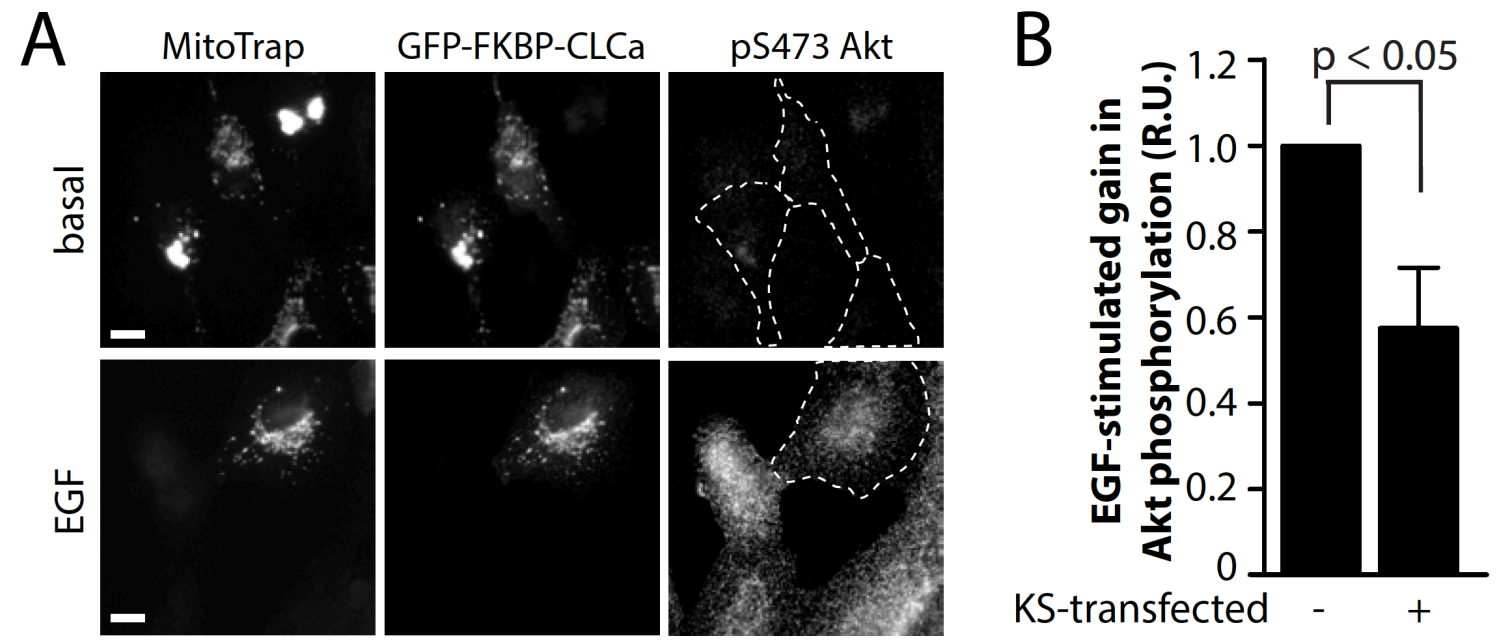

Figure 11. Knocksideways silencing of clathrin light chain reduces EGF-stimulated Akt phosphorylation in RPE cells. RPE cells were co-transfected with cDNA encoding mitoTrap (pMito-mCherry-FRB) or GFPFKBP-CLCa, after which cells were treated with $1 \mu \mathrm{M}$ rapamycin for $10 \mathrm{~min}$, then stimulated with 5 $\mathrm{ng} / \mathrm{mL}$ EGF for an additional $5 \mathrm{~min}$ in the continued presence of rapamycin. Following this, cells were fixed and processed for immunofluorescence staining using anti-phospho-Akt (pS473) specific antibodies. (A) Shown are representative micrographs, scale bar $=20 \mu \mathrm{m}$. (B) The mean fluorescence intensity of phospho-Akt (pS473) staining was quantified in cells expressing both mitoTrap and GFP-FKBP-CLCa (KS transfected + ) or only one of these exogenous fluorescent proteins (KS transfected - ). Shown are the mean $\pm \mathrm{SE}$ of the EGF-stimulated gain in phosphoAkt (pS473) levels $(\mathrm{n}=3) .{ }^{*}, \mathrm{p}<0.05$. (Figure from Garay $\mathrm{C}$, Judge G, Lucarelli S, Bautista S, Pandey R, Singh T, Antonescu CN. 2015. MBoC, in press). 
Expression of GFP-FKBP-CLCa and mitoTrap is of no effect on EGF stimulated Akt phosphorylation in the absence of rapamycin.

In order to determine if the inhibition of EGF-stimulated Akt phosphorylation was specific to the sequestration of clathrin to mitochondria upon addition of rapamycin, I examined EGF-stimulated Akt phosphorylation in cells expressing the clathrin knocksideways constructs in the absence of rapamycin. RPE-wt cells were co-transfected with cDNAs encoding GFP-FKBP-CLCa and mitoTrap. Following transfection Akt phosphorylation was measured in individual cells by immunofluorescence staining using an anti-phospho-Akt s473 antibody (Figure 12A). Importantly, the expression of these constructs had no detectable effect on EGF stimulated Akt phosphorylation in the absence of rapamycin (Figure 12A\&B) i.e. under conditions where CLC/CHC were not sequestered to the mitochondria. Consistent with previous observations that expression of GFP-CLC does not impact clathrin function for endocytosis, overexpression of fluorescently-tagged CLC also did not impact EGF-simulated Akt phosphorylation, suggesting that this strategy can be used to selectively study the effect of acute clathrin depletion on EGFR signaling. 
A

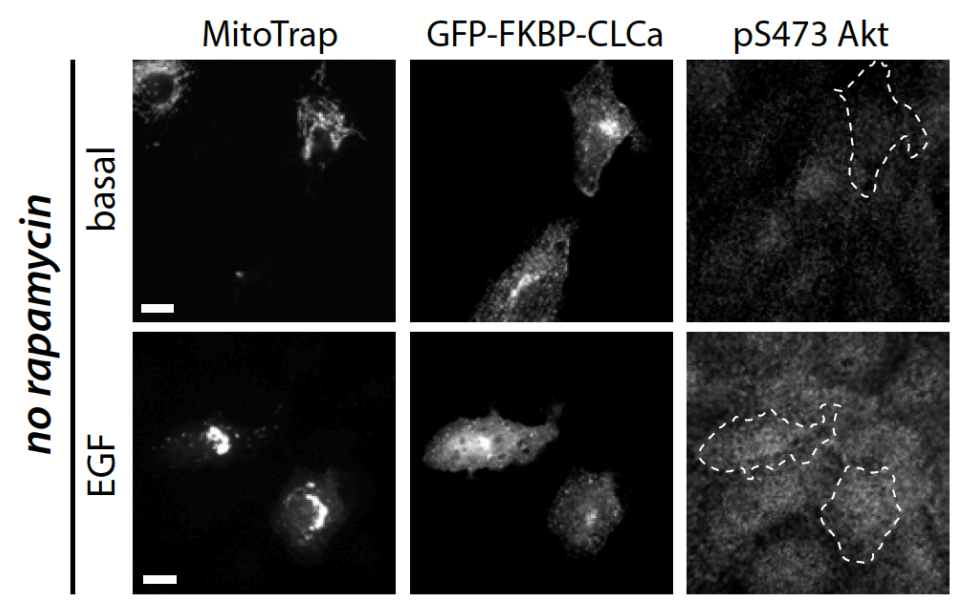

B

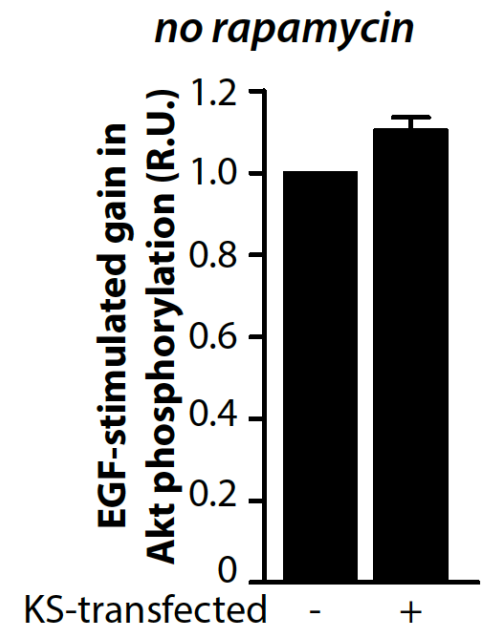

Figure 12: Expression of pMito-mCherry-FRB and GFP-FKBP-CLCa does not impact EGF-simulated Akt phosphorylation in the absence of rapamycin. RPE cells were co-transfected with cDNA encoding MitoTrap (pMito-mCherry-FRB) and GFP-FKBPCLCa. (A-B) Following transfection, cells were stimulated with $5 \mathrm{ng} / \mathrm{mL}$ EGF or left unstimulated (basal). Note that in contrast to the results shown in Figure 2, the experiments presented in (A-B) were performed in absence of rapamycin. Following treatment, cells were fixed and processed for immunofluorescence staining using anti-phospho-Akt (S473) specific antibodies. Shown in (A) are representative micrographs, scale bar $=20 \mu \mathrm{m}$. The mean fluorescence intensity of phosphoAkt (pS473) staining was quantified in cells expressing both MitoTrap and GFP-FKBP-CLCa cells (KS transfected + ) or only one of these exogenous fluorescent proteins (KS transfected -). Shown in (B) are the mean \pm SE of phospho-Akt (S473) levels $(n=3)$. (Figure from Garay C, Judge G, Lucarelli S, Bautista S, Pandey R, Singh T, Antonescu CN. 2015. MBoC, in press).

\section{p-Gab1 is recruited to Clathrin coated pits (CCPs) at the plasma membrane upon EGF stimulation.}

Thus far the results have indicated a role of clathrin early EGFR signaling leading to Akt phosphorylation. Since inhibition of dynamin (which allows formation of clathrin structures at the PM but impairs their ability to form intracellular vesicles) with the chemical inhibitor dynasore or siRNA gene silencing was without effect on EGFstimulated Akt phosphorylation (Figure 3, Introduction), clathrin likely functions at the plasma membrane, possibly as a scaffold for EGFR signaling intermediates. To determine if clathrin indeed functions as a signaling scaffold, I further examined whether Gab1, a key signaling intermediate leading to Akt phosphorylation, is recruited to CCPs upon activation following EGF stimulation. In order to do so, I performed 
immunofluorescence labeling of EGF stimulated and unstimulated cells expressing RFPCLC with antibodies that specifically label endogenous phosphorylated Gab1 (p-Gab1, Y627). Following fixation cells were immunostained, mounted and subjected to total internal reflection fluorescence microscopy (TIRF-M). In unstimulated (basal) cells, I observed a number of $\mathrm{p}-\mathrm{Gab} 1$ puncta, yet by visual inspection, these do not seem to overlap with CCPs (Figure 13A). The pGab1 signal detected by IF in basal cells may represent background antibody staining since not much phosphorylated Gab1 was detected by western blotting in this condition (Figure 3). However, in the stimulated (EGF) cells, a portion of p-Gab1 puncta overlap with CCPs as indicated by the arrows (Figure 13A). Although visual examination of fluorescent images can be informative, a systematic unbiased computational approach is required to determine if the recruitment of p-Gab1 to CCPs was indeed enhanced by EGF, and whether it was specific or instead resulted from random overlap of dense punctate objects in the image. The overlap between $\mathrm{p}-\mathrm{Gab} 1$ and clathrin was determined using automated analysis that involved detection of clathrin structures using Gaussian modeling of the point-spread function of diffraction-limited CCPs, followed by systematic quantification of p-Gab1 fluorescence within these detected objects (Aguet et al., 2013). Using this method, EGF stimulation elicited a 2.3-fold increase in pGab1 signal intensity within clathrin structures (Figure 13B).

To eliminate the possibility that the proportion of pGab1 signal detected within clathrin structures was due to random overlap of dense punctate structures in an image, a randomized image set was generated by shifting the pGab1 channel by at least 20 pixels, thereby scrambling the positions of the pGab1 signal relative to that of clathrin, while 
maintaining the same density of clathrin and pGab1 objects in the image. EGF-

stimulated and basal (unstimulated) cells exhibited similar p-Gab1 intensities upon

quantification of the scrambled images (Figure 13B). This confirms that the EGF-

stimulated enrichment of pGabl within clathrin structures is specific and selective, and

does not occur simply as a result of random overlap of dense punctate structures.

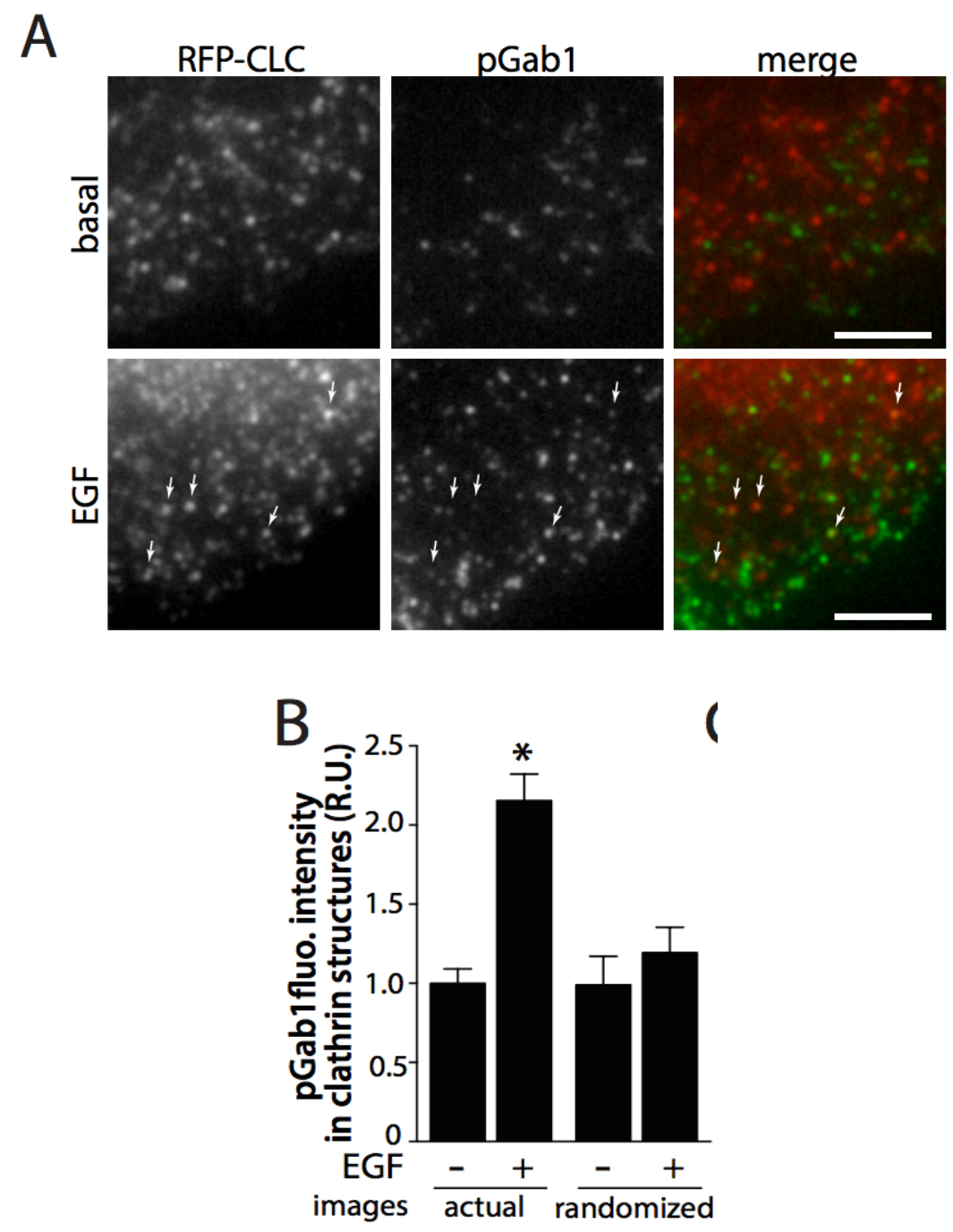

Figure 13: Phosphorylated Gab1 is enriched within plasma membrane clathrin structures upon EGF stimulation in RPE cells. RPE cells stably expressing Tag-RFP-T-CLC (RFP-CLC) were stimulated with $5 \mathrm{ng} / \mathrm{mL}$ EGF for 3 min or left unstimulated (basal), fixed and labeled with antibodies that specifically recognize Gab1 phosphorylated on Y627 (pGab1), followed by imaging using total internal reflection fluorescence microscopy (TIRF-M). (A) Shown are representative micrographs, scale bar $=5$ $\mu \mathrm{m}$. (B) Micrographs obtained by TIRF-M were subjected to automated detection of clathrin structures as 
in (Aguet et al., 2013), see Materials and Methods, followed by quantification of pGab1 and RFP-CLC in each detected object. Shown in (B) is the mean pGab1 fluorescence intensity detected within all clathrin structures in each image (actual image), as well as in images in which the pGab1 signal was randomized (see Materials and Methods) to determine the amount of pGab1 that occurs as a result of random overlap of clathrin and pGab1 puncta $(>10,000$ clathrin structures from $>45$ cells from 4 independent experiments, * $\mathrm{p}<0.05$ ). (Figure from Garay C, Judge G, Lucarelli S, Bautista S, Pandey R, Singh T, Antonescu CN. 2015. MBoC, in press).

\section{pGab1 is largely restricted to clathrin structures containing EGFR}

While pGab1 was found to be enriched in CCPs upon EGF stimulation, it was apparent that pGab1 signal was found only in a minority of CCPs. This is consistent with the fact that EGFR is only recruited to a fraction of CCPs (Garay et al, 2015, MBoC). Since Gab1 associates with EGFR via binding to Grb2, this suggests that pGab1 may be found predominantly within CCPs that harbor EGFR. In order to determine whether the localization of pGab1 was restricted to clathrin structures that also contained EGFR, I performed triple-labeling experiments in cells stimulated with fluorescently labelled EGF (A555-EGF, to label ligand-bound EGFR) for 3 min, followed by immunofluorescence labeling of the plasma membrane clathrin adaptor AP-2 as well as pGab1. Following the cells were subjected to spinning disc confocal microscopy. Consistent with results obtained by TIRF-M (Figure 14A), pGab1 was again observed in a subset of clathrin structures (Figure 14A). Automated detection of AP-2 structures in ventral sections ( $\mathrm{n}=$ 19) allowed classification of clathrin structures as "EGFR positive" (42.0 $\pm 1.8 \%)$ or "EGFR negative" $(58.0 \pm 1.8 \%)$, followed by quantification of pGab1 signal intensity within each of these cohorts. The EGFR-positive cohort of AP-2 structures exhibited a 
strong $(\sim 2 \mathrm{x})$ enrichment of $\mathrm{pGab} 1$ relative to those that were EGFR-negative (Figure 14B).

Even though immunofluorescence labeling of pGab1 displayed an expected gain in staining intensity upon EGF stimulation, there was significant background labeling observed in basal cells not expected to have much phosphorylated Gab1 (Figure 14C), as was observed in the images obtained by TIRF-M (Figure 13). To determine the proportion of pGab1 signal detected within AP-2 structures that was due to random overlap of punctate structures, a similar randomization of the pGab1 images relative to AP2 and A555-EGF images was performed as explained for pGab1 (Figure 13B). The pGab1 staining intensity within EGFR-positive AP-2 objects detected within the randomized pGab1 image set was similar to that of the EGFR-negative AP-2 structures in the actual (non-randomized) image sets (Figure 14B). Hence, the pGabl staining detected within EGFR-negative structures represents background, random overlap between pGab1 and AP-2 signals. Therefore, pGab1 was selectively enriched in clathrin structures containing EGFR. 

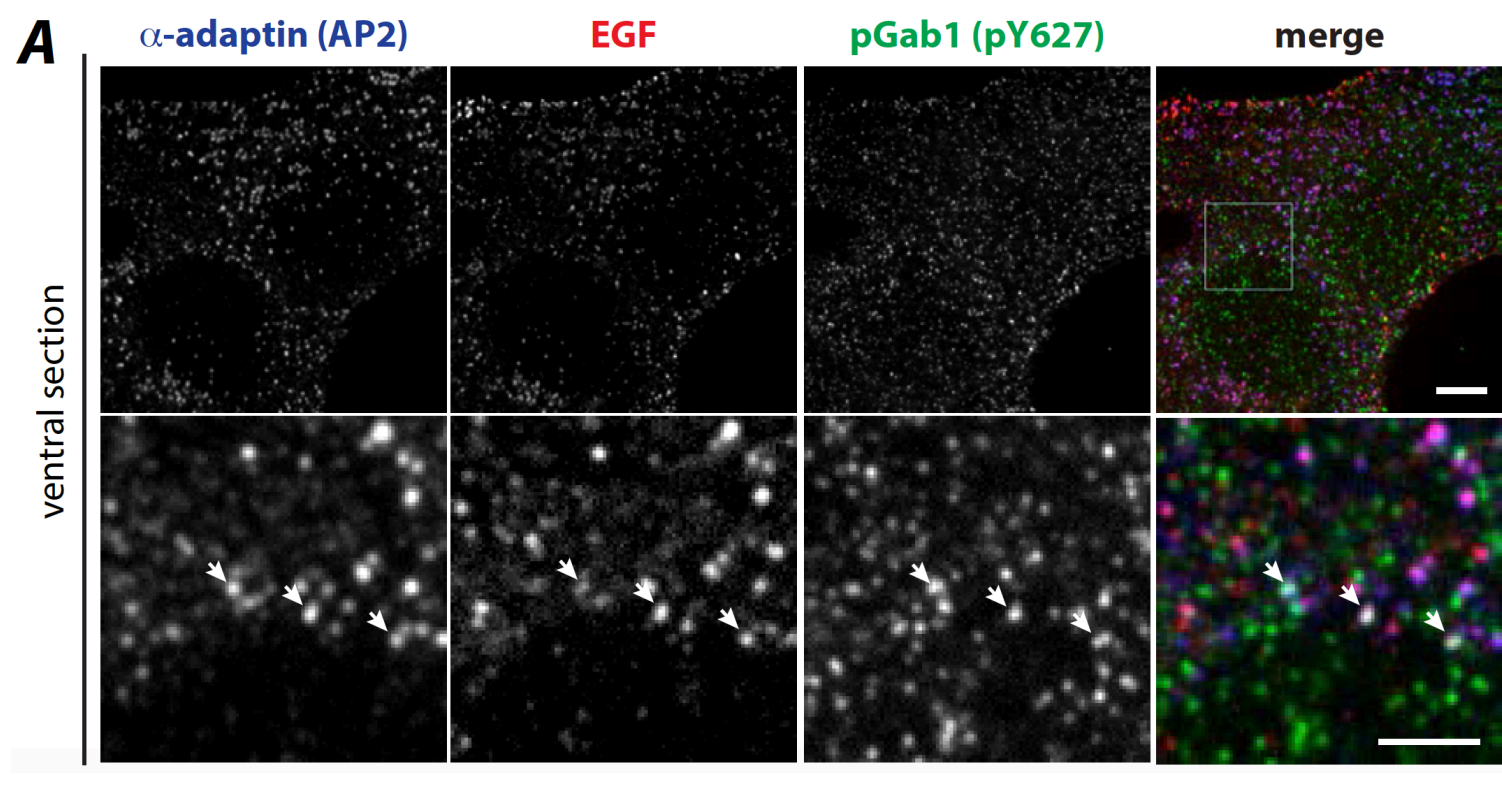

B
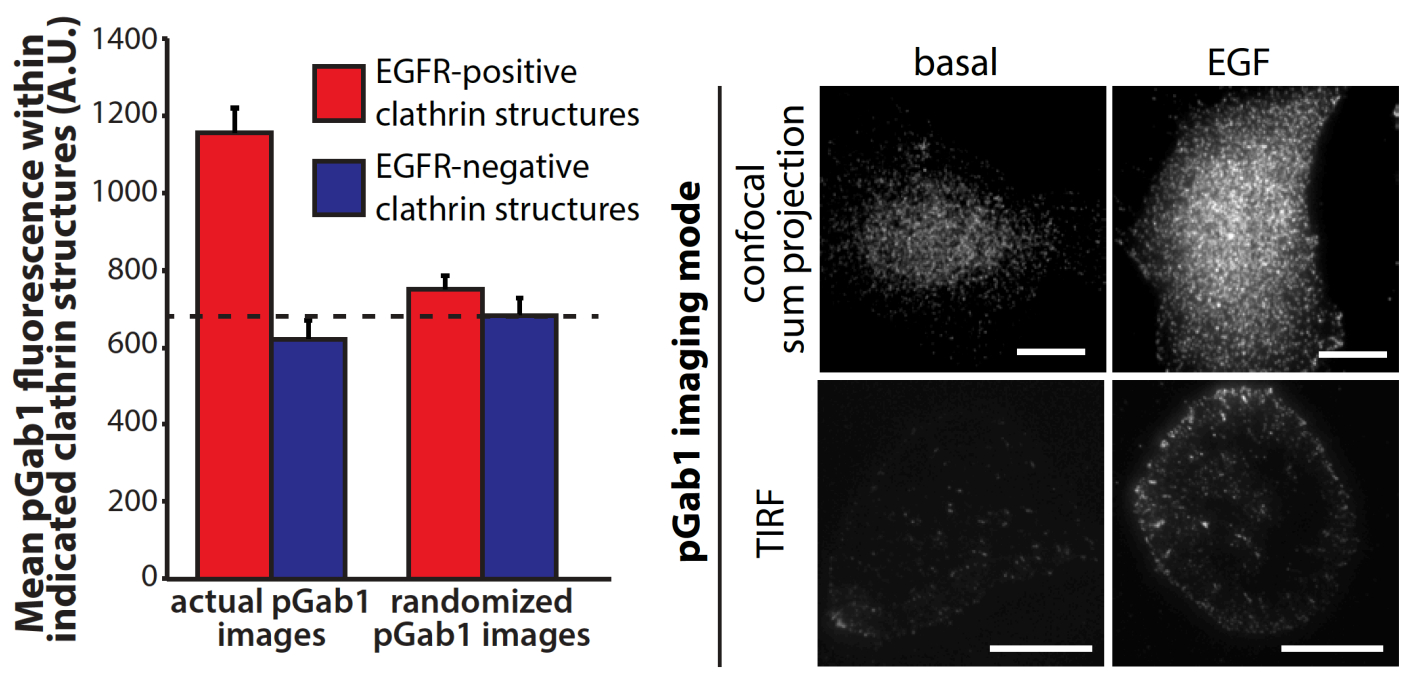

Figure 14: pGab1 is largely restricted to clathrin structures containing EGFR in RPE-wt cells. (A-B) Wildtype RPE cells were treated with $5 \mathrm{ng} / \mathrm{mL}$ EGF conjugated to Alexa-555 (EGF-A555) for 3 min and immediately fixed and subjected to immunofluorescence staining using anti-phospho-Gab1 (pY627) or anti- $\alpha$-adaptin antibodies (to label AP-2 and thus plasma membrane clathrin structures). Shown in (A) are representative fluorescence micrographs obtained by spinning disc confocal microscopy of a single zsection near the ventral surface of a cell, as indicated. Scale $=20 \mu \mathrm{m}$ (top panels) or $5 \mu \mathrm{m}$ (magnified inset of boxed region of top panel). AP-2 structures were subject to automated detection, sorted on the basis of EGFR content, followed by quantification of pGab1 levels, as described in Materials and Methods. Shown in (B) are the 3 means \pm SE of mean pGab1 intensity within EGFR-positive and EGFR-negative plasma membrane clathrin/AP-2 structures, as well as in images in which the pGab1 signal was scrambled to determine the amount of pGab1 that occurs as a result of random overlap of AP-2 and pGab1 puncta. The dashed line represents pGab1 fluorescence intensity that results from random overlap of pGab1 and clathrin/AP-2 signals. (C) Shown are representative fluorescence micrographs obtained following immunofluorescence labeling using pGab1 antibodies by spinning disc confocal microscopy (sum projection) or TIRF microscopy, as indicated, scale $=20 \mu \mathrm{m}$. (Figure from Garay C, Judge G, Lucarelli S, Bautista S, Pandey R, Singh T, Antonescu CN. 2015. MBoC, in press). 
Collectively, these results strongly support and establish a functional requirement for clathrin in signaling. Using three independent approaches, siRNA gene silencing, pharamacological inhibition and knocksideways silencing of clathrin, all result in reduced Akt phosphorylation upon EGF stimulation. This indicates that the requirement for clathrin is likely to enrich key signaling intermediates within clathrin structures, such as the enrichment of pGab1 within CCPs. These results also imply that the signaling molecule(s) required for the phosphorylation of Gab1 upon EGF stimulation amy also be enriched and recruited to CCPs. Next I examined whether the clathrin- and SFK-binding signaling adaptor TOM1L1 is required for EGF-stimulated Akt phosphorylation, and whether an interaction between TOM1L1 and clathrin underlie sthe role of TOM1L1 in regulating EGFR signaling at the plasma membrane.

\section{siRNA knockdown of TOM1L1 reduces EGF stimulated Akt phosphorylation.}

In order to determine knockdown efficiency of TOM1L1, I employed q-RT-PCR due to the lack of commercially available antibodies against TOM1L1. The mRNA transcripts levels were measured in siTOM1L1 treated cells and compared to control siRNA treated cells using a taq-man q-PCR reaction (see materials and methods). There was a significant reduction in relative mRNA levels of TOM1L1 $83.4 \pm 9.2 \%$ in the siTOM1L1 treated cells as compared to control ( $\mathrm{n}=3$ ) (Figure 15A).

In order to determine if TOM1L1 is required for early EGFR signaling, siRNA gene silencing was used. RPE cells were treated with siRNA targeting TOM1L1 or unrelated (control) siRNA. Cells were then stimulated with $5 \mathrm{ng} / \mathrm{mL}$ EGF for the indicated times (Figure 15B). Knockdown of TOM1L1 resulted in a significant reduction 
of Akt phosphorylation upon EGF stimulation as compared to the control $(\mathrm{n}=9, \mathrm{p}<0.05)$

\section{(Figure 15B\&C).}

A

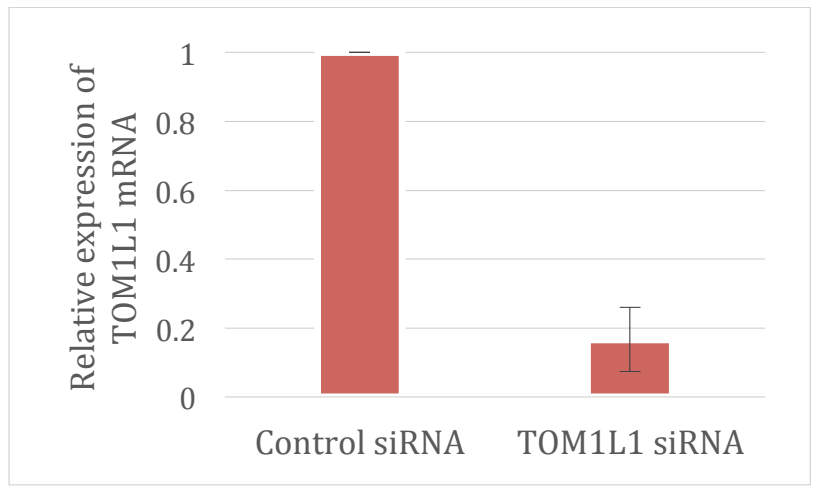

B

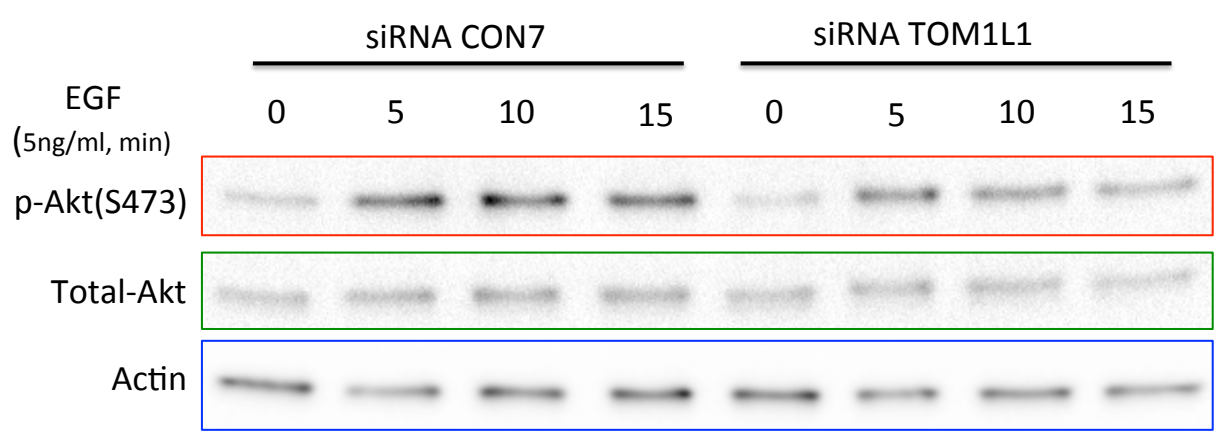




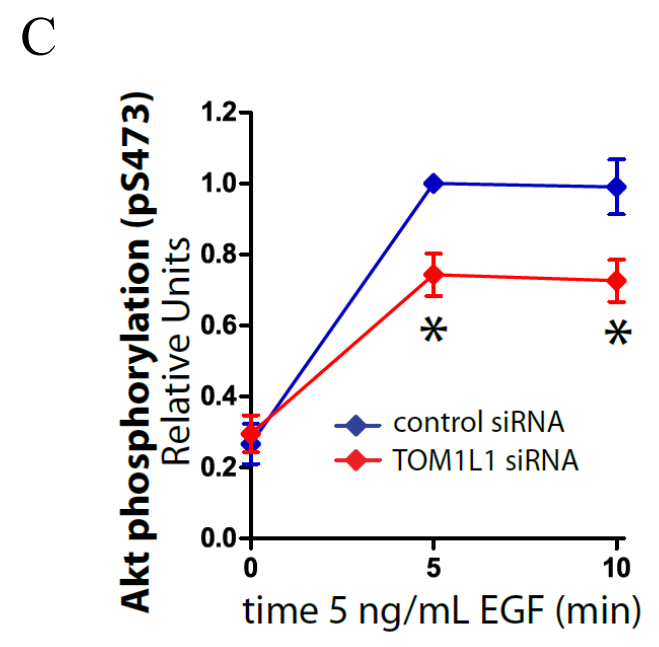

Figure 15: Silencing of TOM1L1 results in reduced Akt phosphorylation. RPE cells were treated with siRNA targeting TOM1L1 or unrelated (control) siRNA. (A) Quantification of TOM1L1 knockdown using qRT-PCR. Shown is the relative expression of TOM1L1 mRNA in TOM1L1 knockdown condition as compared to control siRNA. There was an $83.4 \pm 9.2 \%$ reduction in TOM1L1 mRNA levels as compared to control $(\mathrm{n}=3)$.Cells were then stimulated with $5 \mathrm{ng} / \mathrm{mL}$ EGF for the indicated times. (B) Shown are immunoblots of whole-cell lysates using antibodies recognizing the phosphorylated forms of Akt, total Akt and actin (loading control). (C) Also shown are the mean \pm SE of pS473-Akt values ( $\mathrm{n}=9$ ), ${ }^{*}, \mathrm{p}<0.05$, relative to control, EGF-stimulated condition.

\section{siRNA knockdown of TOM1L1 does not affect EGF stimulated MAPK and EGFR phosphorylation.}

TOM1L1 has been shown to control EGFR intracellular membrane traffic. Hence, it is possible that a reduction in EGF-stimulated Akt phosphorylation could be the result of less receptors at the cell surface and/or other defects in EGFR membrane traffic, or possibly other, broader regulation of EGFR. Indeed we previously demonstrated (Garay et al., 2015, In press) that siRNA gene silencing of CHC did not alter EGFR cell surface levels as demonstrated by a variety of methods including 1) antibody labeling of intact cells with anti-EGFR antibodies, 2) EGF ligand binding assay, 3) measurement of EGF- 
stimulated Erk and EGFR phosphorylation. This indicates that clathrin is functionally required for EGF-stimulated Gab1 and Akt phosphorylation, but not for EGFR autophosphorylation, or for activation of other signaling pathways such as Erk. If clathrin is required for EGFR signaling due to its ability to recruit TOM1L1, then it would be expected that TOM1L1 also selectively contributes to EGF-stimulated Akt phosphorylation, without having a role in EGFR phosphorylation or Erk pathway activation.

To determine if TOM1L1 altered EGF-stimulated Akt phosphorylation as a result of a reduction of EGFR cell surface levels or other non-selective changes in EGFR signaling, I examined the effect of TOM1L1 siRNA gene silencing on EGF-stimulated Erk and EGFR phosphorylation. siRNA gene silencing of TOM1L1 had no effect on EGF stimulated Erk and EGFR (Y1068) phosphorylation as compared to control (Figure 16). This indicated that TOM1L1 specifically plays a role in early EGFR signalling specifically leading to Akt phosphorylation and does not affect global EGFR signaling. Futhermore, these results suggest that the ability of TOM1L1 to control clathrindependent EGFR signaling may be related to the ability of TOM1L1 to bind clathrin; however, the localization of TOM1L1 to clathrin-coated pits at the plasma membrane has thus far not been demonstrated. 


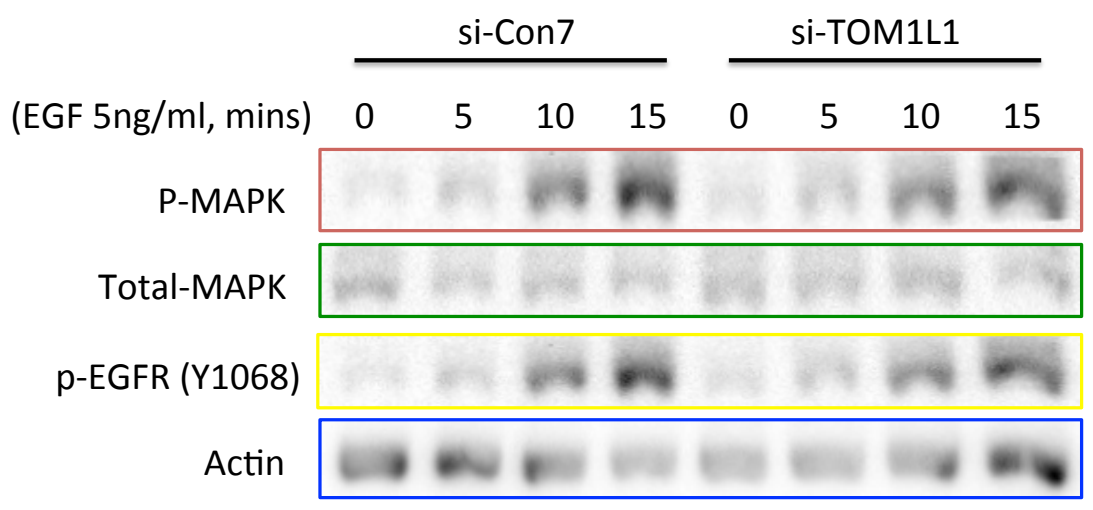

Figure 16: Silencing of TOM1L1 does not affect p42/44 MAPK phosphorylation. RPE cells were treated with siRNA targeting TOM1L1 or unrelated (control) siRNA. Cells were then stimulated with $5 \mathrm{ng} / \mathrm{mL}$ EGF for the indicated times. Shown are immunoblots of whole-cell lysates using antibodies recognizing the phosphorylated forms of p42/44 MAPK, total MAPK, EGFR (pY1068) and actin(loading control).

\section{Generation of wt-TOM1L1-GFP.}

In order to determine the cellular localization of TOM1L1, I generated a fusion protein by attaching green fluorescent protein to human TOM1L1 (TOM1L1-GFP). GFP was fused to the N-terminal of TOM1L1 using seamless cloning (see Materials and Methods). Following sequencing to ensure that the intended cDNA construct for expression of GFP-tagged TOM1L1 was indeed correctly generated, I confirmed that the expressed protein had the expected molecular weight by transfection into RPE cells, followed by western blotting of whole cell lysates with an anti-GFP antibody. The cell sample transfected with a GFP-TOM1L1 cDNA construct indeed exhibited a band of approx. $79 \mathrm{kDa}$, consistent with GFP-TOM1L1 (26 kDa for GFP fused to $\sim 53 \mathrm{kDa}$ for TOM1L1), which was not seen in cells transfected with a construct for expression of eGFP only or untransfected cells (Figure 17). The generation of TOM1L1-GFP served as a significant tool in determining TOM1L1 localization vis-à-vis CCPs with and without EGF stimulation. 


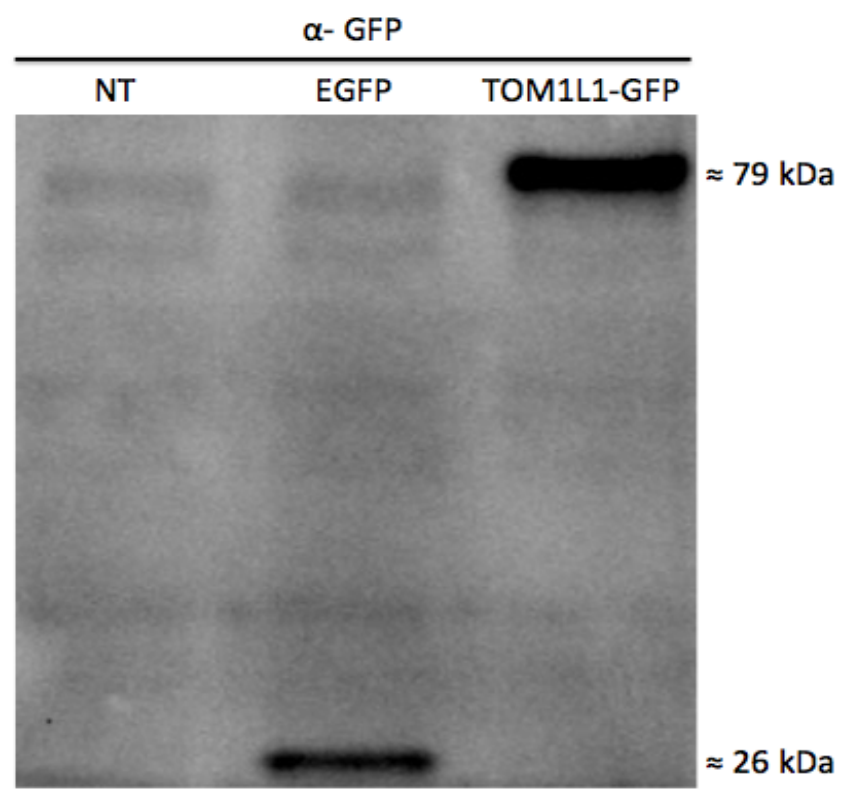

Figure 17: Expression of exogenous TOM1L1-GFP and EGFP in RPE cells. RPE cells were transfected with TOM1L1-GFP and EGFP. Non-transfected (NT) cells served as control. Shown is the immunoblot of whole-cell lysates using anti-GFP antibody.

\section{TOM1L1 is constitutively detected within CCPs.}

So far the results indicate a novel role for TOM1L1 in controlling early EGFR signaling, specifically EGF stimulated Akt phosphorylation at the cell surface. In order to determine if TOM1L1 may be doing this as a result of its recruitment to CCPs, I sought to determine whether TOM1L1 is present within CCPs at the plasma membrane, which had not been shown previously.

In order to determine the localization of TOM1L1 vis-à-vis CCPs, RPE cells stably expressing RFP-CLC were transfected with TOM1L1-GFP. Following transfection cells were stimulated with $5 \mathrm{ng} / \mathrm{ml}$ of EGF for indicated times or left unstimulated (basal), and then fixed and mounted for TIRF-M. For both basal and EGF stimulated condition significant amount of TOM1L1 was recruited to clathrin structures (Figure 18A). In 
order to quantify the extent of recruitment of TOM1L1-GFP to clathrin structures, I again used automated image analysis as had been done for pGab1 (Figure 13). Diffractionlimited clathrin structures were detected using Gaussian modeling of the point spread function (PSF) of CCPs, followed by systematic quantification of TOM1L1-GFP fluorescence within these detected objects (Aguet et al., 2013). (Figure 18B). The background levels of TOM1L1-GFP overlap with clathrin structures were determined by "scrambling" the TOM1L1-GFP channel by shifting the position of the TOM1L1-GFP image 20 pixels relative to the clathrin image, similarly to that done to obtain a scrambled set of p-Gab1 images (Figure 13). Quantification of these scrambled images revealed similar levels of background for all the conditions (Figure 18B) that was much lower than the signal detected in the actual (unscrambled) images, thus suggesting that the overlap between TOM1L1-GFP and CCPs is significant and above background.

TOM1L1 has been documented to bind clathrin, however its recruitment to the plasma membrane CCPs has never been demonstrated. 

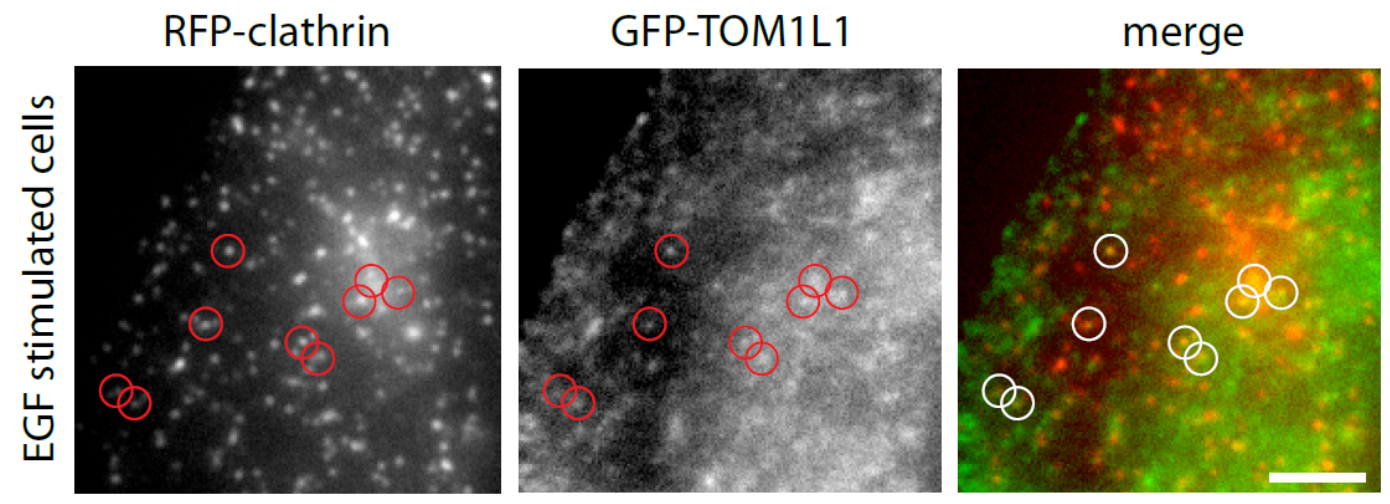

B

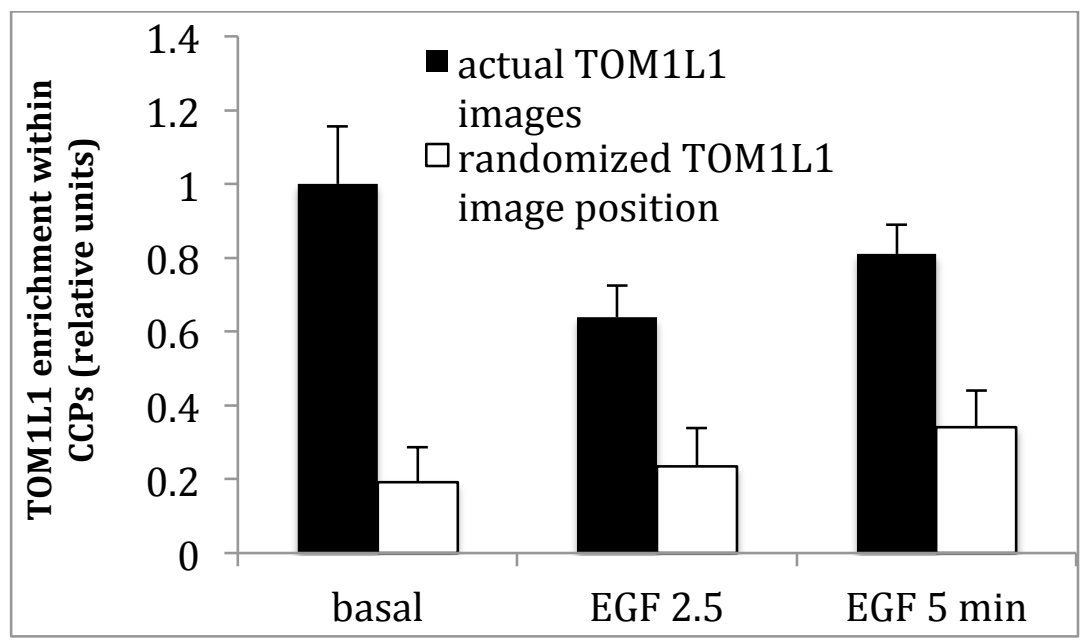

Figure 18: Recruitment of TOM1L1-GFP to CCPs. RPE cells stably expressing Tag-RFP-T-CLC (RFPCLC) were transfected with GFP fused to TOM1L1 (TOM1L1-GFP). Cells were then stimulated with 5 $\mathrm{ng} / \mathrm{mL}$ EGF for $2.5 \mathrm{~min}$ or left unstimulated (basal), then fixed and imaged using total internal reflection fluorescence microscopy (TIRF-M). (A) Shown are representative micrographs, scale bar $=5 \mathrm{~mm}$. Micrographs obtained by TIRF-M were subjected to automated detection of clathrin structures, followed by quantification of TOM1L1-GFP and RFP-CLC in each detected object. (B) Shown in is the mean TOM1L1-GFP fluorescence intensity detected within all clathrin structures in each image (actual image), as well as in images in which the TOM1L1-GFP signal was randomized (see Materials and Methods) to determine the amount of TOM1L1-GFP that occurs as a result of random overlap of clathrin and TOM1L1GFP puncta from 3 independent experiments, $\left.{ }^{*} \mathrm{p}<0.05\right)$.

\section{Expression of a clathrin binding-defective mutant of TOM1L1 affects basal and EGF- stimulated Akt phosphorylation.}

So far we established a role of TOM1L1 in early EGFR signaling leading to Akt activation. Importantly it does not affect global EGFR signaling, evident by the fact that 
TOM1L1 silencing is of no effect on EGF-stimulated Erk phosphorylation. Moreover, we provided the first evidence of TOM1L1 recruitment to clathrin-coated pits at the plasma membrane. I next sought to determine how each of the interactions of TOM111 (e.g. with clathrin) impact EGF-simulated Akt phosphorylation.

TOM1L1 interacts with clathrin heavy chain and Src family kinaes via separate interactions. TOM1L1 mutants defective in each of these interactions: 1) with clathrin via ${ }^{a}{ }^{446} \mathrm{FDPL}^{449}$ sequence, mutated to FDPL ${ }^{449} \mathrm{AAAA}$ (clathrin binding mutant, or CBM) and 2) with Src-family kinases (SFKs, e.g. c-Src): Y460F (henceforth Srcphosphorylation mutant, SPM) (Liu et al., 2009) were transfected into RPE-wt cells along with wt-TOM1L1-GFP and eGFP vector. Cells were then stimulated with $5 \mathrm{ng} / \mathrm{ml}$ of EGF for 5 minutes or left unstimulated (basal). Following transfection and EGF stimulation Akt phosphorylation was measured in individual cells by immunofluorescence staining using an anti-phospho-Akt s473 antibody (Figure 19A). Cells transfected with eGFP, wtTOML1-GFP and the TOM1L1-Y460F displayed a significant increase in EGF stimulated Akt phosphorylation as compared to basal, similar to what was observed in untransfected cells $(n=3, p<0.05)$ (Figure 19B). Interestingly, there was a robust increase in Akt phosphorylation in the basal condition for the TOM1L1-FDPL449AAA and the TOM1L1-Y460F. Importantly, while EGF stimulation further significantly enhanced the Akt phosphorylation in cells expressing TOM1L1-Y460F, cells expressing TOM1L1-FDPL449AAA did not exhibit an increase in Akt phosphorylation upon EGF stimulation (Figure 19B). These results demonstrate that the clathrin binding-deficient mutant of TOM1L1 acted as dominant negative and inhibited the gain in EGF stimulated Akt phosphorylation. In other words, the difference between basal and EGF stimulated 
Akt phosphorylation levels (delta- $\Delta$ ) was not significant as compared to other conditions

(Figure 19C)

A
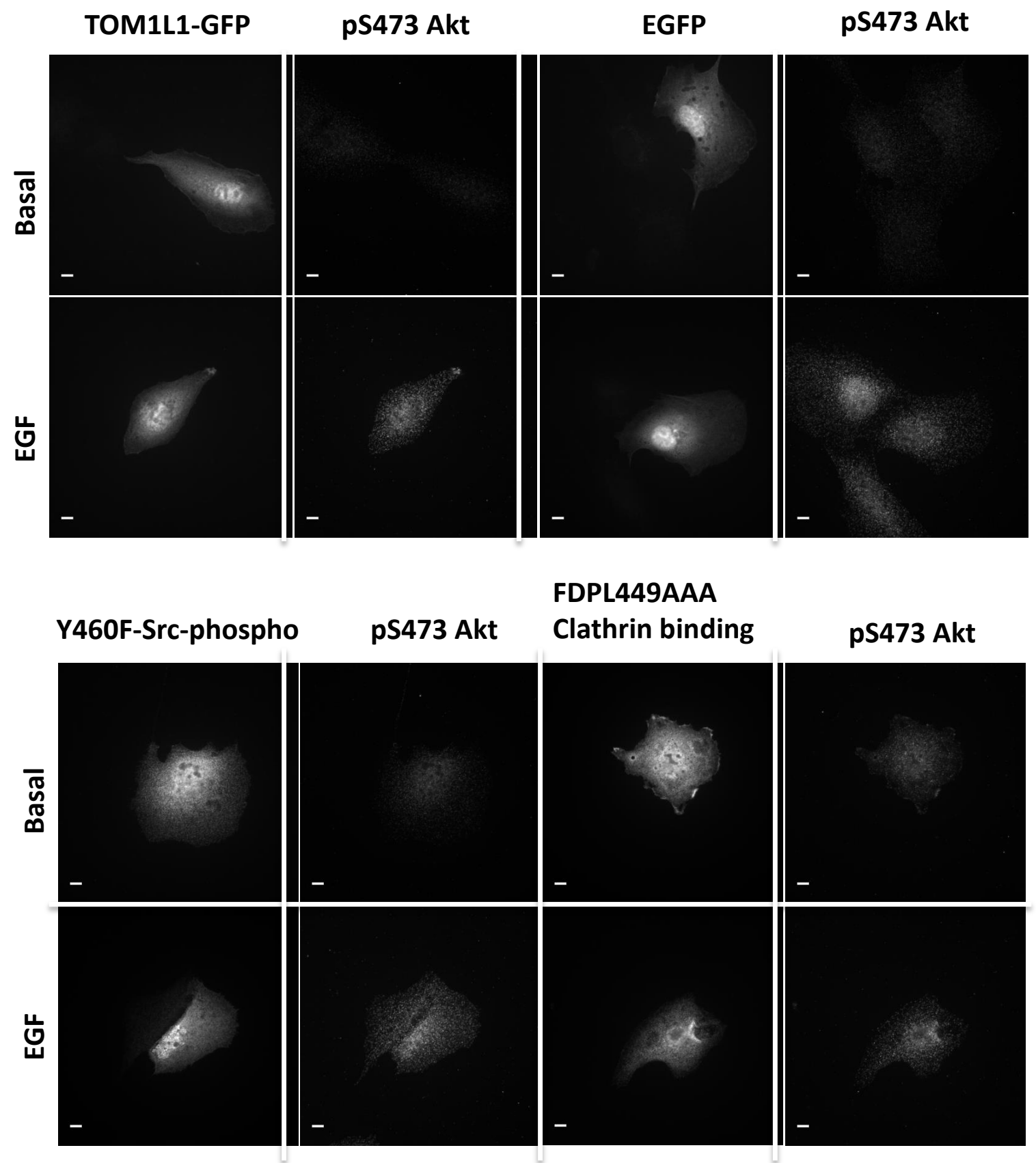
B

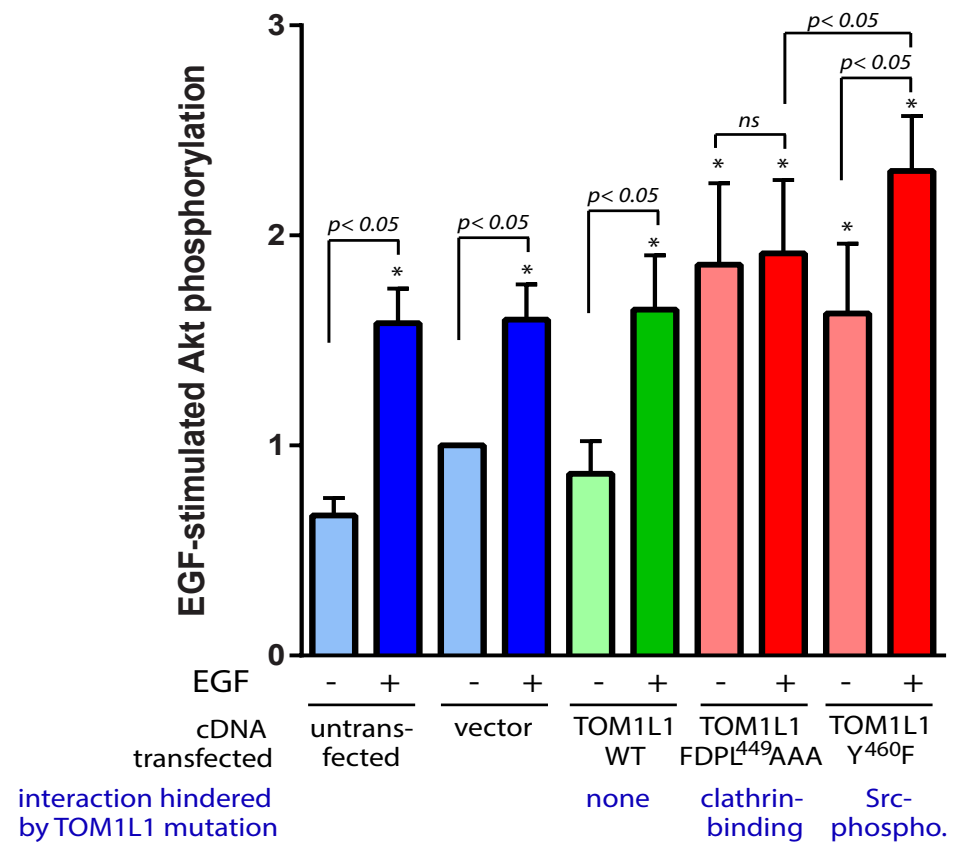

$\mathrm{C}$

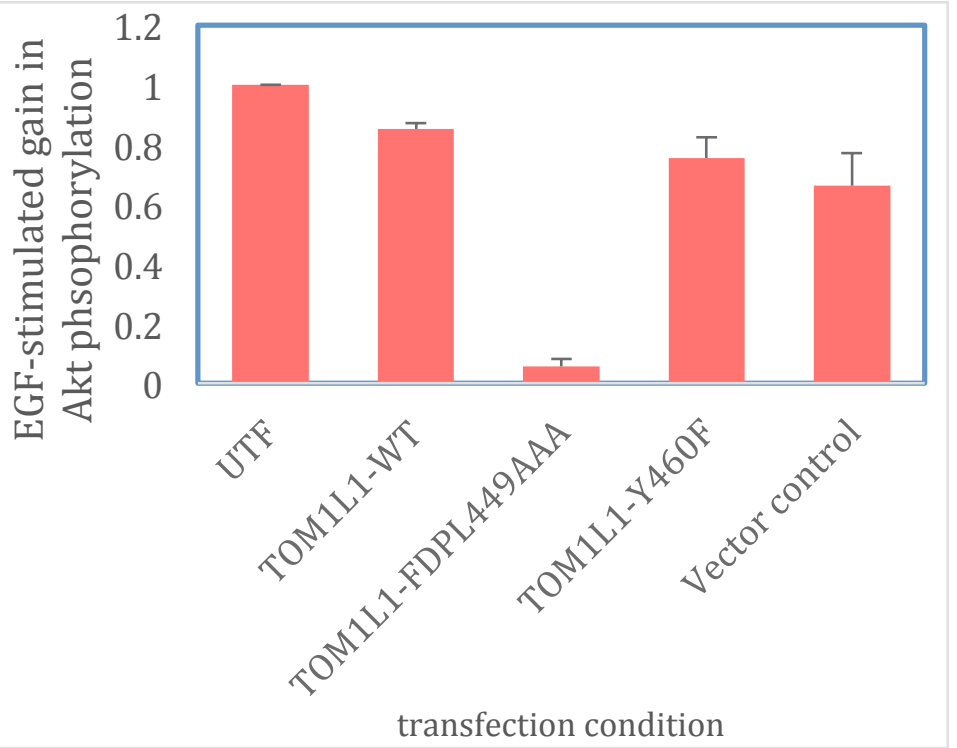

Figure 19: Clathrin binding mutant of TOM1L1 inhibits gain in EGF stimulated Akt phosphorylation. RPE-wt cells stably were transfected with TOM1L1-GFP, enhanced-GFP (EGFP), Src phosphorylation mutant (Y460F), Clathrin binding mutant (FDPL449AAA) or left untransfected (UTF). Cells were then stimulated with $5 \mathrm{ng} / \mathrm{mL}$ EGF for $5 \mathrm{~min}$ or left unstimulated (basal). Following this, cells were fixed and processed for immunofluorescence staining using anti-phospho-Akt (pS473) specific antibodies. (A) Shown are representative micrographs, scale bar $=20 \mu \mathrm{m}$. (B) The mean fluorescence intensity of phosphoAkt (pS473) staining was quantified in cells expressing TOM1L1-GFP, EGFP, FDPL449AAA, Y460F plasmids and UTF, shown are the mean \pm SE of the EGF-stimulated gain in phosphoAkt (pS473) levels (n 
$=3) .{ }^{*}, \mathrm{p}<0.05 .(\mathrm{C})$ The difference in mean fluorescence intensity (delta- $\Delta$ ) of phospho-Akt (pS473) staining for basal and stimulated conditions was quantified in cells expressing the plasmids (TOM1L1GFP, EGFP, FDPL449AAA, Y460F and UTF). Shown are the mean- $\Delta \pm$ SE of the EGF-stimulated gain in phosphoAkt (pS473) levels $(\mathrm{n}=3), \mathrm{p}<0.05$.

TOM1L1 is required for EGF stimulated Akt phosphorylation, evident from impaired Akt activation upon TOM1L1 knockdown. This function of TOM1L1 is clathrin dependent as it is constitutively recruited to CCPs and expression of TOM1L1FDPL449AAA inhibits gain in EGF stimulated Akt phosphorylation. 


\section{Discussion:}

In addition to siRNA gene silencing and small moleclule inhibition of clathrin, knocksideways silencing of clathrin also results in impaired EGF stimulated Akt phosphorylation. Furthermore, clathrin acts as a scaffold recruiting activated (pGab1) to CCPs upon EGF stimulation. TOM1L1 is constitutively recruited to CCPs and resulted in a decrease in EGF stimulated Akt phosphorylation upon TOM1L1 knockdown. Finally expression of a clathrin binding mutant of TOM1L1 in RPE-wt cells acted as dominant negative and inhibited the gain in Akt phosphorylation following EGF stimulation. Collectively the results indicate a role for TOM1L1 in regulating EGFR signaling at the plasma membrane based on its interaction with clathrin.

\section{Defining the role of Clathrin in EGF stimulated Akt phosphorylation using knocksideways silencing.}

siRNA gene silencing of $\mathrm{CHC}$ by two different sequences and clathrin inhibition by pitstop2 both result in impaired EGF stimulated Akt phosphorylation (Garay et al., 2015). In addition to these approaches, rerouting of CHC alongside with CLC to the mitochondria in the presence of rapamycin also causes a reduction in Akt phosphorylation following EGF stimulation. Protein localization is one of the key events that help regulate cell functions. For example, many proteins translocate to the nucleus or to the plasma membrane from the cytosol upon activation and control various cellular functions. In the context of this research the interaction between Akt and PDK1 usually 
requires translocation to the plasma membrane prior to Akt activation (Ding et al., 2011). Usually translocation events are accomplished within few seconds or even less time. Most other techniques for protein manipulation are based on changing protein numbers rather than localization. This includes transfection/overexpression and RNAi methods that require 10-72 $\mathrm{h}$ for showing an often-incomplete effect, or various drug treatments that often have some off target effects.

Rapamycin induced heterodimerization (aka Knocksideways) is an acute and specific method to manipulate proteins. It exploits the property of protein translocation to determine protein function. Rapamycin induced heterodimerization has been recently used to study the function of various proteins. One prominent example is recruitment of rapamycin-inducible phosphatidylinositol 4,5-bisphosphate $\left[\operatorname{PtdIns}(4,5) \mathrm{P}_{2}\right]$ 5-phosphatase to the plasma membrane. The recruitment of this phosphatase evoked rapid depletion of phosphatidylinositol 4,5-bisphosphate $(\operatorname{PtdIns}(4,5) \mathrm{P} 2)$ levels from the plasma membrane (Balla et al., 2006; Meyer et al., 2006). Since then this system has been used to study the role of PtdIns $(4,5) \mathrm{P}_{2}$ on diverse cellular processes. For example, this strategy has been used to demonstrate that the complete ablation of PIP2 from the PM results in near complete ablation of clathrin-coated pits from the cell surface (Zoncu et al., 2007). This illustrates the use of rapamycin-induced heterodimerization to cause selective recruitment of a particular protein or enzyme to a particular cellular locale to directly and acutely study the role of this protein in that specific locale. In contrast, knocksideways silencing uses a similar rapamycin-induced heterodimerization strategy to recruit specific proteins away from their normal cellular locales (ie to the surface of mitochondria). This illustrates the broad range of uses for this experimental toolbox. 
Rapamycin is a known inhibitor of mTOR and the use of rapamycin has greatly facilitated the understanding of its biological functions. mTOR is part of two major signalling complexes mTORC1 and mTORC2 (Ballou \& Lin, 2008). mTORC1 regulates various cellular functions including translation, ribosome biogenesis, autophagy, glucose metabolism, and the cellular response to hypoxia and is sensitive to rapamycin (Ballou \& Lin, 2008). On the other hand, mTORC2 phosphorylates the protein kinase Akt at Ser473 (Ballou \& Lin, 2008). More importantly, mTORC2 is insensitive to rapamycin (Ballou \& Lin, 2008). Hence, the decrease in phosphorylation of Akt in (Figure 11) is not due to the inhibition of mTOR but indeed is due to knocksideways silencing of clathrin. However, another study indicated that rapamycin does indeed render mTORC2 sensitive upon prolonged exposure (Sabatini et al., 2006). Treatment of Hela and PC3 cells with rapamycin for 24 hours inhibited mTORC2, however did not affect mTORC2 activity even when treated for 30 minutes (Sabatini et al., 2006). mTORC2 activity was assayed by its dissociation from the rictor complex and the phosphorylation of Akt at S473. While performing knocksideways silencing cells were treated with rapamycin for only 10 minutes, indicating that the impairment in EGF stimulated Akt phosphorylation was indeed due to clathrin perturbation and not mTORC2 inhibition.

The interaction between rapamycin and the FKBP domain, and between the FKBP-rapamycin complex and the FRB domain both have a $\mathrm{K}_{\mathrm{d}}<1 \mathrm{nM}$ (Crabtree et al., 2006), thus indicating a high affinity interaction. The recruitment of certain adaptors to the membrane occurs with low to moderate affinity with $\mathrm{k}_{\mathrm{d}}$ in the micromolar range (Evans et al., 2004). In addition, the FKBP-rapamycin-FRB interaction has a very slow off rate: dissociation of rapamycin from the FKBP domain has been reported to have a 
t1/2 of $17.5 \mathrm{hr}$ (Houghton et al., 1999). Also the mitochondria are present throughout the cell; thereby sequestering most of the clathrin to the mitochondrial surface upon addition of rapamycin.

Using knocksideways silencing I further established the role of clathrin as a signaling scaffold independent of endocytosis. CCPs act as signaling platforms that recruit several signaling adaptors during the residence of activated EGFR within them.

\section{CCPs as signaling microdomains at the plasma membrane.}

Upon ligand binding EGFR is recruited to CCPs where it resides until internalized into cells via formation of clathrin-coated vesicles. Clathrin assemble into a lattice on the inner leaflet of the plasma membrane and recruits other proteins and lipids, which together facilitate the internalization of these clathrin vesicles. The process of formation and internalization can take between 20 s to several minutes. Importantly, not every CCP formed at the plasma membrane is destined to be internalized; most of the CCPs formed $(>60 \%)$ are abortive, meaning that clathrin and other proteins disassemble from these structures without yielding an internalized vesicle. These abortive pits could potentially be special microdomains facilitating signalling and not just failed endocytosis events. CCPs could potentially regulate EGFR signalling leading to Akt phosphorylation. Indeed, pGab1 is recruited to CCPs upon EGF stimulation (Figure 13).

Gab1 is actively recruited to the plasma membrane upon activation of EGFR via interaction with EGFR bound Grb2. Following this Gab1 is activated by phosphorylation of Y447, Y472 and Y589. These pYs on Gabl serve as docking sites for PI3K binding 
and activation. Other pYs including Y627 recruit protein tyrosine phosphatases such as SHP-2. Gab1 pY627 acts as a docking and activation site for the tyrosine phosphatase SHP-2, a protein that has been reported to both potentiate and limit PI3K and Akt activation (Wu et al., 2001), thus further complicating the role of clathrin in regulating Gab1 phosphorylation leading to PI3K activation. That activated EGFR (bound to Grb2) resides with CCPs (Fortian and Sorkin, J Cell Sci. 2014), suggests clathrin may be required for phosphorylation of multiple residues on Gab1, thus controlling EGF stimulated Akt activation.

In order to determine the enrichment of pGab1 in CCPs, I employed TIRF microscopy (TIRF-M). By using TIRF-M, I was able to selectively image the most membrane proximal $\sim 65 \mathrm{~nm}$ of the ventral side of a cell, thus successfully eliminating any fluorescence that may be emitted by endosomes, other cellular structures or from molecules diffusing through the cytoplasm. To accomplish this, I used a RPE-RFP cell line that stably expresses RFP-clathrin light chain; these cells have been well characterized to have normal assembly of clathrin (Aguet et al., 2013). TIRF-M functions by generating an evanescent wave that propagates in the $\mathrm{z}$-direction (axial to the coverslip) and gradually degrades, which arises from the glass/water/specimen interface. This allows us to image the most proximal 200nm to the cell surface, this depth is called the penetration depth and can be fixed anywhere from $60-200 \mathrm{~nm}$ by adjusting the angle of incidence at which the laser light meets the coverslip. Using this technique, RPE-RFP cells stimulated with $5 \mathrm{ng} / \mathrm{ml}$ of EGF exhibited enrichment of pGab1 in clathrin structures at the plasma membrane. Using automated image analysis as in (Aguet et al., 2013), pGab1 resulted in a 2.3 fold increase in enrichment within CCPs. Indeed I also found that 
pGab1 enrichment is largely restricted to clathrin structures that also contain EGFR (Figure 14). A proportion of pGab1 immunofluorescence signal was found outside of CCPs in both basal and EGF stimulated cells. While for basal cells this signal most likely is a result of non-specific background staining as little signal was detected in this condition by western blotting (Figure 3). As for the EGF stimulated condition, the signal outside of CCPs could have resulted from Gab1 binding to PIP3 via its PH domain (Rodrigues et al., 2000). Gab1 may first be recruited to clathrin structures, allowing subsequent binding of pGab1 to PIP3 throughout the plasma membrane. Since Gab1 recruits both positive and negative regulators of upon activation, clathrin can be involved in both aspects of EGFR signalling leading to Akt phosphorylation. Evident by the fact that pGab1 is recruited to clathrin structures upon EGF stimulation (Figure 13), and Gab1 fails to completely activate in the absence of clathrin (Figure 3).

Clathrin may serve as a signalling scaffold by recruiting signalling adaptors upon EGFR residence in CCPs, as seen for Gab1 and other adaptor proteins mentioned above. Clathrin seems to be critical in facilitating complete activation of Gab1, but the mechanism surrounding this event remains unclear due lack of evidence of a direct interaction between Gab1 and clathrin. Recruitment of other signalling adaptors and nonreceptor tyrosine kinases to CCPs, including TOM1L1, may facilitate EGF stimulated Akt activation.

Clathrin recruits several other proteins to the plasma membrane, which are involved in membrane remodelling, facilitating the process of vesicle formation or can be 
signalling adaptors that promote signal transduction. Some of the proteins that are recruited via interaction with clathrin and AP-2 and function to promote vesicle formation are as follows:

Epsin binds to the alpha and beta-2 ear domain of the AP-2 complex (Schmid et al., 2006). Epsin belongs to a family of proteins that are important for promoting membrane curvature. Epsin consists of a N-terminus Epsin N-Terminal Homolog (ENTH) domain that binds membrane lipid phosphatidylinositol-4,5-bisphosphate (PtdIns $\left.(4,5) \mathrm{P}_{2}\right)$ and modifies membrane curvature upon binding (Ford et al., 2002). The C-terminal domain of epsin harbors several specific sequence motifs that bind to clathrin and AP-2 (Drake et al., 2000). Epsin also contains a few ubiquitin-interaction motifs (UIMs) that interact with polyubiquitins and may capture ubiquitinated cargo receptors for internalization (Polo et al., 2002).

Synaptojanin-1 is a 5'-inositol phosphatase, that localizes to CCPs and controls early stabilization and maturation efficiency (Antonescu et al., 2011). It regulates levels of membrane PtdIns(4, 5)P2 (Lee et al., 2004). The synaptojanin 1 molecule comprises two distinct inositol phosphatase domains and a $\mathrm{COOH}$-terminal proline-rich domain (PRD) (Lee et al., 2004). The central phosphatase domain dephosphorylates PI (4,5)P2 (and also PI 3,4,5-trisphosphate) selectively at the 5 position (5-phosphatase domain), whereas the NH2-terminal Sac1 domain can act on several positions of the inositol ring (Leet et al., 2004). It also contains a C-terminal SH-3 domain that interacts with several proteins including endophilin-1 (Lee et al., 2004). Two splice variants exist for synaptojanin-1 
both of which are recruited to CCPs via interaction with clathrin and AP-2 (Perera et al., 2006).

Endophilin is thought to be a particularly important interactor of synaptojain-1 and to play a major role in its recruitment to sites of endocytosis (Perera et al., 2006). Endophilin binds synaptojanin by its C-terminal SH3 domain and also contains an Nterminal BAR domain (Itoh \& Camilli, 2006). The BAR domain has been implicated to have curvature generating and sensing properties and may facilitate the maturation and generation of endocytic bud necks (Itoh \& Camilli, 2006).

CALM (clathrin assembly lymphoid myeloid leukemia protein) is another abundant clathrin adaptor that is recruited to clathrin coated vesicles (Miller et al., 2015). CALM and its neuronal specific homolog AP180, possess large C-terminal tails containing clathrin, AP2 alpha and beta2 appendage binding sites (Ford et al., 200), and a membrane-proximal, PtdIns(4,5)P2-binding N-terminal ANTH stacked-helical domain (Ford et al., 2015). CHC is a major binding protein on the C-terminal of CALM. CALM has also been implicated in controlling the size of calthrin-coated vesicles, as it also has a N-terminal membrane curvature sensing/generating amphipathic helix (Miller et al., 2015).

In addition to proteins involved in CCPs stabilization and formation afore mentioned, several signaling adaptor proteins are also recruited to CCPs including:

CIN85 (Cbl interacting protein-85 $\mathrm{kDa}$ ) belongs to a family of ubiquitously expressed adaptor molecules containing three SH3 domains, a proline-rich region and a coiled-coil 
domain (Dikic, 2002). A number of studies have suggested that CIN85 participates in both the initial steps of EGFR internalization (Soubeyran et al., 2002) and also in receptor trafficking and degradation (Kowanetz et al., 2004). CIN85 also associates with accessory endocytic proteins including AP-2 (via alpha ear), components of the actin cytoskeleton as well as other adaptor proteins involved in RTK signalling. Importantly CIN85 binds the p85 subunit of PI3K and negatively regulates its activity (Dikic, 2002).

It has also been reported to bind Src, Fyn, Yes and might play a role in regulating kinase activity (Kirsch et al., 1999). Of the most characterized role CIN85 binds Cbl and regulates Cbl-dependent EGFR ubiquitinylation and has been implicated in ubiquitin dependent EGFR degradation (Kowanetz et al., 2004).

Furthermore a non-receptor tyrosine kinase $A c k 1$ forms direct contacts with clathrin and is concentrated in CCPs when stimulated with EGF under conditions in which cells lack dynamin, thus isolating CCP-EGFR signalling (Shen et al., 2011). Ack1 has also been shown to bind Akt and phosphorylate Akt on (Y176) (Mahajan et al., 2010).

\section{TOM1L1 and Clathrin: Regulation of EGFR signalling.}

Clathrin structures at the plasma membrane serve as signalling microdomains, as they have been shown to regulate G-protein signaling by sequestering $\mathrm{G} \alpha$-interacting protein (GAIP) under unstimulated conditions, as well as serve as a scaffold regulating G-protein signaling sequestering $\delta$-opioid receptor, Gai3, and GAIPs under stimulated 
conditions (Elenko et al., 2003). Therefore, we hypothesize that a similar role for clathrin is necessary in EGF-stimulated phosphorylation of Akt. As clathrin has no catalytic properties itself, the requirement for clathrin as a microdomain must rely on its ability to recruit a number of proteins including kinases. Specifically, we are interested in TOM1L1, which forms a direct interaction with clathrin, is tyrosine phosphorylated by SFKs, and forms a transient interaction with EGFR mediated by Grb2/Shc necessary for endocytosis of the receptor upon EGF stimulation (Liu et al., 2009). EGF-stimulated Akt phosphorylation has been shown to be impaired by the Src-family kinases (SFKs) inhibitor PP1 (Kassenbrock et al., 2002), inhibition of clathrin by pitstop, siRNA knock down and knocksideways silencing also impairs EGF stimulated Akt phosphorylation. These observations suggest a role of clathrin in scaffolding SFKs or other kinases to facilitate EGF-stimulated Gab1 phosphorylation.

Clathrin interacts with TOM1L1, which in turn binds and activates SFKs, suggesting a role for TOM1L1 in early EGFR signaling. Indeed, siRNA gene silencing of TOM1L1 impairs EGF stimulated Akt phosphorylation (Figure 15). The effect of TOM1L1 knockdown is specific to EGF stimulated Akt phosphorylation, evident from the fact the TOM1L1 knockdown was of no affect on EGF stimulated EGFR phosphorylation (pY1068) (Figure 16) also normal MAPK p42/44 activation was observed (Figure 16).

Next I examined the recruitment on TOM1L1 to CCPs using TIRF-M. TOM1L1 has been documented to bind clathrin, however its recruitment to the plasma membrane CCPs has never been demonstrated. Using TIRF-M I demonstrated that TOM1L1 is constitutively recruited to plasma membrane CCPs (Figure 18). Using automated analysis 
as in (Aguet et al., 2013), I demonstrated that TOM1L1 is enriched in CCPs in both basal and EGF stimulated conditions. However, a decrease in TOM1L1 enrichment in CCPs was observed in EGF stimulated conditions as compared to basal (Figure 18B). TOM1L1 has been shown to regulate EGF stimulated endocytosis of EGFR (Liu et al., 2009). The loss in TOM1L1 enrichment in CCPs upon EGF stimulation can be attributed to Tom1L1 colocalization with EGFR in endocytic vesicles and then the sorting endosome (Liu et al., 2009). TOM1L1 is transiently Tyr-phosphorylated upon EGF stimulation, which likely facilitates its interaction with EGFR. Therefore, the role of Tom1L1 in facilitating the regulated endocytosis of EGFR might depend on its ability to interact with both activated EGFR and clathrin (Liu et al., 2009). Interestingly association of Tom1L1 with endosomes persists even when Tom1L1 is no longer Tyr-phosphorylated (Puertollano, 2005), suggesting that once in the sorting endosome, Tom1L1 may engage in other interactions for membrane binding, which is different from the Grb2/Shc-mediated interaction with activated EGFR during endocytosis. This interaction might be mediated by TOM1L1's association with TSG101 and/or Hrs (Puertollano, 2005). Thus the decrease in EGF stimulated enrichment of TOM1L1 in CCPs can be attributed to its recruitment into endosomes upon prolonged EGF stimulation. It is also possible that TOM1L1 recruitment to CCPs is required for the subsequent localization of TOM1L1 to endosomes; however this has not yet been explored.

Finally the effect on EGF stimulated Akt phosphorylation was assayed in single cells upon expression of clathrin binding (FDPL449AAA) and Src phosphorylation (Y460F) mutants of TOM1L1 (Figure 19). The clathrin-binding mutant of TOM1L1 resulted in an increase in EGF-stimulated Akt phosphorylation (Figure 19B). However, a 
significant gain in Akt phosphorylation was also observed in the basal conditions for both TOM1L1-FDP449AAA and TOM1L1-Y460F expressing cells (Figure 19B). One can attribute the failure of gain in EGF-stimulated Akt phosphorylation to having achieved the maximal saturation of Akt phosphorylation in a given cell in the basal state, given the high level of Akt phosphorylation in cells expressing TOM1L1-FDP449AAA or TOM1L1-Y460F. However, this was not the case, as evident from the increase in Akt phosphorylation in TOM1L1-Y460F expressing cells upon EGF-stimulation. Importantly, cells expressing TOM1L1-Y460F had similar levels of Akt phosphorylated in the basal state as compared to the TOM1L1-FDPL449AAA expressing cells (Figure 19B). Thus, the loss in EGF stimulated Akt phosphorylation in the TOM1L1FDPL449AAA expressing cells was a result of Akt inhibition and not simply due to an increase in Akt phosphorylation in the basal state.

These results suggest that the interaction of TOM1L1 with clathrin and Srcdependent phosphorylation of TOM1L1 negatively regulates Akt phosphorylation in the basal state (i.e. in the absence of EGF stimulation), and that the interaction of clathrin and TOM111 is required for the EGF-stimulated gain in Akt phosphorylation. Since similar basal Akt activation profiles were observed for cells expressing TOM1L1-FDPL449AAA and TOM1L1-Y460F suggests that Src-dependent phosphorylation of TOM1L1 may be clathrin dependent. I will discuss the two phenotypes observed as follows: (i) the increase in basal (i.e. in the absence of EGF stimulation) phosphorylation of Akt upon expression of TOM1L1-FDPL449AAA and TOM1L1-Y460F and (ii) the loss of EGF-stimulated Akt phosphorylation upon expression of TOM1L1-FDPL449AAA, as follows:

(i) TOM1L1 negatively regulates the mitogenic activity of Src initiated by 
platelet-derived growth factor (PDGF) (Franco et al., 2006). Src mitogenic signaling induced by PDGF is initiated in cholesterol-enriched microdomain caveolae (Collin et al., 2007). Tom1L1, through its interaction with $\mathrm{CHC}$ forms Tom1L1-CHC complex, and this interaction reduces the incorporation of Src kinases into the caveolae, thereby preventing its association with the PDGF receptor that is required for the induction of mitogenesis. The Tom1L1-CHC complex is also able to reduce the level of oncogenic Src in cholesterol-enriched microdomains (Franco et al., 2006; Collin et al., 2007). Lipid microdomains termed lipid rafts may also act as signaling platforms for EGFR signaling (Simons\&Toomre, 2000; Maxfeild, 2002; Mineo, 1999), and lipid rafts partly overlap with CCPs (Puri et al., 2005). Thus the impairment of EGF stimulated Akt phosphorylation upon defective clathrin binding/ clathrin knockdown and increase in EGF stimulated Akt phosphorylation in SPM expressing cells suggest both a positive and negative regulation of EGFR signaling by TOM1L1.

(ii) Tom1L1 interacts with Grb2 as well as the p85 subunit of PI3K in an SFK-dependent manner (Seykora et al., 2002; Li et al., 2005). The p85 subunit of PI3K also binds activated Gab1 on multiple pY-phosphorylated residues (Gu \& Neel, 2003). Interestingly, pGab1 is enriched within CCPs upon EGF stimulation, suggesting that clathrin might function as a scaffold to recruit all these signaling adaptor proteins eventually leading to 
TOM1L1 phosphorylation, which may occur in a Src-dependent manner. Therefore, we see an inhibition in gain in Akt phosphorylation upon EGF stimulation in cells expressing a TOM1L1 mutant defective in interaction with clathrin.

Taken together, these results suggest a novel role of TOM1L1 and clathrin in regulating early EGFR signaling. Signaling pathways are complex and involve various signaling regulators and adaptor proteins. Some signaling proteins interact with one another based on multiple, low affinity interactions as opposed to having single high affinity interactions (Pawson, 1997). This type of interactions results in signal amplification as the adaptor proteins can be redistributed to different cellular locales where they further activate other proteins (Pawson, 1997). TOM1L1 satisfies this requirement of being a signalling adaptor as it transiently binds various other proteins. Establishing the role of TOM1L1 in EGFR signalling future questions can be directed to explore the role of TOM1L1 in EGFR signaling in Src-overexpressing cancer cells, as Tom1L1-CHC complex is also able to reduce the level of oncogenic Src in cholesterolenriched microdomains (Franco et al., 2006; Collin et al., 2007). Different types of tumours exhibit dramatic overexpression of EGFR, including breast, brain, head and neck, gastric, pancreatic, ovarian, lung, esophageal and colorectal cancers (Salomon et al., 1995). Despite the importance of increased EGFR expression in cancer, little is known about how EGFR signalling and membrane traffic relate to the expression level of the receptor. Src has also been shown to facilitate proliferation, angiogenesis, differentiation, migration, invasion, and regulation of apoptosis in colorectal cancer cells 
(Kopetz, 2007). Src is also involved in the EGFR signal transduction pathway (Kopetz et al., 2007). Overexpression of Src has been shown to increase DNA synthesis in response to EGF (Twamley-Stein et al., 1993). On the other hand, EGF-induced DNA synthesis can be inhibited by the overexpression of an inactivated form of Src (Roche et al., 1995). Furthermore, overexpression of Src and EGFR led to increased EGF-dependent tumour formation in nude (inhibited immune system) mice (Maa et al., 1995). Taken together, these observations suggest that EGFR and Src may potentiate receptor-mediated tumorigenesis.

Interestingly, reduced levels of TOM1L1 have also been reported in human cutaneous neoplasia (Meulener et al., 2009), implying a negative role of TOM1L1 in cell proliferation. Therefore, it will be interesting to determine as to how TOM1L1 impacts cancer cell growth. The requirement of TOM1L1 in early EGFR signaling leading to Akt phosphorylation suggests a possible role for TOM1L1-Clathrin complex in EGFR cancer signaling. Formation of EGFR-ErbB2 heterodimers relieves the direct requirement for clathrin in EGF-stimulated Akt phosphorylation (Garay et al., 2015, In press). ErbB2 is unique among members of the ErbB2 tyrosine kinases (including EGFR) in its ability to bind and activate c-Src directly (Marcotte et al., 2009). TOM1L1 binds and activates Src (Liu et al., 2009). As SKFs may directly phosphorylate Gab1 (Guu \& Neel, 2003), EGFR-ErbB2 signaling may occur independent of TOM1L1. In contrast, TOM1L1 might be required for activation of SFKs during EGFR signalling, facilitated by clathrin scaffolding. In addition to EGFR, other RTKs including Insulin receptor (IR) and Met/Hepatocyte growth factor receptor (HGFR) are also recruited to CCPs upon 
activation (Goh \& Sorkin 2013). Interestingly both the receptors activate Gab1 upon ligand binding (Weidner et al., 1996; Rocchi et al., 1998), hence, it is possible that clathrin serves as a scaffold to recruit other non-receptor tyrosine kinases including TOM1L1 and facilitate their signalling. Thus, its possible that TOM1L1-Clathrin complex regulates signalling of various different RTKs. 


\section{References:}

Aguet, F., Antonescu, C. N., Mettlen, M., Schmid, S. L., and Danuser, G. (2013). Advances in Analysis of Low Signal-to-Noise Images Link Dynamin and AP2 to the Functions of an Endocytic Checkpoint. Dev. Cell 26, 279-291.

Amandio V. Vieira, Christophe Lamaze, and Sandra L. Schmid, Science 20 December 1996: 274 (5295), 2086-2089. [DOI:10.1126/science.274.5295.2086]

Antonescu, C. N., Aguet, F., Danuser, G. \& Schmid, S. L. Phosphatidylinositol-(4,5)bisphosphate regulates clathrin-coated pit initiation, stabilization, and size. Molecular biology of the cell 22, 2588-600 (2011).

Antonescu, C. N., Danuser, G. \& Schmid, S. L. Phosphatidic acid plays a regulatory role in clathrin-mediated endocytosis. Molecular Biology of the Cell 21, 2944-2952 (2010).

Antonescu CN, et al. 2008. Clathrin-dependent and independent endocytosis of glucose transporter 4 (GLUT4) in myoblasts: regulation by mitochondrial uncoupling. Traffic. 9: 1173-1190.

Ballou, Lisa M., and Richard Z. Lin. "Rapamycin and mTOR Kinase Inhibitors."Journal of Chemical Biology 1.1-4 (2008): 27-36. PMC.

Bayle J.H., Grimley J.S., Stankunas K., Gestwicki J.E., Wandless T.J., Crabtree G.R. Rapamycin analogs with differential binding specificity permit orthogonal control of protein activity. Chem. Biol. 2006;13:99-107.

B.C. Suh, T. Inoue, T. Meyer, B. Hille. Rapid chemically induced changes of PtdIns(4,5)P2 gate KCNQ ion channels. Science, 314 (2006), pp. 1454-1457

Bonifacino JS (2004) The GGA proteins: adaptors on the move. Nat Rev Mol Cell Biol 5: 23-32

Bromann, P. A., H. Korkaya, and S. A. Courtneidge. 2004. The interplay between Src family kinases and receptor tyrosine kinases.Oncogene 23: 7957-7968.

Burgering, B. M. T. (2003). Decisions on life and death : FOXO Forkhead transcription factors are in command when PKB / Akt is off duty, 73(June), 689-701.

doi:10.1189/jlb.1202629.Journal

Chan, P.-C. et al. Src Phosphorylates Grb2-associated Binder 1 upon Hepatocyte Growth Factor Stimulation. Journal of Biological Chemistry 278, 44075-44082 (2003).

Collin G, Franco M, Simon V, Benistant C, Roche S.The Tom1L1-clathrin heavy chain complex regulates membrane partitioning of the tyrosine kinase Src required for mitogenic and transforming activities.Mol Cell Biol2007;27:7631-7640. 
Conner SD, Schmid SL. 2003. Regulated portals of entry into the cell. Nature 422:37-44

Cross, D., Alessi, D., Cohen, P., Andjelkovich, M., \& Hemmings, B. (1995). Inhibition of glycogen synthase kinase-3 by insulin mediated by protein kinase B.

Das, M. et al. Regulation of Neuron Survival through an Intersectin-Phosphoinositide 3'Kinase C2ß-AKT Pathway. Molecular and Cellular Biology 27, 7906-7917 (2007).

Dikic I. CIN85/CMS family of adaptor molecules. FEBS Lett. 2002 Oct 2;529(1):110-5. Review.

Downward J, Parker P, Waterfield MD (1984). "Autophosphorylation sites on the epidermal growth factor receptor". Nature 311 (5985): 483-5. doi:10.1038/311483a0. PMID 6090945.

Drake MT, Downs MA, Traub LM (2000) Epsin binds to clathrin by associating directly with the clathrin-terminal domain. Evidence for cooperative binding through two discrete sites. J Biol Chem275:6479-6489.

Dutta D, Williamson CD, Cole NB, Donaldson JG (2012) Pitstop 2 Is a Potent Inhibitor of Clathrin-Independent Endocytosis. PLoS ONE 7(9): e45799

Elenko, E., Fischer, T., Niesman, I., Harding, T. I. M., Mcquistan, T., Zastrow, M. V. O. N., ... San, C. (2003). Spatial Regulation of $G_{\sqcup}$ i Protein Signaling in Clathrin-Coated Membrane Microdomains Containing GAIP, 64(1), 11-20.

Ford MG, et al. (2002) Curvature of clathrin-coated pits driven by epsin. Nature 419:361-366.

Ford M.G., Pearse B.M., Higgins M.K., Vallis Y., Owen D.J., Gibson A., Hopkins C.R., Evans P.R., McMahon H.T. Simultaneous binding of PtdIns(4,5)P2 and clathrin by AP180 in the nucleation of clathrin lattices on membranes. Science. 2001;291:10511055.

Franco M, Furstoss O, Simon V, Benistant C, Hong WJ, Roche S (2006) The adaptor protein Tom $1 \mathrm{~L} 1$ is a negative regulator of Src mitogenic signaling induced by growth factors. Mol Cell Biol 26: 1932-1947

Goh, L. K., Huang, F., Kim, W., Gygi, S. \& Sorkin, A. Multiple mechanisms collectively regulate clathrin-mediated endocytosis of the epidermal growth factor receptor. The Journal of Cell Biology 189, 871-883 (2010).

$\mathrm{Gu}$ H, Neel BG. The "Gab" in signal transduction. Trends Cell Biol. 2003 Mar;13(3):122-30. Review.

Henson E. S., Gibson S. B. (2006) Surviving cell death through epidermal growth factor signal transduction pathways: Implications for cancer therapy. Cell Sig. 
Holgado-Madruga, M., Emlet, D. R., Moscatello, D. K., Godwin, A. K. \& Wong, A. J. A Grb2-associated docking protein in EGF- and insulin-receptor signalling. , Published online: 08February 1996; | doi:10.1038/379560a0 379, 560-564 (1996).

Hosoi H., Dilling M.B., Shikata T., Liu L.N., Shu L., Ashmun R.A., Germain G.S., Abraham R.T., Houghton P.J. Rapamycin causes poorly reversible inhibition of mTOR and induces p53-independent apoptosis in human rhabdomyosarcoma cells. Cancer Res. 1999;59:886-894.

Iwamoto, R. et al. Heparin-binding EGF-like growth factor and ErbB signaling is essential for heart function. Proceedings of the National Academy of Sciences 100, 32213226 (2003).

Jaqaman, K. et al. Robust single-particle tracking in live-cell time-lapse sequences. Nature Methods 5, 695-702 (2008)

Jiang, X., Huang, F., Marusyk, A. \& Sorkin, A. Grb2 regulates internalization of EGF receptors through clathrin-coated pits. Molecular biology of the cell 14, 858-70 (2003).

Johannessen, L. E., Pedersen, N. M., Pedersen, K. W., Madshus, I. H. \& Stang, E. Activation of the epidermal growth factor (EGF) receptor induces formation of EGF receptor- and Grb2-containing clathrin-coated pits. Mol Cell Biol 26, 389-401 (2006).

Kassenbrock, C. K., Hunter, S., Garl, P., Johnson, G. L. \& Anderson, S. M. Inhibition of Src Family Kinases Blocks Epidermal Growth Factor (EGF)-induced Activation of Akt, Phosphorylation of c-Cbl, and Ubiquitination of the EGF Receptor. Journal of Biological Chemistry 277, 24967-24975 (2002).

Kirchhausen T, Owen D, Harrison SC. Molecular structure, function, and dynamics of clathrin-mediated membrane traffic. Cold Spring Harb Perspect Biol. 2014 May 1;6(5):a016725. doi: 10.1101/cshperspect.a016725. Review. PubMed PMID: 24789820.

Kirsch, K. H., Georgescu, M.-M., Ishimaru, S. \& Hanafusa, H. CMS: An adapter molecule involved in cytoskeletal rearrangements. Proceedings of the National Academy of Sciences 96, 6211-6216 (1999).

Kisseleva T., Bhattacharya S., Braunstein J., Schindler C. W. (2002) Signalling through the JAK/STAT pathway, recent advances and future challenges. Gene. 285, 1-24.

Kiyatkin E. Aksamitiene, N.I. Markevich, N.M. Borisov, J.B. Hoek, B.N. Kholodenko, Scaffolding protein Grb2-associated binder 1 sustains epidermal growth factor-induced mitogenic and survival signaling by multiple positive feedback loops. J. Biol. Chem. 281,19925-38 (2006).

Knight, J. \& Falke, J. J. Membrane Diffusion of PH Domain-PIP3 Complexes: the 
Effects of Target Lipid Stoichiometry on Diffusion Constant Probed Using SingleMolecule Fluorescence Microscopy. Biophysical Journal 98, 86a (2010).

Kopetz, Scott. "Targeting Src and Epidermal Growth Factor Receptor in Colorectal Cancer: Rationale and Progress Into the Clinic." Gastrointestinal Cancer Research : GCR 1.4 Suppl 2 (2007): S37-S41. Print.

Kowanetz K, et al. CIN85 associates with multiple effectors controlling intracellular trafficking of epidermal growth factor receptors. Mol Biol Cell. 2004;15:3155-3166.

Lee SY, Wenk MR, Kim Y, Nairn AC, De Camilli P. Regulation of synaptojanin 1 by cyclin-dependent kinase 5 at synapses. Proc Natl Acad Sci U S A. 2004 Jan 13;101(2):546-51. Epub 2004 Jan 2.

Li W, Marshall C, Mei L,Dzubow L, Schmults C,Dans M, Seykora J. Srcasm modulates EGF and Src-kinase signaling in keratinocytes. J Biol Chem2005;280: 6036-6046.

Liu, N. S., Loo, L. S., Loh, E., Seet, L.-F. \& Hong, W. Participation of Tom1L1 in EGFstimulated endocytosis of EGF receptor. The EMBO Journal 28, 3485-3499 (2009).

Lock, L. S., Royal, I., Naujokas, M. A. \& Park, M. Identification of an atypical Grb2 carboxylterminal SH3 domain binding site in Gab docking proteins reveals Grb2dependent and - independent recruitment of Gab1 to receptor tyrosine kinases. The Journal of biological chemistry 275, 31536-31545 (2000).

Lodish H, Berk A, Zipursky SL, et al. Molecular Cell Biology. 4th edition. New York: W. H. Freeman; 2000.

Loerke, D., Mettlen, M., Schmid, S. L. \& Danuser, G. Measuring the hierarchy of molecular events during clathrin-mediated endocytosis. Traffic (Copenhagen, Denmark) (2011).doi:10.1111/j.1600-0854.2011.01197.x

Loerke, D. et al. Cargo and dynamin regulate clathrin-coated pit maturation. PLoS Biol 7, e57 (2009).

Lohi O, Poussu A, Mao Y, Quiocho F, Lehto VP (2002) VHS domain —a longshoreman of vesicle lines. FEBS Lett 513: 19-23

Maa MC, Leu TH, McCarley DJ, et al. Potentiation of epidermal growth factor receptormediated oncogenesis by c-Src: implications for the etiology of multiple human cancers. Proc Natl Acad Sci U S A.1995;92:6981-6985.

Mahajan K, Coppola D, Challa S, Fang B, Chen YA, Zhu W, Lopez AS, Koomen J, Engelman RW, Rivera C, Muraoka-Cook RS, Cheng JQ, Schönbrunn E, Sebti SM, Earp 
HS, Mahajan NP. (March 2010). "Ack1 mediated AKT/PKB tyrosine 176 phosphorylation regulates its activation". PLoS ONE 5 (3): e9646.

Maxfield, F. R. Plasma membrane microdomains. Current Opinion in Cell Biology 14, 483-487 (2002).

Mattoon, D. R., Lamothe, B., Lax, I. \& Schlessinger, J. The docking protein Gab1 is the primary mediator of EGF-stimulated activation of the PI-3K/Akt cell survival pathway. BMC Biology 2, 24 (2004).

M. Holgado-Madruga, D. R. Emlet, D. K. Moscatello, A. K. Godwin, A. J. Wong, A Grb2-associated docking protein in EGF- and insulin-receptor signalling. Nature. 379, 560-564 (1996).

Matheny, R. W. \& Adamo, M. L. Current Perspectives on Akt Akt-ivation and Akt-ions. Experimental Biology and Medicine 234, 1264-1270 (2009).

Mcmanus, E. J., Alessi, D. R., \& Pkb, B. (2002). TSC1 - TSC2 : a complex tale of PKBmediated S6K regulation, 4(September), 214-217.

Meulener MC, Ayli EE,Elenitsas R, Seykora JT.Decreased Srcasm expression in hyperproliferative cutaneous lesions. J Cutan Pathol 2009;36:291-295

Mettlen, M. et al. Endocytic accessory proteins are functionally distinguished by their differential effects on the maturation of clathrin-coated pits. Molecular biology of the cell 20, 3251-60 (2009).

Mettlen, M., Loerke, D., Yarar, D., Danuser, G. \& Schmid, S. L. Cargo- and adaptorspecific mechanisms regulate clathrin-mediated endocytosis. The Journal of Cell Biology 188, 919-933 (2010).

Miettinen, P. J. et al. Epithelial immaturity and multiorgan failure in mice lacking epidermal growth factor receptor. Nature 376, 337-341 (1995).

Miller SE, Mathiasen S, Bright NA, Pierre F, Kelly BT, Kladt N, Schauss A, Merrifield CJ, Stamou D, Höning S, Owen DJ. CALM regulates clathrin-coated vesicle size and maturation by directly sensing and driving membrane curvature. Dev Cell. 2015 Apr 20;33(2):163-75.

Mineo, C., Gill, G. N. \& Anderson, R. G. W. Regulated Migration of Epidermal Growth Factor Receptor from Caveolae. Journal of Biological Chemistry 274, 30636-30643 (1999).

Motley AM, Berg N, Taylor MJ, Sahlender DA, Hirst J, et al. (2006) Functional analysis of AP-2 alpha and mu2 subunits. Mol Biol Cell 17: 5298-5308. 
Nikolaienko, O. et al. Intersectin 1 forms a complex with adaptor protein Ruk/CIN85 in vivo independently of epidermal growth factor stimulation. Cellular signalling 21, 753 759 (2009).

Nunez, D. et al. Hotspots organize clathrin-mediated endocytosis by efficient recruitment and retention of nucleating resources. Traffic (Copenhagen, Denmark) (2011).doi:10.1111/j.1600-0854.2011.01273.x

Oda K, Matsuoka Y, Funahashi A, Kitano H (2005). "A comprehensive pathway map of epidermal growth factor receptor signaling". Mol. Syst. Biol. 1 (1): 2005.0010. doi:10.1038/msb4100014. PMC 1681468. PMID 16729045.

Orton, R. J., Sturm, O. E., Vyshemirsky, V., Calder, M., Gilbert, D. R., \& Kolch, W. (2005). Computational modelling of the receptor-tyrosine-kinase-activated MAPK pathway. Biochemical Journal, 392(Pt 2), 249-261. doi:10.1042/BJ20050908

Owen D.J., Collins B.M., Evans P.R. Adaptors for clathrin coats: structure and function. Annu. Rev. Cell Dev. Biol. 2004;20:153-191.

P. Varnai, B. Thyagarajan, T. Rohacs, T. Balla. Rapidly inducible changes in phosphatidylinositol 4,5-bisphosphate levels influence multiple regulatory functions of the lipid in intact living cells. J. Cell Biol., 175 (2006), pp. 377-382

Pawson, T. (1997). "Signaling Through Scaffold, Anchoring, and Adaptor Proteins". Science 278 (5346): 2075-2080.

Perera RM, Zoncu R, Lucast L, De Camilli P, Toomre D. Two synaptojanin 1 isoforms are recruited to clathrin-coated pits at different stages. Proc Natl Acad Sci U S A. 2006 Dec 19;103(51):19332-7. Epub 2006 Dec 8

Polo S, et al. (2002) A single motif responsible for ubiquitin recognition and monoubiquitination in endocytic proteins. Nature 416:451-455.

Pritchard, J. E., Jablonski, A. B. \& Parsons, S. J. Convergence of EGF Receptor and Src FamilySignaling Networks in Cancer. 112-130 (2008). at http://www.springerlink.com/content/x414858581p18008/abstract/

Puertollano R (2005) Interactions of TOM1L1 with the multivesicular body sorting machinery. J Biol Chem 280: 9258-9264

Puri, C. et al. Relationships between EGFR Signaling-competent and Endocytosiscompetent Membrane Microdomains. Molecular Biology of the Cell 16, 2704-2718 (2005). 
Robinson, M. S., Sahlender, D. A., \& Foster, S. D. (2010). Rapid inactivation of proteins by rapamycin-induced rerouting to mitochondria. Developmental Cell, 18(2), 324-331. doi: 10.1016/j.devcel.2009.12.015

Rocchi S, Tartare-Deckert S, Murdaca J, Holgado-Madruga M, Wong AJ, Van Obberghen E. Determination of Gab1 (Grb2-associated binder-1) interaction with insulin receptor-signaling molecules. Mol Endocrinol. 1998 Jul;12(7):914-23.

Roche S, Koegl M, Barone MV, et al. DNA synthesis induced by some but not all growth factors requires Src family protein tyrosine kinases. Mol Cell Biol. 1995;15:1102-1109.

Rodrigues, G. A., Falasca, M., Zhang, Z., Ong, S. H., and Schlessinger, J. (2000). A novel positive feedback loop mediated by the docking protein Gab1 and phosphatidylinositol 3-kinase in epidermal growth factor receptor signaling. Mol. Cell. Biol. 20, 1448-1459.

Salomon, D. S., Brandt, R., Ciardiello, F. \& Normanno, N. Epidermal growth factorrelated peptides and their receptors in human malignancies. Critical Reviews in Oncology/Hematology 19, 183-232 (1995).

Sarbassov DD, Ali SM, Sengupta S, Sheen JH, Hsu PP, Bagley AF, Markhard AL, Sabatini DM. Prolonged rapamycin treatment inhibits mTORC2 assembly and Akt/PKB. Mol Cell. 2006 Apr 21;22(2):159-68. Epub 2006 Apr 6. PubMed PMID: 16603397.

Sármay, G., Angyal, A., Kertész, Á., Maus, M. \& Medgyesi, D. The multiple function of Grb2 associated binder (Gab) adaptor/scaffolding protein in immune cell signaling. Immunology Letters 104, 76-82 (2006).

Stern, D. F. ErbBs in mammary development. Experimental Cell Research 284, 89-98 (2003).

Schmid EM, McMahon HT. Integrating molecular and network biology to decode endocytosis. Nature. 2007 Aug 23;448(7156):883-8.

Schmid, E. M. et al. Role of the AP2 beta-appendage hub in recruiting partners for clathrin coated vesicle assembly. PLoS Biology 4, e262 (2006).

Schulze WX, Deng L, Mann M (2005). "Phosphotyrosine interactome of the ErbBreceptor kinase family". Molecular systems biology 1 (1): 2005.0008.

Seykora JT, Mei L, Dotto GP, Stein PL (2002) Srcasm: a novel Src activating and signaling molecule. J Biol Chem 277: 2812-2822

Shen, H., Ferguson, S. M., Dephoure, N., Park, R., Yang, Y., Volpicelli-Daley, L., ... De Camilli, P. (2011). Constitutive activated Cdc42-associated kinase (Ack) phosphorylation at arrested endocytic clathrin-coated pits of cells that lack dynamin. Molecular Biology of the Cell, 22(4), 493-502. doi:10.1091/mbc.E10-07-0637 
Sibilia, M., Steinbach, J. P., Stingl, L., Aguzzi, A. \& Wagner, E. F. A strain-independent postnatal neurodegeneration in mice lacking the EGF receptor. The EMBO journal 17, 719-731 (1998).

Sibilia, M. \& Wagner, E. F. Strain-dependent epithelial defects in mice lacking the EGF receptor. Science (New York, N.Y.) 269, 234-238 (1995).

Sigismund, S., Argenzio, E., Tosoni, D., Cavallaro, E., Polo, S., \& Di Fiore, P. P. (2008). Clathrin-mediated internalization is essential for sustained EGFR signaling but dispensable for degradation. Developmental Cell, 15(2), 209-219. doi: 10.1016/j.devcel.2008.06.012

Simons, K. \& Toomre, D. Lipid rafts and signal transduction. Nature Reviews Molecular Cell Biology 1, 31-39 (2000).

Sorkin, A. \& Goh, L. K. Endocytosis and intracellular trafficking of ErbBs. Exp Cell Res 315, 683-696 (2009).

Soubeyran P, Kowanetz K, Szymkiewicz I, Langdon WY, Dikic I. Cbl-CIN85-endophilin complex mediates ligand-induced downregulation of EGF receptors. Nature. 2002;416:183-187.

Threadgill, D. W. et al. Targeted disruption of mouse EGF receptor: effect of genetic background on mutant phenotype. Science (New York, N.Y.) 269, 230-234 (1995).

Tong, X. K., Hussain, N. K., Adams, A. G., O’Bryan, J. P. \& McPherson, P. S. Intersectin can regulate the Ras/MAP kinase pathway independent of its role in endocytosis. The Journal of biological chemistry 275, 29894-29899 (2000).

Toshiki Itoh, Pietro De Camilli, BAR, F-BAR (EFC) and ENTH/ANTH domains in the regulation of membrane-cytosol interfaces and membrane curvature, Biochimica et Biophysica Acta (BBA) - Molecular and Cell Biology of Lipids, Volume 1761, Issue 8, August 2006, Pages 897-912

Twamley-Stein GM, Pepperkok R, Ansorge W, et al. The Src family tyrosine kinases are required for platelet-derived growth factormediated signal transduction in NIH 3T3 cells. Proc Natl Acad Sci U S A.1993;90:7696-7700.

von Kleist, L., Stahlschmidt, W., Bulut, H., Gromova, K., Puchkov, D., Robertson, M. Haucke, V. (2011). Role of the clathrin terminal domain in regulating coated pit dynamics revealed by small molecule inhibition. Cell, 146(3), 471-484. doi: 10.1016/j.cell.2011.06.025 
von Kleist L, Haucke V. At the crossroads of chemistry and cell biology: inhibiting membrane traffic by small molecules. Traffic. 2012 Apr;13(4):495-504. doi:

10.1111/j.1600-0854.2011.01292.x. Epub 2011 Oct 24. Review. PubMed PMID: 21951680.

Wang, T., Liu, N. S., Seet, L.-F. \& Hong, W. The Emerging Role of VHS DomainContaining Tom1, Tom1L1 and Tom1L2 in Membrane Trafficking. Traffic 11, 11191128 (2010).

Weidner KM, Di Cesare S, Sachs M, Brinkmann V, Behrens J, Birchmeier W. Interaction between Gabl and the c-Met receptor tyrosine kinase is responsible for epithelial morphogenesis. Nature. 1996 Nov 14;384(6605):173-6.

Wong RW. Transgenic and knock-out mice for deciphering the roles of EGFR ligands. Cell Mol Life Sci. 2003 Jan;60(1):113-8. Review. PubMed PMID: 12613661.

Wu, C. J., O’Rourke, D. M., Feng, G. S., Johnson, G. R., Wang, Q., and Greene, M. I. (2001). The tyrosine phosphatase SHP-2 is required for mediating phosphatidylinositol kinase/Akt activation by growth factors. Oncogene 20, 6018-6025.

Yosef Yarden and Joseph Schlessinger (1987). "Epidermal Growth-Factor Induces Rapid, Reversible Aggregation of the Purified Epidermal Growth-Factor Receptor". Biochemistry 26 (5): 1443-1451. doi:10.1021/bi00379a035. PMID 3494473.

Z. Ding, et al. Physical association of PDK1 with AKT1 is sufficient for pathway activation independent of membrane localization and phosphatidylinositol 3 kinase PLoS ONE, 5 (2011), p. e9910

Zhou, J. \& Hsieh, J.-T. The Inhibitory Role of DOC-2/DAB2 in Growth Factor Receptormediated Signal Cascade DOC-2/DAB2-MEDIATED INHIBITION OF ERK PHOSPHORYLATION VIA BINDING TO Grb2. Journal of Biological Chemistry 276, 27793-27798 (2001).

Zoncu R, Perera RM, Sebastian R, Nakatsu F, Chen H, Balla T, Ayala G, Toomre D, De Camilli PV. Loss of endocytic clathrin-coated pits upon acute depletion of phosphatidylinositol 4,5-bisphosphate. Proc Natl Acad Sci U S A. 2007 Mar 6;104(10):3793-8. 